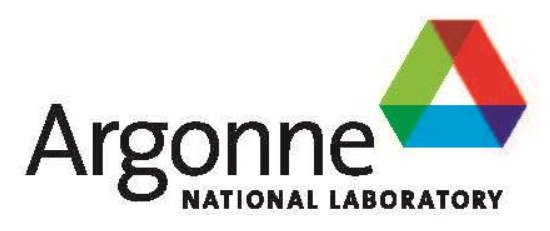

\title{
Technical Improvements to an Absorbing Supergel for Radiological Decontamination in Tropical Environments
}

Nuclear Engineering Division 


\begin{abstract}
About Argonne National Laboratory
Argonne is a U.S. Department of Energy laboratory managed by UChicago Argonne, LLC under contract DE-AC02-06CH11357. The Laboratory's main facility is outside Chicago, at 9700 South Cass Avenue, Argonne, Illinois 60439. For information about Argonne and its pioneering science and technology programs, see www.anl.gov.
\end{abstract}

\title{
DOCUMENT AVAILABILITY
}

Online Access: U.S. Department of Energy (DOE) reports produced after 1991 and a growing number of pre-1991 documents are available free via DOE's SciTech Connect (http://www.osti.gov/scitech/).

Reports not in digital format may be purchased by the public from the National Technical Information Service (NTIS):

U.S. Department of Commerce

National Technical Information Service

5301 Shawnee Rd

Alexandria, VA 22312

unw.ntis.gov

Phone: (800) 553-NTIS (6847) or (703) 605-6000

Fax: (703) 605-6900

Email: orders@ntis.gov

Reports not in digital format are available to DOE and DOE contractors from the Office of Scientific and Technical Information (OST):

U.S. Department of Energy

Office of Scientific and Technical Information

P.O. Box 62

Oak Ridge, TN 37831-0062

unw.osti.gov

Phone: (865) 576-8401

Fax: (865) 576-5728

Email: reports@osti.gov

\section{Disclaimer}

This report was prepared as an account of work sponsored by an agency of the United States Government. Neither the United States Government nor any agency thereof, nor UChicago Argonne, LLC, nor any of their employees or officers, makes any warranty, express or implied, or assumes any legal liability or responsibility for the accuracy, completeness, or usefulness of any information, apparatus, product, or process disclosed, or represents that its use would not infringe privately owned rights. Reference herein to any specific commercial product, process, or service by trade name, trademark, manufacturer, or otherwise, does not necessarily constitute or imply its endorsement, recommendation, or favoring by the United States Government or any agency thereof. The views and opinions of document authors expressed herein do not necessarily state or reflect those of the United States Government or any agency thereof. 


\section{Technical Improvements to an Absorbing Supergel for Radiological Decontamination in Tropical Environments}

by

Michael D. Kaminski, Carol J. Mertz, and Nadia Kivenas

Nuclear Engineering Division, Argonne National Laboratory

Rick Demmer

Idaho National Laboratory, Battelle Energy Alliance, LLC

August 2016 



\section{CONTENTS}

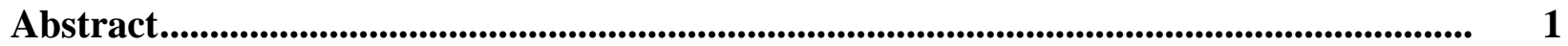

1 Background ….................................................................................................................................... 1

2 Replacement Sequestering Agents to MST and CST (Task 1) ......................................... 3

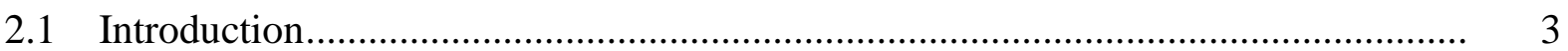

2.2 Methods .....................................................................................................

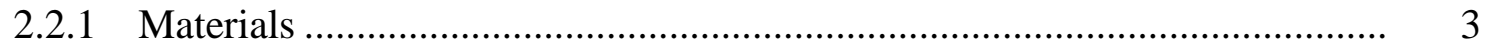

2.2.2 Determination of $\mathrm{pH}$ Dependence .............................................................. 4

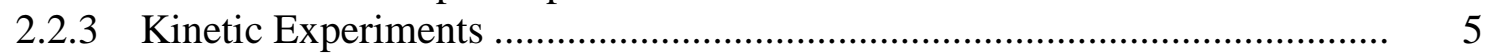

2.2.4 Sorption of Cesium from Solution............................................................... 5

2.2.5 Solid-to-Liquid Ratio ............................................................................. 5

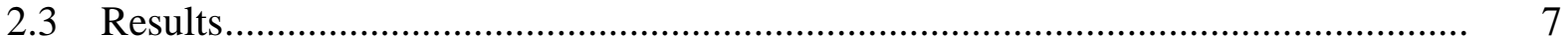

2.3.1 Properties of Candidates for Sequestering Agent Replacements................... 7

2.3.2 pH Dependence on Cesium Sequestering Agents......................................... 36

2.3.3 Kinetics of Cesium Sorption......................................................................... 36

2.3.4 Sorption of Cesium in the Presence of Salt .................................................. 38

2.3.5 Sorption of Cesium with Mixed Isotope Hydrogel Formulation .................... 38

2.3.6 Sorption of Cesium as a Function of Solid-to-Liquid Ratio ........................... 45

2.3.7 Kinetics of Americium Sorption................................................................... 47

$3 \quad$ Mixed Isotope Testing (Task 2) …................................................................................................. 50

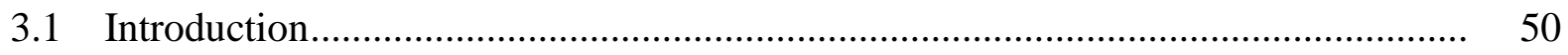

3.2 Methods ................................................................................................. 50

3.2.1 Superabsorbent Retention Capacity ....................................................... $\quad 50$

3.2.2 Concrete Coupon Testing from Mixed Isotope Gel Formulation.................. 52

3.2.3 Cesium and Americium Decontamination Using Mixed-Isotope Gel

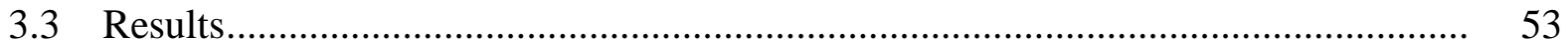

3.3.1 Retention Capacity.............................................................................. 53

3.3.2 Decontamination of Cesium from Concrete Using Mixed Isotope

3.3.3 Cesium and Americium Decontamination Using Mixed Isotope Gel Formulation................................................................................... 54

4 Effect of Variation in Concentration of Americium and Cesium Contamination (Task 3) ....................................................................................................................................... 56

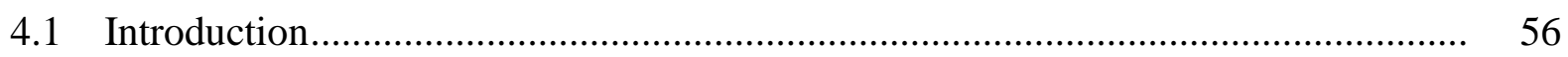

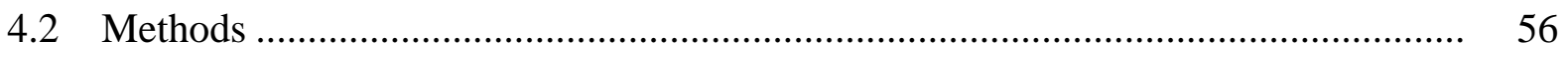

4.3 Results.............................................................................................. 58 


\section{CONTENTS (Cont.)}

$5 \quad$ Pilot-Scale Testing (Task 4)..................................................................................... $\quad 60$

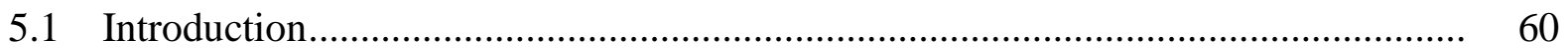

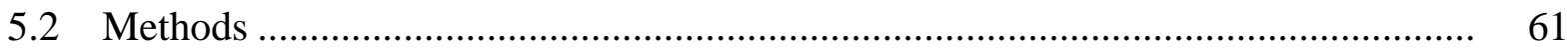

5.2.1 Idaho Testing of Combined Formulation.................................................. 61

5.2.2 Argonne Testing of Combined Formulation..................................................... 62

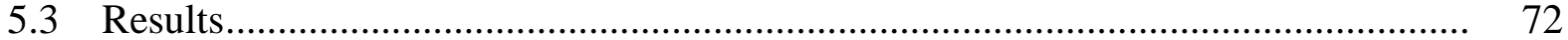

5.3.1 Density and Porosity Measurements........................................................... $\quad 72$

5.3.2 Idaho Testing of Combined Formulation....................................................... 73

5.3.2 Argonne Testing of Combined Gel Formulation............................................ 73

6 Conclusions ...................................................................................................................................... 79

Appendix A Cliniptololite Suppliers in United States .............................................................. 81

Appendix B Companies Contacted for Availability of Birnessite, Clinoptilonite, Illite, and Romanechite..................................................................................................................... 82

Appendix C Suppliers of Clinoptilolite and Chabazite ................................................................ 85

Appendix D Price for Vermiculite in Bulk .................................................................................... 87

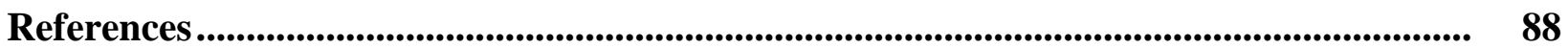

\section{FIGURES}

2-1 Volume of $1.0 \mathrm{M} \mathrm{HNO}_{3}$ needed to adjust $\mathrm{pH}$ of unbuffered clay slurry. The corresponding concentration of protons for an unbuffered solution is given in the

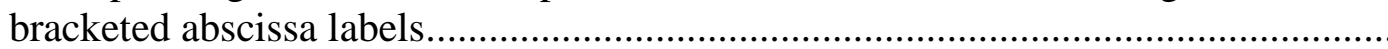

2-2 Sorption kinetics for ${ }^{137} \mathrm{Cs}$ in unbuffered water in terms of activity in the supernatant solution and percent removal of cesium from solution.

2-3 Effect of $\mathrm{KCl}$ on the equilibrium sorption of cesium onto various sequestering agents in terms of activity in the supernatant solution, partitioning coefficient $\mathrm{K}_{\mathrm{d}}$, and percent removal of cesium from solution.

2-4 Effect of $\mathrm{NH}_{4} \mathrm{Cl}$ on the equilibrium sorption of cesium onto various sequestering agents in terms of activity in the supernatant solution, partitioning coefficient $\mathrm{K}_{\mathrm{d}}$, and percent removal of cesium from solution. 


\section{FIGURES (Cont.)}

2-5 Effect of $\mathrm{Na}_{2} \mathrm{CO}_{3}$ on the equilibrium sorption of cesium onto various sequestering agents in terms of activity in the supernatant solution, partitioning coefficient $\mathrm{K}_{\mathrm{d}}$, and percent removal of cesium from solution.

2-6 Effect of HEDPA on the equilibrium sorption of cesium onto various sequestering agents in terms of activity in the supernatant solution, partitioning coefficient $\mathrm{K}_{\mathrm{d}}$, and percent removal of cesium from solution.

2-7 Sorption of Cs-137 onto clays from a solution of $1 \mathrm{M} \mathrm{K}^{+}, 0.5 \mathrm{M} \mathrm{CO}_{3}{ }^{2-}$, and $0.025 \mathrm{M}$ HEDPA after $1 \mathrm{~h}$ of gentle agitation at $10 \mathrm{mg} / \mathrm{mL}$ slurry concentration.

2-8 Sorption of Cs-137 onto chabazite from 0.0001-0.5 M solutions of $\mathrm{NH}_{4} \mathrm{Cl}, \mathrm{KCl}$, $\mathrm{Na}_{2} \mathrm{CO}_{3}$, and HEDPA or a combined solution of $1 \mathrm{M} \mathrm{K}^{+}, 0.5 \mathrm{M} \mathrm{CO}_{3}{ }^{2-}$, and $0.025 \mathrm{M}$ HEDPA. Tests were allowed to equilibrate for $1 \mathrm{~h}$ under gentle agitation at $10 \mathrm{mg} / \mathrm{mL}$ slurry concentrations.

2-9 Sorption of Cs-137 onto montmorillonite from 0.0001-0.1 M solutions of $\mathrm{NH}_{4} \mathrm{Cl}$ or a combined formulation solution of $1 \mathrm{M} \mathrm{K}^{+}, 0.5 \mathrm{M} \mathrm{CO}_{3}{ }^{2-}$, and $0.025 \mathrm{M}$ HEDPA. Tests were allowed to equilibrate for $10 \mathrm{~min}$ or $1 \mathrm{~h}$ under gentle agitation at $10 \mathrm{mg} / \mathrm{mL}$ slurry concentrations.

2-10 Sorption of Cs-137 onto vermiculite from 0.0001-0.5 M solutions of $\mathrm{NH}_{4} \mathrm{Cl}, \mathrm{KCl}$, $\mathrm{Na}_{2} \mathrm{CO}_{3}$, and HEDPA or a combined solution of $1 \mathrm{M} \mathrm{K}^{+}, 0.5 \mathrm{M} \mathrm{CO}_{3}^{2-}$, and 0.025 $\mathrm{M}$ HEDPA. Tests were allowed to equilibrate for $1 \mathrm{~h}$ under gentle agitation at $10 \mathrm{mg} / \mathrm{mL}$ slurry concentrations.

2-11 Sorption of Cs-137 onto clays from solution of $0.5 \mathrm{M} \mathrm{KCl}$ after $10 \mathrm{~min}$ and 30 min of gentle agitation.

2-12 Sorption of Cs-137 onto clays from solution of $0.5 \mathrm{M} \mathrm{NH}_{4} \mathrm{Cl}$ after 10 min and 30 min of gentle agitation.

2-13 Kinetics for sorption of Am-241 onto various sorbents from deionized water.

2-14 Sorption of Am-241 onto various sorbents from $\mathrm{KCl}$ solution...................................... 48

2-15 Sorption of Am-241 onto various sorbents from $\mathrm{Na}_{2} \mathrm{CO}_{3}$ solution............................. 48

2-16 Sorption of Am-241 onto various sorbents from HEDPA solution.

3-1 Decontamination of Cs-137 from concrete coupons using 100\% hydrated gel formulations prepared from different wash solutions. Average cesium removal values are shown in increasing removal efficiency for each gel, and triplicate coupon results are represented to the right of each average. Coupons were aged with its contamination for $24 \mathrm{~h}$ at $22^{\circ} \mathrm{C}$ and $40 \% \mathrm{RH}$. 


\section{FIGURES (Cont.)}

3-2 Decontamination of Am-241 and Cs-137 from concrete, brick, and tile coupons using $100 \%$ hydrated gel formulation. Average cesium removal values are shown as data labels in the figure. Individual coupon replicate results are shown to the right of each average in increasing removal efficiency for each substrate. Coupons with its contamination were aged for one week at $40^{\circ} \mathrm{C}$.

4-1 Plume profile used in Liberty RadEx for a hypothetical RDD release..................... 57

4-2 Activity levels for coupons contaminated with Cs-137 and cold cesium carrier. ....... 57

5-1. Test wall containing the nine concrete samples used in the testing at Idaho National Laboratory using the mixed-isotope formulation of the SuperGel.

5-2 Photograph of epoxied coupon side surfaces.

5-3 Photograph showing large pores on surface of concrete coupon.

5-4 Photograph of demonstration test stand and setup: attached coupons, trowel for $1.0 \mathrm{M} \mathrm{KCl}$ gel application, and HEPA-Vac for gel removal.

5-5 Photograph of test sample decontamination using 1.0 M KCl gel formulation for pilot test \#1

5-6 Photograph showing 1.0 M KCl gel removal with HEPA-Vac.

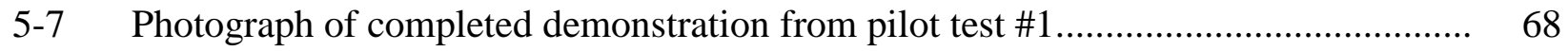

5-8 Photograph showing smooth side of concrete coupon used in pilot test \#2............... 69

5-9 Photograph of coupon decontamination using 1.0 $\mathrm{M} \mathrm{KCl}$ gel formulation for pilot test \#2.

5-10 Tented contamination shelter for Argonne pilot test \#3 with test stand in back of the shelter and double-bagged coupons on cart in front opening of shelter.

5-11 Sprayer wand ready to start gel application on contaminated coupons secured within test stand in tented shelter.

5-12 Sprayer applying gel to contaminated coupons.

5-13 Removal of gel from contaminated coupons using HEPA vacuum during pilot test \#3. 


\section{FIGURES (Cont.)}

5-14 Activity of coupons from Argonne Pilot Test \#1 before and after decontamination. Triplicate counts shown for individual coupons; control coupon activity shown on right.

5-15 Decontamination results for Cs-137 from Argonne Pilot Test \#1 ............................ 75

5-16 Activity of coupons before and after decontamination from Argonne Pilot Test \#2. Triplicate counts shown for individual coupons; control coupon activity shown on right.

5-17 Decontamination results for Cs-137 from Argonne Pilot Test \#2.

5-18 Activity of coupons before and after decontamination from Argonne Pilot Test \#3. Triplicate counts shown for individual coupons; control coupon activity shown on right.

5-19 Decontamination results for Cs-137 from Argonne Pilot Test \#3.

\section{TABLES}

2-1 Test matrix for sequestering agent sorption tests for trace cesium solution................ 6

2-2 Test matrix for sequestering agent sorption tests for trace americium solution. .......... 7

2-3 Summary of the properties of sorbent candidates for cesium in solution.................... 8

2-4 Summary of the properties of sorbent candidates for americium in solution.............. 21

3-1 Polymer hydration capacity for mixed isotope solutions.................................. 51

3-2 Gel formulations of 99:1 cross-linked: linear PAM/30\% PAA prepared at $100 \%$ gel hydration using different wash solutions.

4-1 Cesium removal at low, medium, and high contaminant concentrations from concrete using $100 \%$ hydrated gel formulations. Individual coupon replicate results are shown with average and standard deviations for removal efficiency for each gel formulation.

4-2 Americium removal from concrete using 100\% hydrated gel formulations. Individual coupon replicate results are shown with average and standard deviations for removal efficiency for each gel formulation....

5-1 Summary of Argonne pilot-test conditions for cesium-137 contaminated concrete coupons. 


\section{TABLES (Cont.)}

5-2 Preliminary Results from INL Pilot Test on U.S. Concrete Coupons. ......................... 73

5-3 Summary of cesium removal results on Singapore concrete from Argonne pilot tests. ......................................................................................................... 


\title{
TECHNICAL IMPROVEMENTS TO AN ABSORBING SUPERGEL FOR RADIOLOGICAL DECONTAMINATION IN TROPICAL ENVIRONMENTS
}

\begin{abstract}
Argonne National Laboratory (Argonne) developed a superabsorbing gel-based process (SuperGel) for the decontamination of cesium from concrete and other porous building materials. Here, we report on results that tested the gel decontamination technology on specific concrete and ceramic formulations from a coastal city in Southeast Asia, which may differ significantly from some U.S. sources. Results are given for the evaluation of americium and cesium sequestering agents that are commercially available at a reasonable cost; the evaluation of a new SuperGel formulation that combines the decontamination properties of cesium and americium; the variation of the contamination concentration to determine the effects on the decontamination factors with concrete, tile, and brick samples; and pilot-scale testing $\left(0.02-0.09 \mathrm{~m}^{2}\right.$ or $6-12$ in. square coupons).
\end{abstract}

\section{BACKGROUND}

Argonne National Laboratory (Argonne) developed a superabsorbing gel-based process for the decontamination of cesium from concrete and other porous building materials. [1-3] Argonne was able to develop a technology using commercial spray technology, commercial biocompatible polymers, common chemical reagents, and commercial wet-vacuum technology. The process operations begin with spraying a superabsorbing polymer hydrogel dissolved in a high salt solution onto the contaminated surface. The hydrogel retains its consistency in relatively high temperatures and humidity for periods longer than several hours. The hydrogel is removed using a wet vacuum, and the resultant material can be dehydrated to significantly reduce the waste volume requiring disposal. [1]

Then, we reported on results that tested the gel decontamination technology on specific concrete and ceramic formulations from a coastal city in Southeast Asia, ${ }^{*}$ which may differ significantly from some U.S. sources. [2] We employed bench-scale testing in an attempt to produce an optimized formulation for immediate pilot-scale testing and deployment. The goal was to deploy a hydrogel-decontamination technology that meets the decontamination requirements for the environmental conditions and building materials for such a city.

* The specific city is purposely unidentified. 
Follow-on work sought to fill in the gaps identified in this previous work. [3] Four tasks were completed. [4] In the first task, the depth profile of the contamination was measured and we confirmed the dominating role that porosity plays in the decontamination of brick. Then, we tested a dryer gel product to potentially improve the convective action of the gel and subsequently the removal of pore water from the porous brick structure; we saw no improvement in decontamination factors. Next, we sought to reduce the number of unit operations in the application of the SuperGel technology. We eliminated the first step in the process (i.e., apply wash solution to the surface of the material) and, instead, increased the hydration of the gel to $100 \%$ and found no difference in the percent removal of cesium. In the next task, we examined contaminated concrete samples by $\mathrm{x}$-ray absorption fluorescence spectroscopy to help identify the chemical speciation of residual cesium and americium on samples. Finally, we completed coupon testing of concrete, tile, and brick for the decontamination of americium and made significant progress toward increasing the decontamination of americium from concrete using diphosphonic acid and carbonate ions in the gel. Results from the tile coupons were excellent. Decontamination from brick coupons continued to be problematic due to the high connected porosity.

To reduce the uncertainty with the operational deployment, we have completed four additional tasks and report on these herein. In Task 1 (see Section 2.0), we studied both monosodium titanate (MST) and cellulose acetate as sequestering agents for americium. The original formulations employed crystalline silico-titanate (CST) for cesium (as IONSIVTM IE910, UOP LLC). The cellulose acetate has much poorer affinity for americium than MST. However, MST is no longer commercially available. Similarly, CST has been removed as an off-the-shelf item and has to be custom ordered at a much higher cost. Therefore, we proposed to evaluate other americium and cesium sequestering agents that are commercially available. We performed partitioning coefficient measurements and concrete coupon testing.

In Task 2 (see Section 3.0), we evaluated a SuperGel formulation that combines the decontamination properties of cesium and americium. The SuperGel is compatible with the $\mathrm{KCl}$ or $\mathrm{NH}_{4} \mathrm{Cl}$, and $\mathrm{Na}_{2} \mathrm{CO}_{3}$ and 1-hydroxyethylidene diphosphonic acid (HEDPA) reagents, thereby expanding its applicability to the decontamination of trivalent actinides (and lanthanides, by inference, since they are similarly trivalent in water and display nearly identical behavior as hard Lewis acids), and likely to divalent cations as well, since the HEDPA has a high affinity to those oxidation states in solution.

In Task 3 (see Section 4.0), we varied the concentration of contamination to determine any effects on the decontamination factors. Concrete, tile, and brick samples were tested.

In Task 4 (see Section 5.0), we expanded the decontamination testing beyond our benchtop experiments that used small quantities of substrate material. This pilot-scale test involved larger test samples $\left(0.02-0.09 \mathrm{~m}^{2}\right.$ or $6-12$ in. square coupons) contaminated to levels consistent with previous testing. The SuperGel would be applied using a variety of methods that can be employed in the field, including industrial sprayers and vacuums rated for radioactive materials. 


\section{REPLACEMENT SEQUESTERING AGENTS TO MST AND CST (TASK 1)}

\subsection{INTRODUCTION}

We had previously studied both MST, a synthetic zeolite designed for the removal of radionuclides from liquid high-level nuclear waste, and cellulose acetate as sequestering agents for americium. [3] We found that cellulose acetate has much poorer affinity for americium than MST. However, MST is no longer commercially available. Moreover, CST is no longer an offthe-shelf item and has to be custom-ordered at a much higher cost. Therefore, we evaluated other americium and cesium sequestering agents that are commercially available. This study determined the sorption behavior of soluble cesium and americium from solution onto synthetic and natural ion-exchange materials.

\subsection{METHODS}

\subsubsection{Materials}

An in-house stock solution of ${ }^{137} \mathrm{Cs}$ was used for the sorption experiments. Americium-241 was purchased from Eckert \& Ziegler Isotope Products $(1 \mu \mathrm{Ci} / \mathrm{mL}$, June 1, 2008) and diluted into deionized water before spiking (usually $10 \mu \mathrm{L}$ ) into each stock solution tested. To test for cesium sorption, we employed:

- $\quad$ CST (IONSIVTM-IE910, Universal Oil Products)

- KMS-2 [K $\mathrm{K}_{2 \mathrm{x}} \mathrm{Mn}_{x} \mathrm{Sn}_{3-\mathrm{x}} \mathrm{S}_{6}(x=0.5-0.95)$, Northwestern University], a layered sulfide mineral that is not available in the market but uses a synthesis method that the inventors believe may be cost-effective in the long term

- vermiculite concentrate, a natural smectite with general formula $\left.(\mathrm{Mg}, \mathrm{Fe}, \mathrm{Al})_{3}(\mathrm{Al}, \mathrm{Si})_{4} \mathrm{O}_{10}(\mathrm{OH})_{2} \cdot 4 \mathrm{H}_{2} \mathrm{O}\right)(\mathrm{W} . \mathrm{R}$. Grace \& Co.)

- $\quad$ illite (Xuzhou Zhonglian Chemical Technology Co., Ltd.)

- chabazite $\left[\left(\mathrm{Ca}, \mathrm{Na}_{2}, \mathrm{~K}_{2}, \mathrm{Mg}\right) \mathrm{Al}_{2} \mathrm{Si}_{4} \mathrm{O}_{12} \cdot 6 \mathrm{H}_{2} \mathrm{O}, \mathrm{ZC} 500 \mathrm{H},-50\right.$ mesh, St. Cloud Mining Co.; Winston, New Mexico]

As negative controls, we used diatomaceous earth $\left(\mathrm{SiO}_{2}\right.$, crystalline form, Celite Corporation) obtained from Separmatic Filter (Menomonee Falls, Wisconsin). 
We completed sorption experiments for ${ }^{241} \mathrm{Am}$ onto various potential replacement sequestering agents for MST. These included:

- clinoptilolite, a natural zeolite with general formula (Na,K,Ca) ${ }_{3} \mathrm{Al}_{3}(\mathrm{Al}, \mathrm{Si})_{2} \mathrm{Si}_{13} \mathrm{O}_{36} \cdot 12 \mathrm{H}_{2} \mathrm{O}$ (St. Cloud Mining Co., New Mexico)

- montmorillonite, a natural smectite with general formula $(\mathrm{Na}, \mathrm{Ca})_{0.33}(\mathrm{Al}, \mathrm{Mg})_{2}\left(\mathrm{Si}_{4} \mathrm{O}_{10}\right)(\mathrm{OH})_{2} \cdot n \mathrm{H}_{2} \mathrm{O}$ (Fischer Scientific USA as K-10)

- $\quad$ vermiculite (same as above)

- birnessite, an manganese oxide mineral with general formula $\left(\mathrm{Na}_{0.3} \mathrm{Ca}_{0.1} \mathrm{~K}_{0.1}\right)\left(\mathrm{Mn}^{4+}, \mathrm{Mn}^{3+}\right)_{2} \mathrm{O}_{4} \cdot 1.5 \quad \mathrm{H}_{2} \mathrm{O} \quad$ (synthesized by Ohio State University, Prof. N. Basta)

The $\mathrm{pH}$ of the solution was adjusted in some experiments by addition of sodium hydroxide (diluted from pellets, 99.998\%, Aldrich) or nitric acid (0.1 M). Solutions of ammonium chloride $\left(\mathrm{NH}_{4} \mathrm{Cl}, 99.5+\%\right.$, A.C.S. reagent, Sigma-Aldrich), potassium chloride (KCl, Analytical AR Reagent, Mallinckrodt), sodium carbonate $\left(\mathrm{Na}_{2} \mathrm{CO}_{3}\right.$, anhydrous, granular AR ACS Primary Standard, Mallinckrodt), and etidronic acid (hydroxyethane diphosphonic acid, Aldrich) were prepared with deionized $\mathrm{H}_{2} \mathrm{O}\left(\sim 18 \mathrm{M} \Omega \bullet \mathrm{cm}\right.$ resistivity at $\left.25^{\circ} \mathrm{C}\right)$. Standard buffer solutions ( $\mathrm{pH}=4.01,7.01$, and 10.01) were used to calibrate a $\mathrm{pH}$ meter (Hanna Instruments).

Material was weighed into $1.5 \mathrm{~mL}$ microcentrifuge tubes (Ambion). All experiments were carried out in triplicate, and duplicate aliquots were taken from each sample. Gamma counting was recorded with a Minaxi $\gamma$ Auto-Gamma 5000 Series Gamma Counter (United Technologies Packard, Model A5550) using $12 \times 75$ polypropylene tubes.

Following Kandil et al., ${ }^{1}$ illite and vermiculite were washed with deionized water (DIW) and centrifuged four times to remove any dust or water-soluble impurities. The supernatant was removed with a disposable transfer pipette after each centrifugation and replaced with new DIW. Each washed sample was placed in a glass petri dish and dried in an oven at $398 \mathrm{~K}$ for 22 hours. Samples were then placed in a dessicator.

\subsubsection{Determination of pH Dependence}

We performed $\mathrm{pH}$-dependent measurements on the partitioning of cesium onto potential replacement materials. The procedure was as follows: weigh $10 \mathrm{mg}$ of CST, illite, KMS-2, or vermiculite and place it into $1.5 \mathrm{~mL}$ microcentrifuge tubes. Add $1.0 \mathrm{~mL}$ of the following solutions: $0.01 \mathrm{M} \mathrm{KCl}$ to $\mathrm{CST}$, and $1.0 \mathrm{M} \mathrm{HNO}_{3}$ solution at $\mathrm{pH} 4$ to illite, $\mathrm{KMS}-2$, and vermiculite. Add $10 \mu \mathrm{L} \mathrm{HNO}_{3}$ to samples and agitate in a rotating mixer for 60 min until the pH is lowered to $\mathrm{pH} 3$ or 4 . 


\subsubsection{Kinetic Experiments}

We completed kinetic experiments to determine the sorption of cesium and americium onto the various sequestering agents as a function of time in deionized water. The distribution coefficient, $K_{d}$, is calculated according to the equation:

$$
K_{d}=\left(C_{0}-C_{f}\right) / C_{f} * V / m
$$

where $C_{0}$ and $C_{f}$ are the initial and equilibrium radionuclide activity, $V$ is the volume of solution in milliliters, and $m$ is the mass of the sorbent in grams. We prepared solutions for ${ }^{137} \mathrm{Cs}$ and ${ }^{241} \mathrm{Am}$ sorption tests and calibrated pipets to the specified volume. We then placed $10 \mathrm{mg}$ of sorbent material in each microcentrifuge tube and added $1.0 \mathrm{~mL}$ of radioactive spiked solution. Each microcentrifuge tube was capped and gently mixed on a rotator mixer for one hour. The timer was started once the radioactive spike was added to the solution. Samples were mixed for the indicated contamination time up to $24 \mathrm{~h}$. Triplicate test tubes were centrifuged for five minutes at $6000 \mathrm{rpm}$. We took duplicate $400 \mu \mathrm{L}$ aliquots from each microcentrifuge tube for gamma analysis [Minaxi Gamma Counter, model A5550, 3 in. well-type $\mathrm{Na}(\mathrm{I})$ crystal] of the region from $500-900 \mathrm{keV}$ ( $\gamma$-energy for ${ }^{137} \mathrm{Cs}$ is $662 \mathrm{keV}$ ). The control consisted of a blank microcentrifuge tube mixed and sampled as described above for each time period.

\subsubsection{Sorption of Cesium from Solution}

We tested the sorption of cesium from a mixed formulation solution of $1 \mathrm{M} \mathrm{K}^{+}$, $0.5 \mathrm{M} \mathrm{CO}_{3}{ }^{2-}$, and $0.025 \mathrm{M}$ HEDPA onto natural mineral sorbents: chabazite, montmorillonite, and vermiculite. Tests were run in triplicate at $10 \mathrm{mg} / \mathrm{mL}$ and sampled for gamma analysis with duplicate aliquots. A triplicate set of blanks containing no sorbent was run under the same test conditions. Samples were agitated gently in a rotisserie shaker (Barnstead/Thermolyne Labquake Shaker Rotisserie) for one hour. Sample aliquots were either 100 or $400 \mu \mathrm{L}$ and were counted for the $662 \mathrm{keV}$ photopeak of Cs-137 using a NaI spectrometer (Packard Minaxi AutoGamma, Model A5550, $4 \pi$ crystal) for ten minutes.

\subsubsection{Solid-to-Liquid Ratio}

We completed tests of the equilibrium partitioning coefficient $\left(\mathrm{K}_{\mathrm{d}}\right)$ as a function of the sorbent clay slurry concentration and type of sorbent. Sorption was in the presence of $0.5 \mathrm{M} \mathrm{KCl}$ or $\mathrm{NH}_{4} \mathrm{Cl}$ using 10-125 mg sorbent/mL salt solution after mixing for 10 and $30 \mathrm{~min}$ (triplicate tests and duplicate aliquots for analysis).

We summarize the test matrix for cesium and americium sorption in Tables 2-1 and 2-2, respectively. 
TABLE 2-1 Test matrix for sequestering agent sorption tests for trace cesium solution.

\begin{tabular}{|c|c|c|c|c|c|c|}
\hline $\begin{array}{c}\text { Test } \\
\text { Series } \\
\end{array}$ & $\begin{array}{c}\# 1 \text { - Kinetic } \\
\text { tests }\end{array}$ & $\begin{array}{c}\# 2 \text { - Effect of } \\
\mathrm{pH}\end{array}$ & $\begin{array}{c}\# 3 \text { - Effect of } \\
\mathrm{K}^{+} \\
\end{array}$ & $\begin{array}{c}\# 4-\text { Effect of } \\
\mathrm{NH}_{4}^{+} \\
\end{array}$ & $\begin{array}{c}\# 5-\text { Effect of } \\
\mathrm{CO}_{3}{ }^{2-} \\
\end{array}$ & $\begin{array}{c}\# 6 \text { - Effect of } \\
\text { HEDPA }\end{array}$ \\
\hline Sorbent & $\begin{array}{l}\text { control (none), } \\
\mathrm{SiO}_{2}, \mathrm{CST}, \\
\text { vermiculite, } \\
\text { illite }\end{array}$ & $\begin{array}{l}\text { control } \\
\text { (none), } \mathrm{SiO}_{2} \text {, } \\
\text { KMS-2, } \\
\text { CST, } \\
\text { vermiculite, } \\
\text { illite }\end{array}$ & $\begin{array}{l}\text { control (none), } \\
\mathrm{SiO}_{2}, \mathrm{KMS}-2 \text {, } \\
\mathrm{CST}, \\
\text { vermiculite, } \\
\text { illite, chabazite }\end{array}$ & $\begin{array}{l}\text { control (none), } \\
\mathrm{SiO}_{2}, \mathrm{KMS}-2 \text {, } \\
\mathrm{CST} \text {, } \\
\text { vermiculite, } \\
\text { illite, chabazite }\end{array}$ & $\begin{array}{l}\text { control (none), } \\
\mathrm{SiO}_{2}, \mathrm{CST}, \\
\text { vermiculite, } \\
\text { illite, chabazite }\end{array}$ & $\begin{array}{l}\text { control (none), } \\
\mathrm{SiO}_{2}, \mathrm{CST} \text {, } \\
\text { vermiculite, } \\
\text { illite, chabazite }\end{array}$ \\
\hline Solution & $\begin{array}{l}\text { deionized water } \\
\text { at } \mathrm{pH}=8 \\
\text { (adjust with } \\
\text { ultrapure } \\
\mathrm{NaOH} \text { or } \\
\mathrm{HNO}_{3} \text { ) }\end{array}$ & $\begin{array}{l}\mathrm{pH}=4,6,8, \\
10 \text { (deionized } \\
\text { water, pH } \\
\text { adjusted with } \\
\text { ultrapure } \\
\mathrm{NaOH} \text { or } \\
\mathrm{HNO}_{3} \text { ) }\end{array}$ & $\begin{array}{l}{[\mathrm{KCl}]=0,1 \mathrm{e}-4,} \\
0.001,0.01, \\
0.1, \text { and } 0.5 \mathrm{M}\end{array}$ & $\begin{array}{l}{\left[\mathrm{NH}_{4} \mathrm{Cl}\right]=0,} \\
1 \mathrm{e}-4,0.001 \text {, } \\
0.01,0.1 \text {, and } \\
0.5 \mathrm{M}\end{array}$ & $\begin{array}{l}{\left[\mathrm{Na}_{2} \mathrm{CO}_{3}\right]=0,} \\
1 \mathrm{e}-4,0.001, \\
0.01,0.1, \text { and } \\
0.5 \mathrm{M}\end{array}$ & $\begin{array}{l}{[\text { HEDPA }]=0 \text {, }} \\
1 \mathrm{e}-4,0.001 \text {, } \\
0.01,0.1 \text {, and } \\
0.5 \mathrm{M}\end{array}$ \\
\hline Time & $\begin{array}{l}5,15,30, \\
60 \text { min, and } \\
\text { overnight } \\
\text { (record actual } \\
\text { time); Note: for } \\
\text { tests at } t=5 \\
\text { and } 15 \text { min } \\
\text { freeze in ice } \\
\text { bath until ready } \\
\text { to sample }\end{array}$ & $1 \mathrm{~h}$ & $1 \mathrm{~h}$ & $1 \mathrm{~h}$ & $1 \mathrm{~h}$ & $1 \mathrm{~h}$ \\
\hline $\begin{array}{l}\text { Clay } \\
\text { mass to } \\
\text { solution } \\
\text { volume } \\
\text { ratio }\end{array}$ & $10 \mathrm{mg} / \mathrm{mL}$ & $10 \mathrm{mg} / \mathrm{mL}$ & $\begin{array}{l}10 \mathrm{mg} / \mathrm{mL} ; 10, \\
40,75,100, \\
125 \mathrm{mg} / \mathrm{mL} \text { for } \\
0.5 \mathrm{M} \mathrm{KCl} \text { only }\end{array}$ & $\begin{array}{l}10 \mathrm{mg} / \mathrm{mL} ; 10 \\
40,75,100 \\
125 \mathrm{mg} / \mathrm{mL} \\
\text { for } 0.5 \mathrm{M} \\
\mathrm{NH}_{4} \mathrm{Cl} \text { only }\end{array}$ & $10 \mathrm{mg} / \mathrm{mL}$ & $10 \mathrm{mg} / \mathrm{mL}$ \\
\hline
\end{tabular}


TABLE 2-2 Test matrix for sequestering agent sorption tests for trace americium solution (clay mass-to-solution-volume ratio $=10 \mathrm{~mL} / \mathrm{g}$ ).

\begin{tabular}{|c|c|c|c|c|c|}
\hline $\begin{array}{c}\text { Test } \\
\text { Series } \\
\end{array}$ & $\# 1$ - Kinetic tests & $\# 2$ - Effect of $\mathrm{K}^{+}$ & $\begin{array}{c}\# 3 \text { - Effect of } \\
\mathrm{NH}_{4}{ }^{+}\end{array}$ & $\begin{array}{c}\# 4-\text { Effect of } \\
\mathrm{CO}_{3}{ }^{2-} \\
\end{array}$ & $\begin{array}{c}\# 5 \text { - Effect of } \\
\text { HEDPA }\end{array}$ \\
\hline Sorbent & $\begin{array}{l}\text { control (no } \\
\text { sorbent), } \\
\text { birnessite, } \\
\text { montmorillonite, } \\
\text { clinoptilolite, } \\
\text { vermiculite }\end{array}$ & $\begin{array}{l}\text { control (no } \\
\text { sorbent), } \\
\text { birnessite, } \\
\text { montmorillonite, } \\
\text { clinoptilolite, } \\
\text { vermiculite }\end{array}$ & $\begin{array}{l}\text { control (no } \\
\text { sorbent), } \\
\text { birnessite, } \\
\text { montmorillonite, } \\
\text { clinoptilolite, } \\
\text { vermiculite }\end{array}$ & $\begin{array}{l}\text { control (no } \\
\text { sorbent), } \\
\text { birnessite, } \\
\text { montmorillonite, } \\
\text { clinoptilolite, } \\
\text { vermiculite }\end{array}$ & $\begin{array}{l}\text { control (no } \\
\text { sorbent), } \\
\text { birnessite, } \\
\text { montmorillonite, } \\
\text { clinoptilolite, } \\
\text { vermiculite }\end{array}$ \\
\hline Solution & $\begin{array}{l}\text { deionized water at } \\
\mathrm{pH}=8 \text { (adjust } \\
\text { with ultrapure } \\
\mathrm{NaOH} \text { or } \mathrm{HNO}_{3} \text { ) }\end{array}$ & $\begin{array}{l}{[\mathrm{KCl}]=0,1 \mathrm{e}-4,} \\
0.001,0.01,0.1, \\
\text { and } 0.5 \mathrm{M}\end{array}$ & $\begin{array}{l}{\left[\mathrm{NH}_{4} \mathrm{Cl}\right]=0,1 \mathrm{e}-} \\
4,0.001,0.01 \text {, } \\
0.1 \text {, and } 0.5 \mathrm{M}\end{array}$ & $\begin{array}{l}{\left[\mathrm{Na}_{2} \mathrm{CO}_{3}\right]=0,} \\
1 \mathrm{e}-4,0.001,0.01, \\
0.1 \text {, and } 0.5 \mathrm{M}\end{array}$ & $\begin{array}{l}{[\mathrm{HEDPA}]=0,} \\
1 \mathrm{e}-4,0.001,0.01 \text {, } \\
0.1 \text {, and } 0.5 \mathrm{M}\end{array}$ \\
\hline Time & $\begin{array}{l}5,15,30,60 \mathrm{~min} \text {, } \\
\text { and overnight } \\
\text { (record actual } \\
\text { time); for tests at } \\
\mathrm{t}=5 \text { and } 15 \mathrm{~min} \text {, } \\
\text { freeze in ice bath } \\
\text { until ready to } \\
\text { sample }\end{array}$ & $1 \mathrm{~h}$ & $1 \mathrm{~h}$ & $1 \mathrm{~h}$ & $1 \mathrm{~h}$ \\
\hline
\end{tabular}

\subsection{RESULTS}

We have been surveying replacement sequestering agents for MST and CST that offer high affinity for Am and Cs, are readily available, are non-toxic, and are inexpensive. Results are given below.

\subsubsection{Properties of Candidates for Sequestering Agent Replacements}

We studied a number of natural and synthetic ion-exchange materials and sorbents by perusing the literature first. We searched for studies that reported on the sorption of cesium or americium onto solid sorbents with an emphasis on studies using high ionic strength solutions since the SuperGel is reconstituted in molarity levels of salt. Sorbent properties were reviewed as a function of important sorption properties: $\mathrm{pH}$, equilibration time, material surface area or size, cation exchange capacity, and solution composition. We did not include every study or sorbent we reviewed, because the list would have been prohibitively long. Instead, we selected representative materials as a means of representing the data from many authors. For instance, we did not report on every study on montmorillonite clay; but instead, we reported on studies that represent the rest of the literature as typical values. In Table 2-3, we summarize the literature on sorbents for cesium. In Table 2-4, we summarize it for sorbents for americium. 


\section{TABLE 2-3 Summary of the properties of sorbent candidates for cesium in solution.}

\begin{tabular}{|c|c|c|c|c|c|c|c|}
\hline Sorbent Material & $\begin{array}{c}\text { Concentration } \\
\text { of Element }\end{array}$ & $\begin{array}{c}\mathrm{K}_{\mathrm{d}} \text { in } \mathrm{mL} / \mathrm{g} \\
\text { (or other units or } \\
\% \text { sorbed) }\end{array}$ & CEC (meq/100g) & $\begin{array}{l}\text { BET Area }\left(\mathrm{m}^{2} / \mathrm{g}\right) \\
\text { or Size (microns) }\end{array}$ & Solution Conditions & Notes & Reference \\
\hline $\begin{array}{l}\text { Magnetite, Goethite, } \\
\text { Hematite, Limonite, } \\
\text { Ilmenite, Diaspore } \\
(\mathrm{Al}(\mathrm{OH}) \mathrm{O}), \text { Enstatite, } \\
\text { Quartz, Dolomite, } \\
\text { bauxite called Mixed } \\
\text { Minerals (MM) }\end{array}$ & $\begin{array}{l}\text { Tracer, } \\
\text { concentration } \\
\text { or activity not } \\
\text { stated }\end{array}$ & $\begin{array}{l}\text { Almost } 100 \% \\
\text { adsorption Cs and } \\
\text { Sr at } 200 \mathrm{mg} \text { solid } \\
\text { loading (no liq. } \\
\text { Volume given) }\end{array}$ & $\begin{array}{l}35 \mathrm{~g} / \mathrm{kg} \mathrm{Cs} \\
(0.25 \mathrm{mmol} / \mathrm{g}) \\
\text { loading capacity } \\
\text { and } 25 \mathrm{~g} / \mathrm{kg} \text { for } \\
\mathrm{Sr}(0.27 \mathrm{mmol} / \mathrm{g})\end{array}$ & $\begin{array}{l}80-100 \text { mesh } \\
(0.044-0.74 \mathrm{~mm}) \\
\text { without } \\
\text { pretreatment }\end{array}$ & $\begin{array}{l}\mathrm{pH} 1-14 \text { and as function of } \\
\text { nitric, perchloric, and sulfuric } \\
\text { acids. Used } 10 \mathrm{mg}-5 \mathrm{~g} \text { solid in } \\
\text { liquid (volume not stated) }\end{array}$ & $\begin{array}{l}\text { Adsorption of } 137 \mathrm{Cs} \text { and } 90 \mathrm{Sr} \\
\text { radionuclides on MM from aq. solns. is } \\
\text { reported by describing the effect of } \\
\text { equilibration time. } \mathrm{H} \text { ions, and } \\
\text { adsorbent and adsorbate concn. } \\
\text { Optimum physicochem. conditions } \\
\text { were identified. }\end{array}$ & $\begin{array}{l}\text { Ahmad } \\
1989\end{array}$ \\
\hline soil & $?$ & $99 \%$ removal & & & & $\begin{array}{l}\text { mix Cs } 137 \text { contaminated soil with } \\
\text { chloride and heat }\left(800-1000^{\circ} \mathrm{C}\right) \text { for } 2 \mathrm{~h}\end{array}$ & Spalding \\
\hline $\begin{array}{l}\text { askanite, gumbrin, } \\
\text { nontronite, bentonite, } \\
\text { kaolin, phlogopite, } \\
\text { biotite, muscorite, } \\
\text { talcum, vermiculite, } \\
\text { glauconite, } \\
\text { hydrophlogopite, } \\
\text { hydromuscovite, } \\
\text { hydrogoethite, or } \\
\text { virianite }\end{array}$ & $\begin{array}{l}\text { details in } \\
\text { paper? }\end{array}$ & 66.5 to $99.9 \%$ & & & details in paper & $\begin{array}{l}\text { Increased sorption of } 137 \mathrm{Cs} \text { from } 37.1 \\
\text { with only sand to following with } \\
\text { addition of } \sim 1 \text { wt } \% \text { minerals: } 99.9 \text { - } \\
\text { askanite, } 99.9 \text {-gumbrin, 99.9- } \\
\text { nontronite, 97.0-bentonite, 95.6-kaolin, } \\
\text { 99.7-phlogopite, 97.3-biotite, } 94.5 \text { - } \\
\text { muscorite, } 87.2 \text {-talcum, 99.8- } \\
\text { vermiculite, } 98.3 \text {-glauconite, } 99.6- \\
\text { hydrophlogopite, } 88.9 \text {-hydromuscovite, } \\
\text { 66.5-hydrogoethite, and 98.9-virianite; } \\
\text { NH4NO3, NaNO3, and Cs(NO3)2 also } \\
\text { desorbed } 137 \mathrm{Cs} \text { from minerals and the } \\
\text { desorption increased as the radius of } \\
\text { the displacing ion approached the ionic } \\
\text { radius of 137Cs. }\end{array}$ & $\begin{array}{l}\text { Bakunov } \\
1989\end{array}$ \\
\hline $\begin{array}{l}\text { layered metal sulfides, } \\
\text { KMS-1 (synthesized } \\
\text { material) }\end{array}$ & $30-5 e 3 p p b$ & 150 to 21,000 & $\begin{array}{l}226 \mathrm{mg} \mathrm{Cs} / \mathrm{g} \text { or } 1.7 \\
\mathrm{mmol} \mathrm{Cs} / \mathrm{g}\end{array}$ & & $\begin{array}{l}\mathrm{t}=12 \mathrm{~h}, \mathrm{pH}=1-12, \mathrm{~V} / \mathrm{m}=50- \\
1000 \mathrm{~mL} / \mathrm{g}, \mathrm{RT}, \text { molar ratios } \\
\text { of } \mathrm{Na} / \mathrm{Cs}: 0-2 \mathrm{e} 4, \mathrm{Ca} / \mathrm{Cs}: 0-3 \mathrm{e} 3 \\
\text { and } \mathrm{Mg} / \mathrm{Cs}: 0-1 \mathrm{e} 3\end{array}$ & 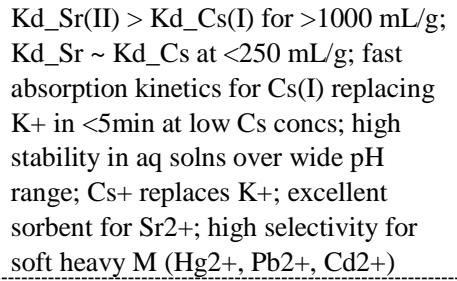 & Manos 2009 \\
\hline
\end{tabular}


TABLE 2.3 (Cont.)

\begin{tabular}{|c|c|c|c|c|c|c|c|}
\hline$---\cdot$ & $\begin{array}{c}\text { Concentration } \\
\text { of Element }\end{array}$ & $\begin{array}{c}\mathrm{K}_{\mathrm{d}} \text { in } \mathrm{mL} / \mathrm{g} \\
\text { (or other units or } \\
\% \text { sorbed) }\end{array}$ & CEC (meq/100g) & $\begin{array}{l}\text { BET Area }\left(\mathrm{m}^{2} / \mathrm{g}\right) \\
\text { or Size (microns) }\end{array}$ & Solution Conditions & Notes & Reference \\
\hline $\begin{array}{l}\text { zeolites, sirconium- } \\
\text { titanium silicates }\end{array}$ & & & $\begin{array}{l}1.86 \text { to } 4.1 \mathrm{mmol} \\
\mathrm{Cs} / \mathrm{g}\end{array}$ & & & $\begin{array}{l}\text { most efficient Cs sorbents; refs therein } \\
\text { [Moller et al. 2001, J. Mater. Chem; } \\
\text { Chang et al. } 1998 \text { Ind. Eng. Chem. } \\
\text { Res.; Bortun et al., 2000, Chem. } \\
\text { Mater.; Clearfield et al. } 1998 \text { J. Mol. } \\
\text { Struct.] }\end{array}$ & Manos 2009 \\
\hline perlite & & & & & & $\begin{array}{l}\text { The present invention relates to } \\
\text { treatment of chem. and radioactive } \\
\text { waste contg. cesium and/or rubidium } \\
\text { by introducing it to the ceramic } \\
\text { matrixes sintered contg. expanded } \\
\text { pearlite. The process includes primarily } \\
\text { the stages consisting in prepg. a soln. } \\
\text { of salt of cesium and/or rubidium; } \\
\text { adding the expanded pearlite in desired } \\
\text { quantity in order to absorb the totality } \\
\text { or near total of the prepd. soln.; } \\
\text { elimination solvent from the mass } \\
\text { obtained; precalcination the compd. of } \\
\text { pearlite obtained to form the powder of } \\
\text { intermediate compd. prepd. at the } \\
\text { preceding stages and carrying out the } \\
\text { pearlite contg. cesium and/or rubidium. }\end{array}$ & $\begin{array}{l}\text { Rehspringer } \\
2004\end{array}$ \\
\hline $\begin{array}{l}\text { bentonite clays } \\
\text { (Na-form by treatmt w } \\
\text { Na2CO3, from } \\
\text { Khakassiya deposit, } \\
\text { Russia) }\end{array}$ & $5.2 \mathrm{e}-5 \mathrm{M}$ & 15 to $90 \%$ & 27 & $\begin{array}{l}29 \mathrm{~m} 2 / \mathrm{g} ; 3.16 \mathrm{um} \\
+50 \mathrm{~nm} \text { colloids }\end{array}$ & $\begin{array}{l}\mathrm{IS}=0.001 \text { to } 0.1 \mathrm{M},[\mathrm{Cs}]=5.2 \mathrm{e}- \\
5 \mathrm{M}, \mathrm{pH}=1.5 \text { to } 10\end{array}$ & $\begin{array}{l}\text { Table } 1 \text { gives mineral composition of } \\
\text { bentonite used ( } 70-80 \% \\
\text { montmorillonite); at } \mathrm{pH}>6 \text { and } \mathrm{I}= \\
0.001 \mathrm{M},>90 \% \text { sorbed; dec sorption at } \\
\text { higher I and acidic conditions; } \\
\text { bentonite equilibrated soln: } 300 \mathrm{ppm} \\
\mathrm{Na}+, 50 \mathrm{ppm} \mathrm{CO} / \mathrm{HCO} 3,45 \mathrm{ppm} \mathrm{SO} 4 \\
\text { (refer to Table 2), NaClO4 for I } \\
\text { adjustmt, pH adjusted w } \mathrm{HCl} \text { or } \\
\mathrm{NaOH} \text {; sorption of } \mathrm{Cs} \text { predominantly } \\
\text { ion-exchg - strong dep on ionic } \\
\text { strength; also contains data for Pu }\end{array}$ & $\begin{array}{l}\text { Sabodina } \\
2006\end{array}$ \\
\hline
\end{tabular}


TABLE 2.3 (Cont.)

\begin{tabular}{|c|c|c|c|c|c|c|c|}
\hline Sorbent Material & $\begin{array}{c}\text { Concentration } \\
\text { of Element }\end{array}$ & $\begin{array}{c}\mathrm{K}_{\mathrm{d}} \text { in } \mathrm{mL} / \mathrm{g} \\
\text { (or other units or } \\
\% \text { sorbed) }\end{array}$ & $\mathrm{CEC}(\mathrm{meq} / 100 \mathrm{~g})$ & $\begin{array}{l}\text { BET Area }\left(\mathrm{m}^{2} / \mathrm{g}\right) \\
\text { or Size (microns) }\end{array}$ & Solution Conditions & Notes & Reference \\
\hline $\begin{array}{l}\text { hydrous manganese } \\
\text { oxide (synthesized matl) }\end{array}$ & $\begin{array}{l}1 \mathrm{e}-2 \text { to } 1 \mathrm{e}-8 \mathrm{M} \\
+ \text { trace } \mathrm{Cs} 134\end{array}$ & $1 \mathrm{e} 3 \mathrm{~mL} / \mathrm{g}$ & & 120-170 mesh & $\begin{array}{l}0.1 \mathrm{~g} \text { sorbent } / 10 \mathrm{~mL} \text { soln, } \mathrm{t}=0- \\
2 \mathrm{~h}, \mathrm{pH}=3.85\end{array}$ & & $\begin{array}{l}\text { Mishra } \\
2007\end{array}$ \\
\hline $\begin{array}{l}\text { hydrous stannic oxide } \\
\text { (synthesized matl) }\end{array}$ & $\begin{array}{l}1 \mathrm{e}-2 \text { to } 1 \mathrm{e}-8 \mathrm{M} \\
+ \text { trace } \mathrm{Cs} 134\end{array}$ & $1 \mathrm{e} 3 \mathrm{~mL} / \mathrm{g}$ & & $120-170$ mesh & $\begin{array}{l}0.1 \mathrm{~g} \text { sorbent } / 10 \mathrm{~mL} \text { soln, } \mathrm{t}=0- \\
2 \mathrm{~h}, \mathrm{pH}=3.85\end{array}$ & & $\begin{array}{l}\text { Mishra } \\
2007\end{array}$ \\
\hline $\begin{array}{l}\text { bentonite } \\
(\mathrm{Na} 0.3(\mathrm{AlMg}) 2 \mathrm{Si} 4 \mathrm{O} 10 \\
(\mathrm{OH}) 2-4 \mathrm{H} 2 \mathrm{O}) \text { and pink } \\
\text { granite }\end{array}$ & $\begin{array}{l}\text { tracer to } 0.01 \mathrm{M} \\
\text { Cs/Sr. tracer to } \\
10^{\wedge}-5 \mathrm{M} \mathrm{Am} .\end{array}$ & $\begin{array}{l}\mathrm{Kd}(\mathrm{Cs}- \\
\text { bentonite })=\sim 600 \mathrm{~mL} \\
/ \mathrm{g} ; \mathrm{Kd}(\mathrm{Cs}- \\
\text { granite })=24 \mathrm{~mL} / \mathrm{g} \text { at } \\
\mathrm{pH} 7-8, \text { increases at } \\
\mathrm{pH}>9 . \mathrm{Kd}(\mathrm{Am}- \\
\text { bentonite })=\sim 4000 \mathrm{~m} \\
\mathrm{~L} / \mathrm{g} \text { at } \mathrm{pH} 7-8 . \\
\mathrm{Kd}(\mathrm{Cs}- \\
\text { bentonite })=\sim 110 \mathrm{~mL} \\
/ \mathrm{g} \text { at } 0.5 \mathrm{M} \mathrm{Na}+ \\
/ \mathrm{Ca} 2+\text {. }\end{array}$ & $\begin{array}{l}0.72 \text { and } 0.33 \\
\mathrm{mmol} / \mathrm{g} \text { for Cs and } \\
\text { Sr sorption capacity } \\
\text { by Langmuir }\end{array}$ & $\begin{array}{l}\text { Bentonite fine } \\
\text { powder; granite } \\
70-100 \text { mesh }\end{array}$ & $\begin{array}{l}400: 1 \text { to } 20: 1 \text { volume to mass } \\
\text { ratio for borehole water with } \\
\text { pH 2-10. } 0.01 \mathrm{M} \text { to } 0.5 \mathrm{M} \text { salt }\end{array}$ & $\begin{array}{l}\text { Studied ability of backfill and host } \\
\text { granite host rock to sorb radionuclides. } \\
30 \text { min to reach equilirbium for } \\
\text { bentonite and Cs. } 60 \text { min for granite. }\end{array}$ & Murali 2002 \\
\hline cements & & $0.1-100$ & & & & reported $\mathrm{Kd}$ as $1 \mathrm{e}-4$ to $0.1 \mathrm{~m} 3 / \mathrm{kg}$ & $\begin{array}{l}\text { McKinley } \\
1993\end{array}$ \\
\hline soil/surface sediments & & 10,000 & & & & reported $\mathrm{Kd}$ as $10 \mathrm{~m} 3 / \mathrm{kg}$ & $\begin{array}{l}\text { McKinley } \\
1993\end{array}$ \\
\hline $\begin{array}{l}\text { montmorillonite, No. } 11 \\
\text { (sodium saturated form, } \\
\text { Santa Rita, NM } \\
\text { deposits) }\end{array}$ & $\begin{array}{l}10-10-0.1 \mathrm{~N}+ \\
\text { trace } \mathrm{Cs} 137\end{array}$ & $\begin{array}{l}50,000[\text { low } \\
\mathrm{Na}+\mathrm{Cs}] 10[\mathrm{hi} \\
\mathrm{Cs}+\mathrm{Na}]\end{array}$ & 116 & $<100$ mesh & $\begin{array}{l}\mathrm{V} / \mathrm{m}=100 \mathrm{~mL} / \mathrm{g}, 100 \mathrm{mg} \text { clay } / \\
10 \mathrm{~mL},[\mathrm{Na}]=0.2-0.002 \mathrm{~N} \\
\mathrm{t}=12 \mathrm{~h}, \mathrm{pH}=?\end{array}$ & $\begin{array}{l}\text { exchg capacity due to isomorphous } \\
\text { substitutions in crystal lattice (per Ref } \\
\text { therein, Lewis-1955, p 54-55); } \\
\text { Montmorillonite characterized from } \\
\text { Santa Rita, NM as } \\
\text { (Na,Ca)0.3(Al,Mg)2Si4O10(OH)2.nH } \\
2 \mathrm{O} \text { per http://rruff.info/ } \\
\text { Montmorillonite/R110052 }\end{array}$ & $\begin{array}{l}\text { Wahlberg } \\
1962\end{array}$ \\
\hline
\end{tabular}


TABLE 2.3 (Cont.)

\begin{tabular}{|c|c|c|c|c|c|c|c|}
\hline Sorbent Material & $\begin{array}{c}\text { Concentration } \\
\text { of Element }\end{array}$ & $\begin{array}{l}\mathrm{K}_{\mathrm{d}} \text { in } \mathrm{mL} / \mathrm{g} \\
\text { (or other units or } \\
\text { \% sorbed) }\end{array}$ & $\mathrm{CEC}(\mathrm{meq} / 100 \mathrm{~g})$ & $\begin{array}{l}\text { BET Area }\left(\mathrm{m}^{2} / \mathrm{g}\right) \\
\text { or Size (microns) }\end{array}$ & Solution Conditions & Notes & Reference \\
\hline $\begin{array}{l}\text { montmorillonite, No. } 11 \\
\text { (potassium saturated } \\
\text { form, Santa Rita, NM } \\
\text { deposits) }\end{array}$ & $\begin{array}{l}10-10-0.1 \mathrm{~N}+ \\
\text { trace } \mathrm{Cs} 137\end{array}$ & $\begin{array}{l}30,000[\text { low Cs+K] } \\
10[\mathrm{hi} \mathrm{Cs}+\mathrm{K}]\end{array}$ & 116 & $<100$ mesh & $\begin{array}{l}100 \mathrm{mg} \text { clay } / 10 \mathrm{~mL},[\mathrm{~K}]=0.2- \\
0.002 \mathrm{~N}, \mathrm{t}=12 \mathrm{~h}\end{array}$ & $\begin{array}{l}\text { exchg capacity due to isomorphous } \\
\text { substitutions in crystal lattice (per Ref } \\
\text { therein, Lewis-1955, p 54-55) }\end{array}$ & $\begin{array}{l}\text { Wahlberg } \\
1962\end{array}$ \\
\hline $\begin{array}{l}\text { montmorillonite, No. } 11 \\
\text { (Mg saturated form, } \\
\text { Santa Rita, NM } \\
\text { deposits) }\end{array}$ & $\begin{array}{l}10-10-0.1 \mathrm{~N}+ \\
\text { trace } \mathrm{Cs} 137\end{array}$ & $\begin{array}{l}\text { 7,000 [low Cs+Mg] } \\
10[\text { hi Cs+Mg] }\end{array}$ & 116 & $<100$ mesh & $\begin{array}{l}\mathrm{V} / \mathrm{m}=100 \mathrm{~mL} / \mathrm{g}, 100 \mathrm{mg} \text { clay } / \\
10 \mathrm{~mL},[\mathrm{Mg}]=0.2-0.002 \mathrm{~N} \\
\mathrm{t}=12 \mathrm{~h}\end{array}$ & $\begin{array}{l}\text { exchg capacity due to isomorphous } \\
\text { substitutions in crystal lattice (per Ref } \\
\text { therein, Lewis-1955, p 54-55) }\end{array}$ & $\begin{array}{l}\text { Wahlberg } \\
1962\end{array}$ \\
\hline $\begin{array}{l}\text { montmorillonite, No. } 11 \\
\text { (Ca saturated form, } \\
\text { Santa Rita, NM } \\
\text { deposits) }\end{array}$ & $\begin{array}{l}10-10-0.1 \mathrm{~N}+ \\
\text { trace } \mathrm{Cs} 137\end{array}$ & $\begin{array}{l}3,000[\text { low } \mathrm{Cs}+\mathrm{Ca}] \\
10[\mathrm{hi} \mathrm{Cs}+\mathrm{Ca}]\end{array}$ & 116 & $<100$ mesh & $\begin{array}{l}\mathrm{V} / \mathrm{m}=100 \mathrm{~mL} / \mathrm{g}, 100 \mathrm{mg} \\
\text { clay } / 10 \mathrm{~mL},[\mathrm{Ca}]=0.2-0.002 \mathrm{~N}, \\
\mathrm{t}=12 \mathrm{~h}\end{array}$ & $\begin{array}{l}\text { exchg capacity due to isomorphous } \\
\text { substitutions in crystal lattice (per Ref } \\
\text { therein, Lewis-1955, p 54-55) }\end{array}$ & $\begin{array}{l}\text { Wahlberg } \\
1962\end{array}$ \\
\hline $\begin{array}{l}\text { montmorillonite, No. } 21 \\
\text { (sodium saturated form, } \\
\text { Burns, MI deposits) }\end{array}$ & $\begin{array}{l}10-10-0.1 \mathrm{~N}+ \\
\text { trace } \mathrm{Cs} 137\end{array}$ & $\begin{array}{l}20,000[\mathrm{low} \\
\mathrm{Na}+\mathrm{Cs}] \\
10[\mathrm{hi} \mathrm{Na}+\mathrm{Cs}]\end{array}$ & 130 & $<100$ mesh & $\begin{array}{l}\mathrm{V} / \mathrm{m}=100 \mathrm{~mL} / \mathrm{g}, 100 \mathrm{mg} \text { clay } / \\
10 \mathrm{~mL},[\mathrm{Na}]=0.2-0.002 \mathrm{~N} \\
\mathrm{t}=12 \mathrm{~h}\end{array}$ & $\begin{array}{l}\text { exchg capacity due to isomorphous } \\
\text { substitutions in crystal lattice (per Ref } \\
\text { therein, Lewis-1955, p 54-55) }\end{array}$ & $\begin{array}{l}\text { Wahlberg } \\
1962\end{array}$ \\
\hline $\begin{array}{l}\text { montmorillonite, No. } 21 \\
\text { (potassium saturated } \\
\text { form, Burns, MI } \\
\text { deposits) }\end{array}$ & $\begin{array}{l}10-10-0.1 \mathrm{~N}+ \\
\text { trace } \mathrm{Cs} 137\end{array}$ & $\begin{array}{l}30,000[\text { low Cs+K] } \\
10[\text { hi Cs+K] }\end{array}$ & 130 & $<100$ mesh & $\begin{array}{l}100 \mathrm{mg} \text { clay } / 10 \mathrm{~mL},[\mathrm{~K}]=0.2- \\
0.002 \mathrm{~N}, \mathrm{t}=12 \mathrm{~h}\end{array}$ & $\begin{array}{l}\text { exchg capacity due to isomorphous } \\
\text { substitutions in crystal lattice (per Ref } \\
\text { therein, Lewis-1955, p 54-55) }\end{array}$ & $\begin{array}{l}\text { Wahlberg } \\
1962\end{array}$ \\
\hline $\begin{array}{l}\text { montmorillonite, No. } 21 \\
\text { (magnesium saturated } \\
\text { form, Burns, MI } \\
\text { deposits) }\end{array}$ & $\begin{array}{l}10-10-0.1 \mathrm{~N}+ \\
\text { trace } \mathrm{Cs} 137\end{array}$ & $\begin{array}{l}2,000[\text { low } \mathrm{Cs}+\mathrm{Mg}] \\
10[\text { hi } \mathrm{Cs}+\mathrm{Mg}]\end{array}$ & 130 & $<100$ mesh & $\begin{array}{l}\mathrm{V} / \mathrm{m}=100 \mathrm{~mL} / \mathrm{g}, 100 \mathrm{mg} \text { clay } / \\
10 \mathrm{~mL},[\mathrm{Mg}]=0.2-0.002 \mathrm{~N} \\
\mathrm{t}=12 \mathrm{~h}\end{array}$ & $\begin{array}{l}\text { exchg capacity due to isomorphous } \\
\text { substitutions in crystal lattice (per Ref } \\
\text { therein, Lewis-1955, p 54-55) }\end{array}$ & $\begin{array}{l}\text { Wahlberg } \\
1962\end{array}$ \\
\hline $\begin{array}{l}\text { montmorillonite, No. } 21 \\
\text { (calcium saturated form, } \\
\text { Burns, MI deposits) }\end{array}$ & $\begin{array}{l}10-10-0.1 \mathrm{~N}+ \\
\text { trace } \mathrm{Cs} 137\end{array}$ & $\begin{array}{l}3,000[\text { low } \mathrm{Cs}+\mathrm{Ca}] \\
10[\mathrm{hi} \mathrm{Cs}+\mathrm{Ca}]\end{array}$ & 130 & $<100$ mesh & $\begin{array}{l}\mathrm{V} / \mathrm{m}=100 \mathrm{~mL} / \mathrm{g}, 100 \mathrm{mg} \\
\text { clay } / 10 \mathrm{~mL},[\mathrm{Ca}]=0.2-0.002 \mathrm{~N}, \\
\mathrm{t}=12 \mathrm{~h}\end{array}$ & $\begin{array}{l}\text { exchg capacity due to isomorphous } \\
\text { substitutions in crystal lattice (per Ref } \\
\text { therein, Lewis-1955, p 54-55) }\end{array}$ & $\begin{array}{l}\text { Wahlberg } \\
1962\end{array}$ \\
\hline $\begin{array}{l}\text { illite, No. } 35 \text { (sodium } \\
\text { saturated form, Fithian, } \\
\text { IL deposits) }\end{array}$ & $\begin{array}{l}10-10-0.1 \mathrm{~N}+ \\
\text { trace } \mathrm{Cs} 137\end{array}$ & $\begin{array}{l}200,000[\text { low } \\
\mathrm{Cs}+\mathrm{Na}] \\
2[\mathrm{hi} \mathrm{Cs}+\mathrm{Na}]\end{array}$ & 15 & $<100$ mesh & $\begin{array}{l}\mathrm{V} / \mathrm{m}=100 \mathrm{~mL} / \mathrm{g}, 100 \mathrm{mg} \text { clay } / \\
10 \mathrm{~mL},[\mathrm{Na}]=0.2-0.002 \mathrm{~N} \\
\mathrm{t}=12 \mathrm{~h}\end{array}$ & $\begin{array}{l}\text { exchg capacity due to isomorphous } \\
\text { substitutions in the crystal lattice and } \\
\text { to broken bonds at edges of clay } \\
\text { particles (per Ref therein, Grim-1953, } \\
\text { p 132-133) }\end{array}$ & $\begin{array}{l}\text { Wahlberg } \\
1962\end{array}$ \\
\hline
\end{tabular}


TABLE 2.3 (Cont.)

\begin{tabular}{|c|c|c|c|c|c|c|c|}
\hline Sorbent Material & $\begin{array}{c}\text { Concentration } \\
\text { of Element }\end{array}$ & $\begin{array}{l}\mathrm{K}_{\mathrm{d}} \text { in } \mathrm{mL} / \mathrm{g} \\
\text { (or other units or } \\
\% \text { sorbed) }\end{array}$ & $\mathrm{CEC}(\mathrm{meq} / 100 \mathrm{~g})$ & $\begin{array}{l}\text { BET Area }\left(\mathrm{m}^{2} / \mathrm{g} \text { ) }\right. \\
\text { or Size (microns) }\end{array}$ & Solution Conditions & Notes & Reference \\
\hline $\begin{array}{l}\text { illite, No. } 35 \text { (potassium } \\
\text { saturated form, Fithian, } \\
\text { IL deposits) }\end{array}$ & $\begin{array}{l}10-10-0.1 \mathrm{~N}+ \\
\text { trace } \mathrm{Cs} 137\end{array}$ & $\begin{array}{l}2,000[\text { low Cs+K] } \\
3[\mathrm{hi} \mathrm{Cs}+\mathrm{K}]\end{array}$ & 15 & $<100$ mesh & $\begin{array}{l}\mathrm{V} / \mathrm{m}=100 \mathrm{~mL} / \mathrm{g}, 100 \mathrm{mg} \text { clay } / \\
10 \mathrm{~mL},[\mathrm{~K}]=0.2-0.002 \mathrm{~N} \\
\mathrm{t}=12 \mathrm{~h}\end{array}$ & $\begin{array}{l}\text { exchg capacity due to isomorphous } \\
\text { substitutions in the crystal lattice and } \\
\text { to broken bonds at edges of clay } \\
\text { particles (per Ref therein, Grim-1953, } \\
\text { p 132-133) }\end{array}$ & $\begin{array}{l}\text { Wahlberg } \\
1962\end{array}$ \\
\hline $\begin{array}{l}\text { halloysite, No. } 12 \mathrm{~b} \\
\text { (sodium saturated form, } \\
\text { Bedford, IN deposits) }\end{array}$ & $\begin{array}{l}10-10-0.1 \mathrm{~N}+ \\
\text { trace } \mathrm{Cs} 137\end{array}$ & $\begin{array}{l}150,000[\text { low } \\
\mathrm{Cs}+\mathrm{Na}] \\
1[\mathrm{hi} \mathrm{Cs+Na}]\end{array}$ & 19 & $<100$ mesh & $\begin{array}{l}\mathrm{V} / \mathrm{m}=100 \mathrm{~mL} / \mathrm{g}, 100 \mathrm{mg} \text { clay } / \\
10 \mathrm{~mL},[\mathrm{Na}]=0.2-0.002 \mathrm{~N} \\
\mathrm{t}=12 \mathrm{~h}\end{array}$ & $\begin{array}{l}\text { exchg capacity due to dissociation of } \\
\text { structural hydroxyl groups at surface of } \\
\text { crystals and to broken bonds at edges } \\
\text { of clay particles (per Ref therein, } \\
\text { Grim-1953) }\end{array}$ & $\begin{array}{l}\text { Wahlberg } \\
1962\end{array}$ \\
\hline $\begin{array}{l}\text { halloysite, No. 12b } \\
\text { (potassium saturated } \\
\text { form, Bedford, IN } \\
\text { deposits) }\end{array}$ & $\begin{array}{l}10-10-0.1 \mathrm{~N}+ \\
\text { trace } \mathrm{Cs} 137\end{array}$ & $\begin{array}{l}400[\text { low Cs+K] } \\
1[\mathrm{hi} \mathrm{Cs}+\mathrm{K}]\end{array}$ & 19 & $<100$ mesh & $\begin{array}{l}\mathrm{V} / \mathrm{m}=100 \mathrm{~mL} / \mathrm{g}, 100 \mathrm{mg} \text { clay } / \\
10 \mathrm{~mL},[\mathrm{~K}]=0.2-0.002 \mathrm{~N} \\
\mathrm{t}=12 \mathrm{~h}\end{array}$ & $\begin{array}{l}\text { exchg capacity due to dissociation of } \\
\text { structural hydroxyl groups at surface of } \\
\text { crystals and to broken bonds at edges } \\
\text { of clay particles (per Ref therein, } \\
\text { Grim-1953) }\end{array}$ & $\begin{array}{l}\text { Wahlberg } \\
1962\end{array}$ \\
\hline $\begin{array}{l}\text { halloysite, No. 12b } \\
\text { (magnesium saturated } \\
\text { form, Bedford, IN } \\
\text { deposits) }\end{array}$ & $\begin{array}{l}10-10-0.1 \mathrm{~N}+ \\
\text { trace } \mathrm{Cs} 137\end{array}$ & $\begin{array}{l}40,000[\text { low } \\
\mathrm{Cs}+\mathrm{Mg}] \\
3[\mathrm{hi} \mathrm{Cs}+\mathrm{Mg}]\end{array}$ & 19 & $<100$ mesh & $\begin{array}{l}\mathrm{V} / \mathrm{m}=100 \mathrm{~mL} / \mathrm{g}, 100 \mathrm{mg} \text { clay } / \\
10 \mathrm{~mL},[\mathrm{Mg}]=0.2-0.002 \mathrm{~N} \\
\mathrm{t}=12 \mathrm{~h}\end{array}$ & $\begin{array}{l}\text { exchg capacity due to dissociation of } \\
\text { structural hydroxyl groups at surface of } \\
\text { crystals and to broken bonds at edges } \\
\text { of clay particles (per Ref therein, } \\
\text { Grim-1953) }\end{array}$ & $\begin{array}{l}\text { Wahlberg } \\
1962\end{array}$ \\
\hline $\begin{array}{l}\text { halloysite, No. } 12 \mathrm{~b} \\
\text { (calcium saturaed form, } \\
\text { Bedford, IN deposits) }\end{array}$ & $\begin{array}{l}10-10-0.1 \mathrm{~N}+ \\
\text { trace } \mathrm{Cs} 137\end{array}$ & $\begin{array}{l}30,000[\text { low } \\
\text { Cs+Ca] } \\
1[\mathrm{hi} \mathrm{Cs}+\mathrm{Ca}]\end{array}$ & 19 & $<100$ mesh & $\begin{array}{l}\mathrm{V} / \mathrm{m}=100 \mathrm{~mL} / \mathrm{g}, 100 \mathrm{mg} \text { clay } / \\
10 \mathrm{~mL},[\mathrm{Ca}]=0.2-0.002 \mathrm{~N} \\
\mathrm{t}=12 \mathrm{~h}\end{array}$ & $\begin{array}{l}\text { exchg capacity due to dissociation of } \\
\text { structural hydroxyl groups at surface of } \\
\text { crystals and to broken bonds at edges } \\
\text { of clay particles (per Ref therein, } \\
\text { Grim-1953) }\end{array}$ & $\begin{array}{l}\text { Wahlberg } \\
1962\end{array}$ \\
\hline $\begin{array}{l}\text { kaolinite, No. } 4 \text { (sodium } \\
\text { saturated form, Macon, } \\
\text { BA deposits) }\end{array}$ & $\begin{array}{l}10-10-0.1 \mathrm{~N}+ \\
\text { trace Cs137 }\end{array}$ & $\begin{array}{l}700 \text { [low Cs+Na] } \\
1[\mathrm{hi} \mathrm{Cs+Na}]\end{array}$ & 10 & $<100$ mesh & $\begin{array}{l}\mathrm{V} / \mathrm{m}=100 \mathrm{~mL} / \mathrm{g}, 100 \mathrm{mg} \text { clay } / \\
10 \mathrm{~mL},[\mathrm{Na}]=0.2-0.002 \mathrm{~N} \\
\mathrm{t}=12 \mathrm{~h}\end{array}$ & $\begin{array}{l}\text { exchg capacity due to dissociation of } \\
\text { structural hydroxyl groups at surface of } \\
\text { crystals and to broken bonds at edges } \\
\text { of clay particles (per Ref therein, } \\
\text { Grim-1953) }\end{array}$ & $\begin{array}{l}\text { Wahlberg } \\
1962\end{array}$ \\
\hline
\end{tabular}


TABLE 2.3 (Cont.)

\begin{tabular}{|c|c|c|c|c|c|c|c|}
\hline Sorbent Material & $\begin{array}{c}\text { Concentration } \\
\text { of Element }\end{array}$ & $\begin{array}{c}\mathrm{K}_{\mathrm{d}} \text { in } \mathrm{mL} / \mathrm{g} \\
\text { (or other units or } \\
\% \text { sorbed) }\end{array}$ & $\mathrm{CEC}(\mathrm{meq} / 100 \mathrm{~g})$ & $\begin{array}{l}\text { BET Area }\left(\mathrm{m}^{2} / \mathrm{g}\right) \\
\text { or Size (microns) }\end{array}$ & Solution Conditions & Notes & Reference \\
\hline $\begin{array}{l}\text { kaolinite, No. } 4 \\
\text { (potassium saturated } \\
\text { form, Macon, BA } \\
\text { deposits) }\end{array}$ & $\begin{array}{l}10-10-0.1 \mathrm{~N}+ \\
\text { trace } \mathrm{Cs} 137\end{array}$ & $\begin{array}{l}700[\text { low } \mathrm{Cs}+\mathrm{K}] \\
1[\mathrm{hi} \mathrm{Cs}+\mathrm{K}]\end{array}$ & 10 & $<100$ mesh & $\begin{array}{l}\mathrm{V} / \mathrm{m}=100 \mathrm{~mL} / \mathrm{g}, 100 \mathrm{mg} \text { clay } / \\
10 \mathrm{~mL},[\mathrm{~K}]=0.2-0.002 \mathrm{~N} \\
\mathrm{t}=12 \mathrm{~h}\end{array}$ & $\begin{array}{l}\text { exchg capacity due to dissociation of } \\
\text { structural hydroxyl groups at surface of } \\
\text { crystals and to broken bonds at edges } \\
\text { of clay particles (per Ref therein, } \\
\text { Grim-1953) }\end{array}$ & $\begin{array}{l}\text { Wahlberg } \\
1962\end{array}$ \\
\hline $\begin{array}{l}\text { kaolinite, No. } 4 \\
\text { (magnesium saturated } \\
\text { form, Macon, BA } \\
\text { deposits) }\end{array}$ & $\begin{array}{l}10-10-0.1 \mathrm{~N}+ \\
\text { trace } \mathrm{Cs} 137\end{array}$ & $\begin{array}{l}400[\text { low } \mathrm{Cs}+\mathrm{Mg}] \\
2[\mathrm{hi} \mathrm{Cs}+\mathrm{Mg}]\end{array}$ & 10 & $<100$ mesh & $\begin{array}{l}\mathrm{V} / \mathrm{m}=100 \mathrm{~mL} / \mathrm{g}, 100 \mathrm{mg} \text { clay } / \\
10 \mathrm{~mL}],[\mathrm{Mg}]=0.2-0.002 \mathrm{~N} \\
\mathrm{t}=12 \mathrm{~h}\end{array}$ & $\begin{array}{l}\text { exchg capacity due to dissociation of } \\
\text { structural hydroxyl groups at surface of } \\
\text { crystals and to broken bonds at edges } \\
\text { of clay particles (per Ref therein, } \\
\text { Grim-1953) }\end{array}$ & $\begin{array}{l}\text { Wahlberg } \\
1962\end{array}$ \\
\hline $\begin{array}{l}\text { kaolinite, No. } 4 \text { (calium } \\
\text { saturated form, Macon, } \\
\text { BA deposits) }\end{array}$ & $\begin{array}{l}10-10-0.1 \mathrm{~N}+ \\
\text { trace Cs } 137\end{array}$ & $\begin{array}{l}400[\text { low } \mathrm{Cs}+\mathrm{Ca}] \\
1[\mathrm{hi} \mathrm{Cs}+\mathrm{Ca}]\end{array}$ & 10 & $<100$ mesh & $\begin{array}{l}\mathrm{V} / \mathrm{m}=100 \mathrm{~mL} / \mathrm{g}, 100 \mathrm{mg} \text { clay } / \\
10 \mathrm{~mL},[\mathrm{Ca}]=0.2-0.002 \mathrm{~N} \\
\mathrm{t}=12 \mathrm{~h}\end{array}$ & $\begin{array}{l}\text { exchg capacity due to dissociation of } \\
\text { structural hydroxyl groups at surface of } \\
\text { crystals and to broken bonds at edges } \\
\text { of clay particles (per Ref therein, } \\
\text { Grim-1953) }\end{array}$ & $\begin{array}{l}\text { Wahlberg } \\
1962\end{array}$ \\
\hline illite & $\begin{array}{l}\text { details in } \\
\text { patent? }\end{array}$ & details in patent? & & & details in patent? & $\begin{array}{l}\text { Cesium uptake by plants depends on } \\
\text { adsorption/desorption reactions in the } \\
\text { soil, as well as root uptake processes } \\
\text { controlled by the plant. In this study, } \\
\text { sorption and desorption of Cs+ on ref. } \\
\text { illite (IMt-1) was investigated in the } \\
\text { presence of oxalate to gain } \\
\text { understanding of mechanisms by } \\
\text { which plant root exudates may } \\
\text { influence Cs+ bioavailability in } \\
\text { micaceous soils. Cesium sorption on } \\
\text { illite decreased significantly as oxalate } \\
\text { concn. increased from } 0.4 \text { to } 2 \text { mM. } \\
\text { Cesium desorption from illite increased } \\
\text { significantly with increasing oxalate } \\
\text { concn. Desorption of Cs+ by exchange } \\
\text { with Na+, Ca2+ and Mg2+ was } \\
\text { significantly enhanced in the presence } \\
\text { of oxalate as selectivity for Cs+ }\end{array}$ & $\begin{array}{l}\text { Wendling } \\
2004\end{array}$ \\
\hline
\end{tabular}


TABLE 2.3 (Cont.)

\begin{tabular}{|c|c|c|c|c|c|c|c|}
\hline$-\cdots$ & $\begin{array}{c}\text { Concentration } \\
\text { of Element }\end{array}$ & $\begin{array}{l}\mathrm{K}_{\mathrm{d}} \text { in } \mathrm{mL} / \mathrm{g} \\
\text { (or other units or } \\
\% \text { sorbed) }\end{array}$ & $\mathrm{CEC}(\mathrm{meq} / 100 \mathrm{~g})$ & $\begin{array}{l}\text { BET Area }\left(\mathrm{m}^{2} / \mathrm{g} \text { ) }\right. \\
\text { or Size (microns) }\end{array}$ & Solution Conditions & Notes & Reference \\
\hline Illite (Cont.) & & & & & & $\begin{array}{l}\text { decreased with respect to these ions in } \\
\text { the presence of oxalate. On the other } \\
\text { hand, oxalate had little effect on the } \\
\text { Cs+/K+ selectivity coeff. This suggests } \\
\text { that oxalate treatments increase the } \\
\text { relative proportion of exchange sites } \\
\text { that are not highly selective for Cs+ } \\
\text { and K+; e.g. "planar" sites. The results } \\
\text { indicate that oxalate plays an important } \\
\text { role in Cs+ binding on illite and, } \\
\text { therefore, plant rhizosphere chem. is } \\
\text { likely to alter Cs+ bioavailability in } \\
\text { micaceous soils. }\end{array}$ & \\
\hline $\begin{array}{l}\text { titanium silicate } \\
\text { (synthesized using } \\
\text { TiOSO4) }\end{array}$ & $\begin{array}{l}2 \mathrm{e}-10 \text { and } \\
7 \mathrm{e}-5 \mathrm{M}\end{array}$ & 6 to $4 \mathrm{e} 4 \mathrm{~mL} / \mathrm{g}$ & & 120 to $726 \mathrm{~m} 2 / \mathrm{g}$ & $\begin{array}{l}\mathrm{I}=0.1 \mathrm{M} \mathrm{Na}+, \mathrm{t}=2 \mathrm{~h}, 1 / 100 \text { to } \\
1 / 1000 \mathrm{~g} / \mathrm{mL}\end{array}$ & $\begin{array}{l}\text { Ref therein } 20,21 \mathrm{Cs} \text { and } \mathrm{Sr} \text { sorption } \\
\text { onto crystalline } \mathrm{TiSi}\end{array}$ & $\begin{array}{l}\text { Lujaniene } \\
2009\end{array}$ \\
\hline $\begin{array}{l}\text { chazabite, clinoptilolite, } \\
\text { HTiSi, KTiGe, } \\
\text { KTiSiGe, KTSgel, } \\
\text { KTSsol, NaTi }\end{array}$ & trace Cs-137? & $1 \mathrm{e} 3$ to $2 \mathrm{e} 5 \mathrm{~mL} / \mathrm{g}$ & & & $\begin{array}{l}\mathrm{pH}=11,1: 200 \text { and } \\
1: 1000 \mathrm{~g} / \mathrm{mL}, \text { groundwater } \\
\text { simulant: }[\mathrm{Na}]=3 \mathrm{e}-4 \mathrm{M}, \\
{[\mathrm{Mg}]=2 \mathrm{e}-4 \mathrm{M},[\mathrm{CO} 32-]=1 \mathrm{e}-} \\
4 \mathrm{M},[\mathrm{OH}-]=2 \mathrm{e}-3 \mathrm{M},[\mathrm{NO} 3- \\
]=2 \mathrm{e}-4 \mathrm{M},[\mathrm{SO} 42-]=2 \mathrm{e}-4 \mathrm{M}\end{array}$ & $\begin{array}{l}\text { Table } 3 \text { summarizes Kd_Cs for } \\
\text { sorbents, KTiSiGe }>\text { KTiGe } \sim \text { HTiSi } \sim \\
\text { KTSsol, lowest w NaTi }\end{array}$ & $\begin{array}{l}\text { Behrens } \\
1998\end{array}$ \\
\hline $\begin{array}{l}\text { chazabite, clinoptilolite, } \\
\text { HTiSi, KTiGe, } \\
\text { KTiSiGe, KTSgel, } \\
\text { KTSsol, NaTi }\end{array}$ & trace & 7 to $300 \mathrm{~mL} / \mathrm{g}$ & & & $\begin{array}{l}\mathrm{pH}=14,1: 200 \mathrm{~g} / \mathrm{mL}, \text { defense } \\
\text { waste simulants: }[\mathrm{Na}]=5 \mathrm{M}, \\
{[\mathrm{Al}]=0.4 \mathrm{M},[\mathrm{K}]=0.03-0.1,} \\
{[\mathrm{CO} 32-]=0.04-0.2 \mathrm{M},} \\
{[\mathrm{OH}-]=2 \mathrm{M},[\mathrm{NO} 3-]=2 \mathrm{M}}\end{array}$ & $\begin{array}{l}\text { Tables } 4 \text { and } 5 \text { summarizes Kd_Cs for } \\
\text { sorbents, NaTi > HTiSi > KTSgel }\end{array}$ & $\begin{array}{l}\text { Behrens } \\
1998\end{array}$ \\
\hline $\begin{array}{l}\text { crystalline silicotitanate } \\
\text { (CST, Ionsiv IE-910, } \\
\text { manufactured by UOP) }\end{array}$ & $6 \mathrm{ug} / \mathrm{L} \mathrm{Cs}-137$ & $5 \mathrm{e} 3$ to $9 \mathrm{e} 4 \mathrm{~mL} / \mathrm{g}$ & & & $\begin{array}{l}\mathrm{pH}=12-13, \mathrm{t}=0.5,2,6 \mathrm{~h}, \\
{[\mathrm{Na}]=2 \mathrm{M},[\mathrm{Al}]=0.01-0.2,} \\
{[\mathrm{CO} 32-]=0.2-0.6 \mathrm{M},} \\
{[\mathrm{TOC}]=0.1-1 \mathrm{M}, \text { four } 3: 1} \\
\text { diluted Hanford complexant } \\
\text { conc simulant solutions } \\
\text { (Table } 1 \text { for additional minor } \\
\text { components) }\end{array}$ & $\begin{array}{l}\text { Table } 6 \text { includes time dependency of } \\
\text { Kd's for various solutions; lower Kds } \\
\text { with organic complexants (untreated } \\
\text { solutions) }\end{array}$ & Marsh 1995 \\
\hline
\end{tabular}


TABLE 2.3 (Cont.)

\begin{tabular}{|c|c|c|c|c|c|c|c|}
\hline Sorbent Material & $\begin{array}{c}\text { Concentration } \\
\text { of Element }\end{array}$ & $\begin{array}{c}\mathrm{K}_{\mathrm{d}} \text { in } \mathrm{mL} / \mathrm{g} \\
\text { (or other units or } \\
\% \text { sorbed) }\end{array}$ & $\mathrm{CEC}(\mathrm{meq} / 100 \mathrm{~g})$ & $\begin{array}{l}\text { BET Area }\left(\mathrm{m}^{2} / \mathrm{g} \text { ) }\right. \\
\text { or Size (microns) }\end{array}$ & Solution Conditions & Notes & Reference \\
\hline $\begin{array}{l}\text { potassium } \\
\text { hexacyanoferrate } \\
\text { crystals (KCoFC, prepd } \\
\text { at ORNL) and } \\
\text { composite (KCoFC- } \\
\text { PAN, prepd by F. } \\
\text { Sebesta, Czech Techn. } \\
\text { Univ.) }\end{array}$ & $6 \mathrm{ug} / \mathrm{L} \mathrm{Cs}-137$ & $2 \mathrm{e} 2$ to $9 \mathrm{e} 4 \mathrm{~mL} / \mathrm{g}$ & & $\begin{array}{l}150-600 \text { micron } \\
\text { crystals }\end{array}$ & $\begin{array}{l}\mathrm{pH}=12-13, \mathrm{t}=0.5,2,6 \mathrm{~h}, \\
{[\mathrm{Na}]=2 \mathrm{M},[\mathrm{Al}]=0.01-0.2,} \\
{[\mathrm{CO} 2-]=0.2-0.6 \mathrm{M},} \\
{[\mathrm{TOC}]=0.1-1 \mathrm{M}, \text { four } 3: 1} \\
\text { diluted Hanford complexant } \\
\text { conc simulant solutions } \\
\text { (Table } 1 \text { for additional minor } \\
\text { components) }\end{array}$ & $\begin{array}{l}\text { Table } 6 \text { includes time dependency of } \\
\text { Kd's for various solutions; lower Kds } \\
\text { with organic complexants (untreated } \\
\text { solutions) }\end{array}$ & Marsh 1995 \\
\hline $\begin{array}{l}\text { nickel hexacyanoferrate } \\
\text { composite (NiFC-PAN, } \\
\text { prepd by F. Sebesta, } \\
\text { Czech Techn. Univ.) }\end{array}$ & 6 ug/L Cs-137 & $2 \mathrm{e} 2$ to $9 \mathrm{e} 4 \mathrm{~mL} / \mathrm{g}$ & & & $\begin{array}{l}\mathrm{pH}=12-13, \mathrm{t}=0.5,2,6 \mathrm{~h}, \\
{[\mathrm{Na}]=2 \mathrm{M},[\mathrm{Al}]=0.01-0.2,} \\
{[\mathrm{CO} 32-]=0.2-0.6 \mathrm{M},} \\
{[\mathrm{TOC}]=0.1-1 \mathrm{M}, \text { four } 3: 1} \\
\text { diluted Hanford complexant } \\
\text { conc simulant solutions } \\
\text { (Table } 1 \text { for additional minor } \\
\text { components) }\end{array}$ & $\begin{array}{l}\text { Table } 6 \text { includes time dependency of } \\
\text { Kd's for various solutions; lower Kds } \\
\text { with organic complexants (untreated } \\
\text { solutions) }\end{array}$ & Marsh 1995 \\
\hline $\begin{array}{l}\text { K-Ni hexacyanoferrate } \\
\text { (KNiFC-CsTreat prepd } \\
\text { by Japan) }\end{array}$ & 10ppm Cs-134 & $\begin{array}{l}\text { 1e4-1e6 depending } \\
\text { on solution }\end{array}$ & $\begin{array}{l}2.58 \mathrm{meq} / \mathrm{g} \mathrm{K} \text { and } \\
3.34 \mathrm{meg} / \mathrm{g} \mathrm{Ni}\end{array}$ & $\begin{array}{l}<100 \text { mesh } \\
(<149 \text { um })\end{array}$ & $\begin{array}{l}10 \mathrm{mg} / \mathrm{mL} \text {. Effect of } \mathrm{pH} \text {, } \\
\mathrm{Na}+, \mathrm{K}+, \mathrm{NH} 4+, \mathrm{Ca} 2+\text { ions, } \\
\text { particle size, kinetics. } \\
\text { Samples equilibrated for } 5 \mathrm{~d} \\
\text { for tests. }\end{array}$ & $\begin{array}{l}\text { Uptake kinetics for smaller than } 100 \\
\text { mesh was at equilibrium by } 1 \mathrm{st} \text { time pt } \\
(<<1 \text { dy). Kd insensitive to } \mathrm{Na} \text { or } \mathrm{Ca} \\
\text { and increases with } \mathrm{pH} \text {, but drops } \\
\text { gradually with } \mathrm{K}+(\mathrm{Kd}=50,000 \text { at } 1 \\
\mathrm{M}) \text {. Drop is linear above } \sim 0.1 \mathrm{M} \mathrm{NH} 4+ \\
\text { but still } 7-8000 \mathrm{ml} / \mathrm{g} \text { at } 1 \mathrm{M} \mathrm{NH} 4+\text {. }\end{array}$ & $\begin{array}{l}\text { Mimura } \\
1997\end{array}$ \\
\hline $\begin{array}{l}\text { resorcinol/formaldehyde } \\
\text { resin (BSC-210, } \\
\text { manufactured by } \\
\text { Boulder Sci. Co., } \\
\text { converted to Na form) }\end{array}$ & 6 ug/L Cs-137 & $1 \mathrm{e} 3$ to $7 \mathrm{e} 3 \mathrm{~mL} / \mathrm{g}$ & & & $\begin{array}{l}\mathrm{pH}=12-13, \mathrm{t}=0.5,2,6 \mathrm{~h}, \\
{[\mathrm{Na}]=2 \mathrm{M},[\mathrm{Al}]=0.01-0.2,} \\
{[\mathrm{CO} 32-]=0.2-0.6 \mathrm{M},} \\
{[\mathrm{TOC}]=0.1-1 \mathrm{M}, \text { four } 3: 1} \\
\text { diluted Hanford complexant } \\
\text { conc simulant solutions } \\
\text { (Table } 1 \text { for additional minor } \\
\text { components) }\end{array}$ & $\begin{array}{l}\text { Table } 6 \text { includes time dependency of } \\
\text { Kd's for various solutions; lower Kds } \\
\text { with organic complexants (untreated } \\
\text { solutions) }\end{array}$ & Marsh 1995 \\
\hline
\end{tabular}


TABLE 2.3 (Cont.)

\begin{tabular}{|c|c|c|c|c|c|c|c|}
\hline Sorbent Material & $\begin{array}{c}\text { Concentration } \\
\text { of Element }\end{array}$ & $\begin{array}{l}\mathrm{K}_{\mathrm{d}} \text { in } \mathrm{mL} / \mathrm{g} \\
\text { (or other units or } \\
\% \text { sorbed) }\end{array}$ & CEC (meq/100g) & $\begin{array}{l}\text { BET Area }\left(\mathrm{m}^{2} / \mathrm{g} \text { ) }\right. \\
\text { or Size (microns) }\end{array}$ & Solution Conditions & Notes & Reference \\
\hline $\begin{array}{l}\text { Superlig } 666 \\
\text { (proprietary macrocycle, } \\
\text { prepd by IBC Adv. } \\
\text { Technol., Inc.) }\end{array}$ & 6 ug/L Cs-137 & 30 to $4 \mathrm{e} 3 \mathrm{~mL} / \mathrm{g}$ & & & $\begin{array}{l}\mathrm{pH}=12-13, \mathrm{t}=0.5,2,6 \mathrm{~h}, \\
{[\mathrm{Na}]=2 \mathrm{M},[\mathrm{Al}]=0.01-0.2,} \\
{[\mathrm{CO} 32-]=0.2-0.6 \mathrm{M},} \\
{[\mathrm{TOC}]=0.1-1 \mathrm{M}, \text { four } 3: 1} \\
\text { diluted Hanford complexant } \\
\text { conc simulant solutions } \\
\text { (Table } 1 \text { for additional minor } \\
\text { components) }\end{array}$ & $\begin{array}{l}\text { Table } 6 \text { includes time dependency of } \\
\text { Kd's for various solutions; higher Kds } \\
\text { with organic complexants (untreated } \\
\text { solutions) }\end{array}$ & Marsh 1995 \\
\hline $\begin{array}{l}\text { SuperLig } 644 \\
\text { (polymeric resin } \\
\text { containing poly } \\
\text { (hydroxyarlyne) groups) } \\
\text { ligand }\end{array}$ & $\begin{array}{l}\mathrm{Cs} 137=\sim 200 \\
\mathrm{uCi} / \mathrm{mL} \\
6.7 \times 10^{\wedge}-5 \mathrm{M} \\
\text { total Cs }\end{array}$ & $\begin{array}{l}400-2000 \mathrm{~mL} / \mathrm{g} \\
\text { depending on } \\
\mathrm{Na} / \mathrm{Cs} \text { ratio. }\end{array}$ & $\begin{array}{l}0.72 \text { to } 3.46 \\
\text { mmole/g resin, } \\
\text { depending on the } \\
\text { Hanford tank } \\
\text { composition }\end{array}$ & $\begin{array}{l}20-70 \text { mesh } \\
\text { granules and was } \\
\text { used received }\end{array}$ & $\begin{array}{l}\text { General composition was } 5 \mathrm{M} \\
\mathrm{Na}+\mathrm{K}(0.12 \mathrm{M}), \mathrm{Al}(0.7), \\
\mathrm{NO} 3-(1.0 \mathrm{M}), \mathrm{NO} 2-(0.87 \mathrm{M}), \\
\text { and free } \mathrm{OH}-(1.9 \mathrm{M}) \text { but } \\
\text { varied between tank } \\
\text { solutions. }\end{array}$ & $\begin{array}{l}\text { volume/resin mass ratio (phase ratio) } \\
\text { was typically } \sim 100: 1 \text {. }\end{array}$ & $\begin{array}{l}\text { Hassan } \\
2002\end{array}$ \\
\hline $\begin{array}{l}\text { potassium } \\
\text { hexacyanoferrate } \\
\text { crystals (KCoFC, prepd } \\
\text { at ORNL) }\end{array}$ & 6 ug/L Cs-137 & $7 \mathrm{e} 1$ to $9 \mathrm{e} 4 \mathrm{~mL} / \mathrm{g}$ & & $150-600$ microns & $\begin{array}{l}\mathrm{pH}=14, \mathrm{t}=0.5,2,6 \mathrm{~h}, \\
{[\mathrm{Na}]=4 \mathrm{M},[\mathrm{Al}]=0.4 \mathrm{M},} \\
{[\mathrm{CO} 32-]=2 \mathrm{M},[\mathrm{TOC}]=0.1-} \\
0.7 \mathrm{M}, \text { four Hanford Tank } \\
\text { 101-SY simulant solutions } \\
\text { (Table } 1 \text { for additional minor } \\
\text { components) }\end{array}$ & $\begin{array}{l}\text { Table } 6 \text { includes time dependency of } \\
\text { Kd's for various solutions; lower Kds } \\
\text { with organic complexants (untreated } \\
\text { solutions) }\end{array}$ & Marsh 1995 \\
\hline $\begin{array}{l}\text { nickel hexacyanoferrate } \\
\text { composite (NiFC-PAN, } \\
\text { prepd by F. Sebesta, } \\
\text { Czech Techn. Univ.) }\end{array}$ & 6 ug/L Cs-137 & $3 \mathrm{e} 1$ to $3 \mathrm{e} 3 \mathrm{~mL} / \mathrm{g}$ & & & $\begin{array}{l}\mathrm{pH}=14, \mathrm{t}=0.5,2,6 \mathrm{~h}, \\
{[\mathrm{Na}]=4 \mathrm{M},[\mathrm{Al}]=0.4 \mathrm{M},} \\
{[\mathrm{CO} 32-]=2 \mathrm{M},[\mathrm{TOC}]=0.1-} \\
0.7 \mathrm{M}, \text { four Hanford Tank } \\
101-\mathrm{SY} \text { simulant solutions } \\
\text { (Table } 1 \text { for additional minor } \\
\text { components) }\end{array}$ & $\begin{array}{l}\text { Table } 6 \text { includes time dependency of } \\
\text { Kd's for various solutions; lower Kds } \\
\text { with organic complexants (untreated } \\
\text { solutions) }\end{array}$ & Marsh 1995 \\
\hline
\end{tabular}


TABLE 2.3 (Cont.)

\begin{tabular}{|c|c|c|c|c|c|c|c|}
\hline Sorbent Material & $\begin{array}{c}\text { Concentration } \\
\text { of Element }\end{array}$ & $\begin{array}{l}\mathrm{K}_{\mathrm{d}} \text { in } \mathrm{mL} / \mathrm{g} \\
\text { (or other units or } \\
\% \text { sorbed) }\end{array}$ & $\mathrm{CEC}(\mathrm{meq} / 100 \mathrm{~g})$ & $\begin{array}{l}\text { BET Area }\left(\mathrm{m}^{2} / \mathrm{g}\right) \\
\text { or Size (microns) }\end{array}$ & Solution Conditions & Notes & Reference \\
\hline $\begin{array}{l}\text { Ammonium } \\
\text { molybdophosphate- } \\
\text { polyacrylonitrile (AMP- } \\
\text { PAN) composite } \\
\text { sorbents (prepd by F. } \\
\text { Sebesta, Czech Techn. } \\
\text { Univ.) }\end{array}$ & $\begin{array}{l}100 \mathrm{mg} \text { AMP- } \\
\text { PAN in } \\
15 \mathrm{mg} / \mathrm{L} \mathrm{Cs} \text { in } \\
10 \mathrm{~mL} \text { solution. }\end{array}$ & $\begin{array}{l}>10^{\wedge} 5 \mathrm{~mL} / \mathrm{g} \text { in } 1-2 \\
\mathrm{HNO} 3 \mathrm{and} / \mathrm{or} 1-2 \mathrm{M} \\
\mathrm{NaNO} 3 ; 4000 \mathrm{~mL} / \mathrm{g} \\
\text { in } 0.5 \mathrm{M} \mathrm{KNO} 3 \text { and } \\
2000 \mathrm{~mL} / \mathrm{g} \text { in } 1 \mathrm{M} \\
\mathrm{KNO} 3 \text { in } 1- \\
2 \mathrm{MHNO} 3\end{array}$ & $\begin{array}{l}22.5,19.8 \text { and } 19.6 \\
\mathrm{mg} \mathrm{Cs} / \mathrm{g} \text { sorbent for } \\
5,10 \text { and } 20 \mathrm{BV} / \mathrm{hr}\end{array}$ & $\begin{array}{l}0.3-0.6 \mathrm{~mm} \\
\text { diameter }\end{array}$ & & $\begin{array}{l}\text { AMP-PAN/SF02 sorbent was } \\
\text { composed of } 85.7 \mathrm{wt} \% \text { AMP and } 14.3 \\
\text { wt } \% \text { PAN. } 1.5 \mathrm{~cm} 3 \text { column BV at } \\
\text { flowrates of } 5,10 \text { and } 20 \text { bed volumes } \\
\text { per hour }\end{array}$ & \\
\hline $\begin{array}{l}\text { crystalline silicotitanate } \\
\text { (Ionsiv IE-910, CST, } \\
\text { manufactured by UOP) }\end{array}$ & $6 \mathrm{ug} / \mathrm{L} \mathrm{Cs}-137$ & $2 \mathrm{e} 3$ to $4 \mathrm{e} 3 \mathrm{~mL} / \mathrm{g}$ & & & $\begin{array}{l}\mathrm{pH}=14, \mathrm{t}=0.5,2,6 \mathrm{~h}, \\
{[\mathrm{Na}]=4 \mathrm{M},[\mathrm{Al}]=0.4 \mathrm{M},} \\
{[\mathrm{CO} 32-]=2 \mathrm{M},[\mathrm{TOC}]=0.1-} \\
0.7 \mathrm{M}, \text { four Hanford Tank } \\
\text { 101-SY simulant solutions } \\
\text { (Table } 1 \text { for additional minor } \\
\text { components) }\end{array}$ & $\begin{array}{l}\text { Table } 6 \text { includes time dependency of } \\
\text { Kd's for various solutions; slightly } \\
\text { lower Kds with organic complexants } \\
\text { (untreated solutions) }\end{array}$ & Marsh 1995 \\
\hline $\begin{array}{l}\text { resorcinol/formaldehyde } \\
\text { resin (BSC-210, } \\
\text { manufactured by } \\
\text { Boulder Sci. Co., } \\
\text { converted to Na form) }\end{array}$ & $6 \mathrm{ug} / \mathrm{L}$ Cs-137 & $6 \mathrm{e} 2$ to $3 \mathrm{e} 3 \mathrm{~mL} / \mathrm{g}$ & & & $\begin{array}{l}\mathrm{pH}=14, \mathrm{t}=0.5,2,6 \mathrm{~h}, \\
{[\mathrm{Na}]=4 \mathrm{M},[\mathrm{Al}]=0.4 \mathrm{M},} \\
{[\mathrm{CO} 2-]=2 \mathrm{M},[\mathrm{TOC}]=0.1-} \\
0.7 \mathrm{M}, \text { four Hanford Tank } \\
\text { 101-SY simulant solutions } \\
\text { (Table } 1 \text { for additional minor } \\
\text { components) }\end{array}$ & $\begin{array}{l}\text { Table } 6 \text { includes time dependency of } \\
\text { Kd's for various solutions; slightly } \\
\text { lower Kds with organic complexants } \\
\text { (untreated solutions) }\end{array}$ & Marsh 1995 \\
\hline $\begin{array}{l}\text { polymeric macrocycle } \\
\text { (Superlig } 666, \\
\text { proprietary, prepd by } \\
\text { IBC Adv. Technol., Inc.) }\end{array}$ & 6 ug/L Cs-137 & $6 \mathrm{e} 2$ to $4 \mathrm{e} 3 \mathrm{~mL} / \mathrm{g}$ & & & $\begin{array}{l}\mathrm{pH}=14, \mathrm{t}=0.5,2,6 \mathrm{~h}, \\
{[\mathrm{Na}]=4 \mathrm{M},[\mathrm{Al}]=0.4 \mathrm{M},} \\
{[\mathrm{CO} 2-]=2 \mathrm{M},[\mathrm{TOC}]=0.1-} \\
0.7 \mathrm{M}, \text { four Hanford Tank } \\
\text { 101-SY simulant solutions } \\
\text { (Table } 1 \text { for additional minor } \\
\text { components) }\end{array}$ & $\begin{array}{l}\text { Table } 6 \text { includes time dependency of } \\
\text { Kd's for various solutions; slightly } \\
\text { lower Kds with organic complexants } \\
\text { (untreated solutions) }\end{array}$ & Marsh 1995 \\
\hline $\begin{array}{l}\text { silicotitanate (IONSIV } \\
\text { IE-911, UOP) }\end{array}$ & trace Cs-137 & $710 \mathrm{~mL} / \mathrm{g}$ & & & $\begin{array}{l}\mathrm{pH}>14, \mathrm{t}=72 \mathrm{~h},[\mathrm{Na}]=5 \mathrm{M}, \\
{[\mathrm{K}]=0.5 \mathrm{M}, \mathrm{Na} / \mathrm{Cs}=8 \mathrm{e} 4} \\
\text { (Attachment } \mathrm{A} \text { for detailed } \\
\text { contents of DSSF waste } \\
\text { simulant) }\end{array}$ & $\begin{array}{l}12-14 \% \text { increase in Cs loading with } \\
25 \text {-fold dilution of waste ( } 5 \mathrm{M} \text { to } 0.2 \mathrm{M} \\
\mathrm{Na} \text { ) }\end{array}$ & Brown 1996 \\
\hline
\end{tabular}


TABLE 2.3 (Cont.)

\begin{tabular}{|c|c|c|c|c|c|c|c|}
\hline$\cdots$ & $\begin{array}{c}\text { Concentration } \\
\text { of Element }\end{array}$ & $\begin{array}{l}\mathrm{K}_{\mathrm{d}} \text { in } \mathrm{mL} / \mathrm{g} \\
\text { (or other units or } \\
\% \text { sorbed) } \\
\end{array}$ & $\mathrm{CEC}(\mathrm{meq} / 100 \mathrm{~g})$ & $\begin{array}{l}\text { BET Area }\left(\mathrm{m}^{2} / \mathrm{g}\right) \\
\text { or Size (microns) }\end{array}$ & Solution Conditions & Notes & Reference \\
\hline $\begin{array}{l}\text { crystalline silicotitanate } \\
\text { (IONSIV IE-910, UOP) }\end{array}$ & trace Cs-137 & $910 \mathrm{~mL} / \mathrm{g}$ & & & $\begin{array}{l}\mathrm{pH}>14, \mathrm{t}=72 \mathrm{~h},[\mathrm{Na}]=5 \mathrm{M}, \\
{[\mathrm{K}]=0.5 \mathrm{M}, \mathrm{Na} / \mathrm{Cs}=8 \mathrm{e} 4} \\
\text { (Attachment } \mathrm{A} \text { for detailed } \\
\text { contents of DSSF waste } \\
\text { simulant) }\end{array}$ & $\begin{array}{l}12-14 \% \text { increase in Cs loading with } \\
25 \text {-fold dilution of waste (5M to } 0.2 \mathrm{M} \\
\mathrm{Na} \text { ) }\end{array}$ & Brown 1996 \\
\hline $\begin{array}{l}\text { zeolite (aluminosilicate, } \\
\text { IONSIV TIE-96, UOP) }\end{array}$ & trace Cs-137 & $21 \mathrm{~mL} / \mathrm{g}$ & & & $\begin{array}{l}\mathrm{pH}>14, \mathrm{t}=72 \mathrm{~h},[\mathrm{Na}]=5 \mathrm{M}, \\
{[\mathrm{K}]=0.5 \mathrm{M}, \mathrm{Na} / \mathrm{Cs}=8 \mathrm{e} 4} \\
\text { (Attachment A for detailed } \\
\text { contents of DSSF waste } \\
\text { simulant) }\end{array}$ & $\begin{array}{l}\text { double Cs loading with } 25 \text {-fold } \\
\text { dilution of waste ( } 5 \mathrm{M} \text { to } 0.2 \mathrm{M} \mathrm{Na} \text { ) }\end{array}$ & Brown 1996 \\
\hline $\begin{array}{l}\text { polymeric macrocycle } \\
\text { (SuperLig 644, IBC } \\
\text { Advanced Technol.) }\end{array}$ & trace Cs-137 & $500 \mathrm{~mL} / \mathrm{g}$ & & & $\begin{array}{l}\mathrm{pH}>14, \mathrm{t}=72 \mathrm{~h},[\mathrm{Na}]=5 \mathrm{M}, \\
{[\mathrm{K}]=0.5 \mathrm{M}, \mathrm{Na} / \mathrm{Cs}=8 \mathrm{e} 4} \\
\text { (Attachment A for detailed } \\
\text { contents of DSSF waste } \\
\text { simulant) }\end{array}$ & $\begin{array}{l}40-45 \% \text { decrease in Cs loading with } \\
25 \text {-fold dilution of waste ( } 5 \mathrm{M} \text { to } 0.2 \mathrm{M} \\
\mathrm{Na} \text { ) }\end{array}$ & Brown 1996 \\
\hline $\begin{array}{l}\text { resorcinol-formaldehyde } \\
\text { resin (BSC-187-210, } \\
\text { Boulder Sci.) }\end{array}$ & trace Cs-137 & $220 \mathrm{~mL} / \mathrm{g}$ & & & $\begin{array}{l}\mathrm{pH}>14, \mathrm{t}=72 \mathrm{~h},[\mathrm{Na}]=5 \mathrm{M}, \\
{[\mathrm{K}]=0.5 \mathrm{M}, \mathrm{Na} / \mathrm{Cs}=8 \mathrm{e} 4} \\
\text { (Attachment A for detailed } \\
\text { contents of DSSF waste } \\
\text { simulant) }\end{array}$ & $\begin{array}{l}40-45 \% \text { decrease in Cs loading with } \\
25 \text {-fold dilution of waste ( } 5 \mathrm{M} \text { to } 0.2 \mathrm{M} \\
\mathrm{Na} \text { ) }\end{array}$ & Brown 1996 \\
\hline $\begin{array}{l}\text { phenol-formaldehyde } \\
\text { resin (Duolite CS-100, } \\
\text { Rohm \& Hass) }\end{array}$ & trace Cs-137 & $88 \mathrm{~mL} / \mathrm{g}$ & & & $\begin{array}{l}\mathrm{pH}>14, \mathrm{t}=72 \mathrm{~h},[\mathrm{Na}]=5 \mathrm{M}, \\
{[\mathrm{K}]=0.5 \mathrm{M}, \mathrm{Na} / \mathrm{Cs}=8 \mathrm{e} 4} \\
\text { (Attachment A for detailed } \\
\text { contents of DSSF waste } \\
\text { simulant) }\end{array}$ & $\begin{array}{l}40-45 \% \text { decrease in Cs loading with } \\
25 \text {-fold dilution of waste ( } 5 \mathrm{M} \text { to } 0.2 \mathrm{M} \\
\mathrm{Na} \text { ) }\end{array}$ & Brown 1996 \\
\hline kaolinite & $1 \mathrm{kBq} / \mathrm{mL}$ & 3,287 & 170 & $<2 u m$ & $\begin{array}{l}{[\mathrm{CaCl} 2]=0.01 \mathrm{M}, \mathrm{pH} \sim 7,} \\
\mathrm{t}=24 \mathrm{~h}, 1 \mathrm{~g} \text { clay } / \mathrm{L}\end{array}$ & CEC in mol_c/kg & $\begin{array}{l}\text { Bellenger } \\
(2008)\end{array}$ \\
\hline montmorillonite & $1 \mathrm{kBq} / \mathrm{mL}$ & 4,514 & 940 & $<2 u m$ & $\begin{array}{l}{[\mathrm{CaCl} 2]=0.01 \mathrm{M}, \mathrm{pH} \sim 7} \\
\mathrm{t}=24 \mathrm{~h}, 1 \mathrm{~g} \text { clay } / \mathrm{L}\end{array}$ & CEC in mol_c/kg & $\begin{array}{l}\text { Bellenger } \\
(2008)\end{array}$ \\
\hline
\end{tabular}


TABLE 2.3 (Cont.)

\begin{tabular}{|c|c|c|c|c|c|c|c|}
\hline Sorbent Material & $\begin{array}{c}\text { Concentration } \\
\text { of Element }\end{array}$ & $\begin{array}{l}\mathrm{K}_{\mathrm{d}} \text { in } \mathrm{mL} / \mathrm{g} \\
\text { (or other units or } \\
\% \text { sorbed) }\end{array}$ & CEC (meq/100g) & $\begin{array}{l}\text { BET Area }\left(\mathrm{m}^{2} / \mathrm{g} \text { ) }\right. \\
\text { or Size (microns) }\end{array}$ & Solution Conditions & Notes & Reference \\
\hline illite & $5 \mathrm{kBq} / \mathrm{mL}$ & 155,000 & 300 & $<2 u m$ & $\begin{array}{l}{[\mathrm{CaCl} 2]=0.01 \mathrm{M}, \mathrm{pH} \sim 7} \\
\mathrm{t}=24 \mathrm{~h}, 1 \mathrm{~g} \text { clay } / \mathrm{L}\end{array}$ & CEC in mol_c/kg & $\begin{array}{l}\text { Bellenger } \\
(2008)\end{array}$ \\
\hline quartz & $1 \mathrm{kBq} / \mathrm{mL}$ & 0 & & $<200$ um & $\begin{array}{l}{[\mathrm{CaCl} 2]=0.01 \mathrm{M}, \mathrm{pH} \sim 7} \\
\mathrm{t}=24 \mathrm{~h}, 10 \mathrm{~g} / \mathrm{L}\end{array}$ & CEC in mol_c/kg & $\begin{array}{l}\text { Bellenger } \\
(2008)\end{array}$ \\
\hline calcium carbonate & $1 \mathrm{kBq} / \mathrm{mL}$ & 33.8 & & & $\begin{array}{l}{[\mathrm{CaCl} 2]=0.01 \mathrm{M}, \mathrm{pH} \sim 7} \\
\mathrm{t}=24 \mathrm{~h}, 10 \mathrm{~g} / \mathrm{L}\end{array}$ & CEC in mol_c/kg & $\begin{array}{l}\text { Bellenger } \\
(2008)\end{array}$ \\
\hline
\end{tabular}

\section{TABLE 2-3 Notes and citations:}

1. Ahmad and Qureshi (1989), "Separation of RNs from liquid waste using a mineral mixture," Sep. Sci. Technol., 24(7-8), 569-579.

2. Spalding and Jacobs, private communication, Oak Ridge National Laboratory, ES Division.

3. Bakunov and Yudintseva (1989), "Decreased accumulation of Cs-137 in plants following amendment of soils with natural sorbents," Agrokhimiya, 6, 90-96 [in Russian].

4. Manos and Kanatzidis (2009), J. Amer. Chem. Soc., 131, 6599-6607.

5. Rehspringer, Estournes, and Vilminot (2004), "Process for containment of cesium and/or rubidium in fritted ceramic matrices based on expanded perlite," FR 2844913 A1 20040326 [French patent].

6. Sabodina et al. (2006), J. Radioanal. Nucl. Chem., 270, 349-355.

7. Mishra and Vijaya (2007), "Removal behavior of hydrous manganese oxide and hydrous stannic oxide for Cs(I) ions from aqueous solutions," Sep. Purif. Technol. 54, 10-17.

8. Murali and Mathur (2002), "Sorption chars of Am, Sr, Cs on bentonite and granite," J. Radioanal. Nucl. Chem., 254(1), 129-136. Refer to Refs 18, 19 for Am complexing agents.

9. McKinley and Scholtis (1993), “A comparison of radionuclide sorption databases used inrecent performance assessments," J. Contam. Hydrol., 13, 347-363 (Table 3, data from Refs. 1-6).

10. McKinley and Scholtis (1993), “A comparison of radionuclide sorption databases used inrecent performance assessments," J. Contam. Hydrol., 13, 347-363 (Table 4, data from Ref. 9, Vieno and Suolanen, 1991).

11. Wahlberg and Fishman (1962), "Adsorption of Cs on clay minerals," USGS Bulletin, Vol 1140-A.

12. Wendling et al. (2004), "Cesium sorption to illite as affected by oxalate," Clays and Clay Minerals, 52(3), 375-381. 


\section{TABLE 2-3 Notes and citations (Cont.):}

13. Lujaniene et al. (2009), "Application of inorganic sorbents for removal of Cs Sr Pu and Am from contaminated solutions," J. Radioanl. Nucl. Chem. 282, 787-791.

14. Behrens et al. (1998), "Assessment of a sodium nonatitanate and pharmacosiderite-type ion exchangers for Sr and Cs removal from DOE waste simulants," Environ. Sci. Technol. 32, 101-107.

15. Marsh et al. (1995), "Effects of soluble organic complexants and their degradation products on the removal of selected radionuclides from high level waste, Part III," Los Alamos National Lab Report, LA-13000, 64 pgs.

16. Hassan et al. (2002), "Ion exchange removal of cesium from Hanford tank waste supernates with SuperLig 644 resin,” J. Radioanal. Nucl. Chem., 254, 33-40.

17. Marsh et al. (1995), "Effects of soluble organic complexants and their degradation products on the removal of selected radionuclides from high level waste, Part II," Los Alamos National Lab Report, LA-12943, 47 pgs.

18. Brown et al. (1996), "Comparison of Organic and inorganic ion exchangers for removal of Cs and Sr from simulated and actual Hanford 241-AW-101 DSSF tank waste," Pacific Northwest National Lab Report, PNNL-11120, 70 pgs.

19. Bellenger and Staunton (2008), "Adsorption and desorption of $85 \mathrm{Sr}$ and $137 \mathrm{Cs}$ on reference minerals with and without inorganic and organic surface coatings,” J. Environ. Radioactivity 99, 831-840. 
TABLE 2-4 Summary of the properties of sorbent candidates for americium in solution.

\begin{tabular}{|c|c|c|c|c|c|c|c|}
\hline Sorbent Material & $\begin{array}{c}\text { Concentration } \\
\text { of Element }\end{array}$ & $\begin{array}{l}\mathrm{K}_{\mathrm{d}} \text { in } \mathrm{mL} / \mathrm{g} \\
\text { (or other } \\
\text { units or } \\
\text { \% sorbed) }\end{array}$ & $\begin{array}{l}\text { CEC } \\
(\mathrm{meq} / \\
100 \mathrm{~g}) \\
\end{array}$ & $\begin{array}{c}\text { BET Area } \\
\left(\mathrm{m}^{2} / \mathrm{g}\right) \text { or Size } \\
(\text { microns }) \\
\end{array}$ & Solution Conditions & Notes & Reference \\
\hline $\begin{array}{l}\text { antimonysilicate } \\
\text { (INOES Silicas Ltd.) }\end{array}$ & trace & $\begin{array}{l}1000- \\
1,000,000\end{array}$ & & & $\begin{array}{l}{[\mathrm{H}+]=0.01-1 \mathrm{M}, \mathrm{t}=3 \mathrm{~d}} \\
\mathrm{~V} / \mathrm{M}=500 \mathrm{~mL} / \mathrm{g}\end{array}$ & $\begin{array}{l}\text { XRD pattern similar to hydrous antimony } \\
\text { pentoxide (antimonic acid, } \mathrm{Sb}_{2} \mathrm{O}_{5} 4 \mathrm{H}_{2} \mathrm{O} \text { ) a } \\
\text { typical pyrochlore structure }\end{array}$ & $\begin{array}{l}\text { Al-Attar } \\
(2003)\end{array}$ \\
\hline $\begin{array}{l}\text { antimonysilicate, } \\
\text { calcium form } \\
\text { (synthesized) }\end{array}$ & trace & 100,000 & & & $\begin{array}{l}{[\mathrm{Ca}+]=0.01 \mathrm{M}, \mathrm{t}=3 \mathrm{~d}} \\
\mathrm{~V} / \mathrm{M}=500 \mathrm{~mL} / \mathrm{g}\end{array}$ & $\begin{array}{l}\text { XRD patttern shows high crystallinity and } \\
\text { pyrochlore structure }\end{array}$ & $\begin{array}{l}\text { Al-Attar } \\
(2003)\end{array}$ \\
\hline $\begin{array}{l}\text { antimonysilicate, } \\
\text { sodium form } \\
\text { (synthesized) }\end{array}$ & trace & $1,000,000$ & & & $\begin{array}{l}{[\mathrm{H}+]=0.01-1 \mathrm{M}, \mathrm{t}=3 \mathrm{~d}} \\
\mathrm{~V} / \mathrm{M}=500 \mathrm{~mL} / \mathrm{g}\end{array}$ & $\begin{array}{l}\text { XRD patttern shows high crystallinity and } \\
\text { pyrochlore structure }\end{array}$ & $\begin{array}{l}\text { Al-Attar } \\
(2003)\end{array}$ \\
\hline $\begin{array}{l}\text { birnessite, acid form } \\
\text { (synthesized) }\end{array}$ & trace & $10-10,000$ & & & $\begin{array}{l}{[\mathrm{H}+]=0.01-1 \mathrm{M}, \mathrm{t}=3 \mathrm{~d}} \\
\mathrm{~V} / \mathrm{M}=500 \mathrm{~mL} / \mathrm{g}\end{array}$ & & $\begin{array}{l}\text { Al-Attar } \\
(2003)\end{array}$ \\
\hline $\begin{array}{l}\text { birnessite, calcium form } \\
\text { (synthesized; similar to } \\
\text { natural mineral) }\end{array}$ & trace & $6,000,000$ & & & $\begin{array}{l}{[\mathrm{Ca}+]=0.01 \mathrm{M}, \mathrm{t}=3 \mathrm{~d}} \\
\mathrm{~V} / \mathrm{M}=500 \mathrm{~mL} / \mathrm{g}\end{array}$ & $\begin{array}{l}\text { XRD pattern similar to natural Ca-rich } \\
\text { birnessite reported by Drits et al } 1997\end{array}$ & $\begin{array}{l}\text { Al-Attar } \\
(2003)\end{array}$ \\
\hline $\begin{array}{l}\text { birnessite, sodium form } \\
\left(\mathrm{Na}_{4} \mathrm{Mn}_{14} \mathrm{O}_{27} 9 \mathrm{H}_{2} \mathrm{O}\right. \\
\text { synthesized })\end{array}$ & trace & $\begin{array}{l}1,000,000- \\
30,000,000\end{array}$ & 310 & & $\begin{array}{l}{[\mathrm{Na}+]=0.01-1 \mathrm{M}, \mathrm{t}=3 \mathrm{~d}} \\
\mathrm{~V} / \mathrm{M}=1000 \mathrm{~mL} / \mathrm{g}\end{array}$ & & $\begin{array}{l}\text { Al-Attar } \\
(2003)\end{array}$ \\
\hline $\begin{array}{l}\text { titanosilicates } \\
\left(\mathrm{Na}_{2} \mathrm{Ti}_{2} \mathrm{O}_{3} \mathrm{SiO}_{4} 2 \mathrm{H}_{2} \mathrm{O},\right. \\
(\mathrm{Na}, \mathrm{K})_{2} \mathrm{TiSi}_{5} \mathrm{O}_{13} \mathrm{nH}_{2} \mathrm{O}, \\
\mathrm{Na}_{9} \mathrm{Si}_{12} \mathrm{O}_{38}(\mathrm{OH}) 12 \mathrm{H}_{2} \mathrm{O}, \\
\mathrm{Na}_{3}(\mathrm{Na}, \mathrm{H}) \mathrm{Ti}_{2} \mathrm{O}_{2}\left[\mathrm{Si}_{2} \mathrm{O}_{6}\right]_{2} \\
\left.2 \mathrm{H}_{2} \mathrm{O}\right) \text {, synthesized } \\
\text { materials })\end{array}$ & trace & $\begin{array}{l}10,000- \\
10,000,000\end{array}$ & $\begin{array}{l}310- \\
760\end{array}$ & 5-25 microns & $\begin{array}{l}{[\mathrm{Na}+]=0.01-1 \mathrm{M}, \mathrm{t}=3 \mathrm{~d}} \\
\mathrm{~V} / \mathrm{M}=1000 \mathrm{~mL} / \mathrm{g}, \mathrm{pH}=?\end{array}$ & $\begin{array}{l}\text { titanosilicates not tested in acidic media due } \\
\text { to low acid resistence, reported theoretical } \\
\text { CEC in meq/g }\end{array}$ & $\begin{array}{l}\text { Al-Attar } \\
(2003)\end{array}$ \\
\hline
\end{tabular}


TABLE 2-4 (Cont.)

\begin{tabular}{|c|c|c|c|c|c|c|c|}
\hline Sorbent Material & $\begin{array}{l}\text { Conc. of } \\
\text { Element }\end{array}$ & $\begin{array}{c}\mathrm{K}_{\mathrm{d}} \text { in } \mathrm{mL} / \mathrm{g} \\
\text { (or other } \\
\text { units or } \\
\% \text { sorbed) }\end{array}$ & $\begin{array}{l}\text { CEC } \\
\text { (meq/ } \\
100 \mathrm{~g}) \\
\end{array}$ & $\begin{array}{c}\text { BET Area } \\
\left(\mathrm{m}^{2} / \mathrm{g}\right) \text { or Size } \\
(\text { microns })\end{array}$ & Solution Conditions & Notes & Reference \\
\hline apatite & $2-5 e-9 M$ & 10,000 & & $0.04-0.06 \mathrm{~mm}$ & $\begin{array}{l}\mathrm{pH}=8, \mathrm{t}=5 \mathrm{~d},[\mathrm{HCO} 3- \\
]=123 \mathrm{mg} / \mathrm{L},[\mathrm{Na}]=65 \mathrm{mg} / \mathrm{L}, \\
\mathrm{V} / \mathrm{m}=40-100 \mathrm{~mL} / \mathrm{g}, \text { actual } \\
\text { grdwater solution } \\
\text { composition (Table II) }\end{array}$ & Fig $1 \mathrm{~b}$, no change in $\mathrm{Kd}$ as inc $\mathrm{pH}$ from $4-9$ & $\begin{array}{l}\text { Allard } \\
(1984)\end{array}$ \\
\hline $\begin{array}{l}\text { attapulgite (or } \\
\text { palygorskite, } \\
\text { magnesium aluminium } \\
\text { phyllosilicate, } \\
(\mathrm{Mg}, \mathrm{Al})_{2} \mathrm{Si}_{4} \mathrm{O}_{10}(\mathrm{OH}) \\
\left.4\left(\mathrm{H}_{2} \mathrm{O}\right)\right)\end{array}$ & $2-5 e-9 M$ & 10,000 & & $0.04-0.06 \mathrm{~mm}$ & $\begin{array}{l}\mathrm{pH}=8, \mathrm{t}=5 \mathrm{~d},[\mathrm{HCO} 3- \\
]=123 \mathrm{mg} / \mathrm{L},[\mathrm{Na}]=65 \mathrm{mg} / \mathrm{L}, \\
\mathrm{V} / \mathrm{m}=40-100 \mathrm{~mL} / \mathrm{g}, \text { actual } \\
\text { grdwater solution } \\
\text { composition (Table II) }\end{array}$ & $\begin{array}{l}\text { Fig } 1 \mathrm{a} \text {, slight inc in } \mathrm{Kd} \text { for as inc } \mathrm{pH} \text { from } 4 \\
\text { to } 6 \text {, then constant }\end{array}$ & $\begin{array}{l}\text { Allard } \\
(1984)\end{array}$ \\
\hline biotite & $2-5 e-9 M$ & $\begin{array}{l}1,000- \\
10,000\end{array}$ & & $0.04-0.06 \mathrm{~mm}$ & $\begin{array}{l}\mathrm{pH}=8, \mathrm{t}=5 \mathrm{~d},[\mathrm{HCO} 3- \\
]=123 \mathrm{mg} / \mathrm{L},[\mathrm{Na}]=65 \mathrm{mg} / \mathrm{L}, \\
\mathrm{V} / \mathrm{m}=40-100 \mathrm{~mL} / \mathrm{g}, \text { actual } \\
\text { grdwater solution } \\
\text { composition (Table II) }\end{array}$ & $\begin{array}{l}\text { Fig } 1 \mathrm{a} \text {, inc in } \mathrm{Kd} \text { as inc } \mathrm{pH} \text { from } 4 \text { to } 7 \text {, } \\
\text { then constant }\end{array}$ & $\begin{array}{l}\text { Allard } \\
(1984)\end{array}$ \\
\hline calcite & $2-5 e-9 M$ & $\begin{array}{l}5,000- \\
50,000\end{array}$ & & $0.04-0.06 \mathrm{~mm}$ & $\begin{array}{l}\mathrm{pH}=8, \mathrm{t}=5 \mathrm{~d},[\mathrm{HCO} 3- \\
]=123 \mathrm{mg} / \mathrm{L},[\mathrm{Na}]=65 \mathrm{mg} / \mathrm{L}, \\
\mathrm{V} / \mathrm{m}=40-100 \mathrm{~mL} / \mathrm{g}, \text { actual } \\
\text { grdwater solution } \\
\text { composition (Table II) }\end{array}$ & Fig $1 b, \mathrm{Kd}$ incs with $\mathrm{pH}$ from $7-9$ & $\begin{array}{l}\text { Allard } \\
(1984)\end{array}$ \\
\hline $\begin{array}{l}\text { corundum (aluminium } \\
\text { oxide, } \mathrm{Al}_{2} \mathrm{O}_{3} \text { ) }\end{array}$ & $2-5 e-9 M$ & $10-5,000$ & & $0.04-0.06 \mathrm{~mm}$ & $\begin{array}{l}\mathrm{pH}=8, \mathrm{t}=5 \mathrm{~d},[\mathrm{HCO} 3- \\
]=123 \mathrm{mg} / \mathrm{L},[\mathrm{Na}]=65 \mathrm{mg} / \mathrm{L}, \\
\mathrm{V} / \mathrm{m}=40-100 \mathrm{~mL} / \mathrm{g}, \text { actual } \\
\text { grdwater solution } \\
\text { composition (Table II) }\end{array}$ & $\begin{array}{l}\text { Fig 1b, Kd incs with pH from 4-7, Kd_max } \\
\sim \text { pH 7-9 }\end{array}$ & $\begin{array}{l}\text { Allard } \\
(1984)\end{array}$ \\
\hline
\end{tabular}


TABLE 2-4 (Cont.)

\begin{tabular}{|c|c|c|c|c|c|c|c|}
\hline Sorbent Material & $\begin{array}{l}\text { Conc. of } \\
\text { Element }\end{array}$ & $\begin{array}{l}\mathrm{K}_{\mathrm{d}} \text { in } \mathrm{mL} / \mathrm{g} \\
\text { (or other } \\
\text { units or } \\
\% \text { sorbed) }\end{array}$ & $\begin{array}{l}\text { CEC } \\
\text { (meq/ } \\
100 g) \\
\end{array}$ & $\begin{array}{c}\text { BET Area } \\
\left(\mathrm{m}^{2} / \mathrm{g}\right) \text { or Size } \\
(\text { microns })\end{array}$ & Solution Conditions & Notes & Reference \\
\hline fluorite & $2-5 e-9 M$ & 8,000 & & $0.04-0.06 \mathrm{~mm}$ & $\begin{array}{l}\mathrm{pH}=8, \mathrm{t}=5 \mathrm{~d},[\mathrm{HCO}- \\
]=123 \mathrm{mg} / \mathrm{L},[\mathrm{Na}]=65 \mathrm{mg} / \mathrm{L}, \\
\mathrm{V} / \mathrm{m}=40-100 \mathrm{~mL} / \mathrm{g}, \text { actual } \\
\text { grdwater solution } \\
\text { composition (Table II) }\end{array}$ & Fig $1 b$, no change in $\mathrm{Kd}$ as inc $\mathrm{pH}$ from $4-9$ & $\begin{array}{l}\text { Allard } \\
(1984)\end{array}$ \\
\hline $\begin{array}{l}\text { gibbsite (aluminium } \\
\left.\text { hydroxide, } \mathrm{Al}(\mathrm{OH})_{3}\right)\end{array}$ & $2-5 e-9 M$ & $60-60,000$ & & $0.04-0.06 \mathrm{~mm}$ & $\begin{array}{l}\mathrm{pH}=8, \mathrm{t}=5 \mathrm{~d},[\mathrm{HCO}- \\
]=123 \mathrm{mg} / \mathrm{L},[\mathrm{Na}]=65 \mathrm{mg} / \mathrm{L}, \\
\mathrm{V} / \mathrm{m}=40-100 \mathrm{~mL} / \mathrm{g}, \text { actual } \\
\text { grdwater solution } \\
\text { composition (Table II) }\end{array}$ & $\begin{array}{l}\text { Fig } 1 b, \text { Kd incs with } \mathrm{pH} \text { from 4-6, Kd_max } \\
\sim \mathrm{pH} \text { 6-9 }\end{array}$ & $\begin{array}{l}\text { Allard } \\
(1984)\end{array}$ \\
\hline $\begin{array}{l}\text { limonite (hydrous basic } \\
\text { iron oxide, } \mathrm{FeO}(\mathrm{OH}) \\
\mathrm{nH} 2 \mathrm{O})\end{array}$ & $2-5 e-9 M$ & $500-20,000$ & & $0.04-0.06 \mathrm{~mm}$ & $\begin{array}{l}\mathrm{pH}=8, \mathrm{t}=5 \mathrm{~d},[\mathrm{HCO}- \\
]=123 \mathrm{mg} / \mathrm{L},[\mathrm{Na}]=65 \mathrm{mg} / \mathrm{L}, \\
\mathrm{V} / \mathrm{m}=40-100 \mathrm{~mL} / \mathrm{g}, \text { actual } \\
\text { grdwater solution } \\
\text { composition (Table II) }\end{array}$ & $\begin{array}{l}\text { Fig } 1 \mathrm{~b}, \mathrm{Kd} \text { incs with } \mathrm{pH} \text { from } 4-9, \mathrm{Kd} \_ \text {max } \\
\sim \mathrm{pH} 7\end{array}$ & $\begin{array}{l}\text { Allard } \\
(1984)\end{array}$ \\
\hline $\begin{array}{l}\text { magnetite (iron oxide, } \\
\mathrm{Fe}_{3} \mathrm{O}_{4} \text { ) }\end{array}$ & $2-5 e-9 M$ & $10-20,000$ & & $0.04-0.06 \mathrm{~mm}$ & $\begin{array}{l}\mathrm{pH}=8, \mathrm{t}=5 \mathrm{~d},[\mathrm{HCO}- \\
]=123 \mathrm{mg} / \mathrm{L},[\mathrm{Na}]=65 \mathrm{mg} / \mathrm{L}, \\
\mathrm{V} / \mathrm{m}=40-100 \mathrm{~mL} / \mathrm{g}, \text { actual } \\
\text { grdwater solution } \\
\text { composition (Table II) }\end{array}$ & $\begin{array}{l}\text { Fig } 1 b, \text { Kd incs with } \mathrm{pH} \text { from } 4-7, \mathrm{Kd} \_\max \\
\sim \mathrm{pH} 7-9\end{array}$ & $\begin{array}{l}\text { Allard } \\
(1984)\end{array}$ \\
\hline $\begin{array}{l}\text { montmorillonite } \\
\left((\mathrm{Na}, \mathrm{Ca})_{0.3}(\mathrm{Al}, \mathrm{Mg})_{2} \mathrm{Si}_{4} \mathrm{O}\right. \\
\left.{ }_{10}(\mathrm{OH})_{2} \mathrm{nH}_{2} \mathrm{O}\right)\end{array}$ & $2-5 e-9 M$ & 10,000 & & $0.04-0.06 \mathrm{~mm}$ & $\begin{array}{l}\mathrm{pH}=4-9, \mathrm{t}=5 \mathrm{~d},[\mathrm{HCO}- \\
]=123 \mathrm{mg} / \mathrm{L},[\mathrm{Na}]=65 \mathrm{mg} / \mathrm{L}, \\
\mathrm{V} / \mathrm{m}=40-100 \mathrm{~mL} / \mathrm{g}, \text { actual } \\
\text { grdwater solution } \\
\text { composition }(\text { Table } \mathrm{II})\end{array}$ & Fig 1a, no dep of $\mathrm{Kd}$ for $\mathrm{pH}$ range of $4-9$ & $\begin{array}{l}\text { Allard } \\
(1984)\end{array}$ \\
\hline
\end{tabular}


TABLE 2-4 (Cont.)

\begin{tabular}{|c|c|c|c|c|c|c|c|}
\hline Sorbent Material & $\begin{array}{l}\text { Conc. of } \\
\text { Element }\end{array}$ & $\begin{array}{l}\mathrm{K}_{\mathrm{d}} \text { in } \mathrm{mL} / \mathrm{g} \\
\text { (or other } \\
\text { units or } \\
\% \text { sorbed) }\end{array}$ & $\begin{array}{l}\text { CEC } \\
(\mathrm{meq} / \\
100 \mathrm{~g})\end{array}$ & $\begin{array}{c}\text { BET Area } \\
\left(\mathrm{m}^{2} / \mathrm{g}\right) \text { or Size } \\
(\text { microns })\end{array}$ & Solution Conditions & Notes & Reference \\
\hline
\end{tabular}

clay (Suez Gulf, Egypt,

predominantly quartz-

$48 \%$, kaolinite- $11 \%$ ?,

$40 \% \mathrm{Ca}-$

montmorillonite)

olivine (magnesium iron $\quad 2-5 \mathrm{e}-9 \mathrm{M} \quad 100-10,000$

silicate, $\left.(\mathrm{Mg}, \mathrm{Fe})_{2} \mathrm{SiO}_{4}\right)$

$\mathrm{FeS}_{2}$ )

2-5e-9M

$100-10,000$

2.35

(microns)

Solution Conditions

CEC based upon MB dye adsorption where

$\mathrm{MB}=320 \mathrm{~g} / \mathrm{mol}$ for dehydrated dye

(C16N18N3SCl)

200 mesh $\quad \mathrm{V} / \mathrm{m}=100 \mathrm{~mL} / \mathrm{g}$

$\mathrm{mol} / \mathrm{g}$

V/m=100

$0.04-0.06 \mathrm{~mm}$

$\mathrm{pH}=8, \mathrm{t}=5 \mathrm{~d}$, [HCO3-

]$=123 \mathrm{mg} / \mathrm{L},[\mathrm{Na}]=65 \mathrm{mg} / \mathrm{L}$,

Fig 1a, inc in $\mathrm{Kd}$ as inc $\mathrm{pH}$ from 5 to 9

$\mathrm{V} / \mathrm{m}=40-100 \mathrm{~mL} / \mathrm{g}$, actual

grdwater solution

composition (Table II)

$0.04-0.06 \mathrm{~mm}$

$\mathrm{pH}=8, \mathrm{t}=5 \mathrm{~d}$, [HCO3-

]$=123 \mathrm{mg} / \mathrm{L},[\mathrm{Na}]=65 \mathrm{mg} / \mathrm{L}$

Allard

$\mathrm{V} / \mathrm{m}=40-100 \mathrm{~mL} / \mathrm{g}$, actual

grdwater solution

composition (Table II)

quartz

$2-5 e-9 M$

$50-1,000$

$0.04-0.06 \mathrm{~mm}$

$\mathrm{pH}=8, \mathrm{t}=5 \mathrm{~d}$, [HCO3-

]$=123 \mathrm{mg} / \mathrm{L},[\mathrm{Na}]=65 \mathrm{mg} / \mathrm{L}$,

$\mathrm{V} / \mathrm{m}=40-100 \mathrm{~mL} / \mathrm{g}$, actual

grdwater solution

composition (Table II)

2-5e-9M

0.04-0.06mm

$\mathrm{pH}=8, \mathrm{t}=5 \mathrm{~d},[\mathrm{HCO} 3$

]$=123 \mathrm{mg} / \mathrm{L},[\mathrm{Na}]=65 \mathrm{mg} / \mathrm{L}$,

$\mathrm{V} / \mathrm{m}=40-100 \mathrm{~mL} / \mathrm{g}$, actual

grdwater solution

chemistry (hydrolysis) rather than sorbent

properties (influence of $\mathrm{pH}$ on sorption did not vary for range of minerals) 
TABLE 2-4 (Cont.)

\begin{tabular}{|c|c|c|c|c|c|c|c|}
\hline Sorbent Material & $\begin{array}{l}\text { Conc. of } \\
\text { Element } \\
\end{array}$ & $\begin{array}{c}\mathrm{K}_{\mathrm{d}} \text { in } \mathrm{mL} / \mathrm{g} \\
\text { (or other } \\
\text { units or } \\
\% \text { sorbed) } \\
\end{array}$ & $\begin{array}{l}\text { CEC } \\
\text { (meq/ } \\
100 \mathrm{~g}) \\
\end{array}$ & $\begin{array}{c}\text { BET Area } \\
\left(\mathrm{m}^{2} / \mathrm{g}\right) \text { or Size } \\
(\text { microns })\end{array}$ & Solution Conditions & Notes & Reference \\
\hline hematite & & 100,000 & & $\begin{array}{l}6.1 \mathrm{pCi} / \mathrm{mg} / \mathrm{min} \\
\text { in natural } \\
\text { groundwater; } \\
3.9 \mathrm{pCi} / \mathrm{mg} / \mathrm{min} \\
\text { in simulated } \\
\text { water }\end{array}$ & $\begin{array}{l}\text { natural and simulated } \\
\text { groundwater }\end{array}$ & & $\begin{array}{l}\text { Conca } \\
(2000)\end{array}$ \\
\hline montmorillonite & & 10,000 & & $\begin{array}{l}3.8 \mathrm{pCi} / \mathrm{mg} / \mathrm{min} \\
\text { in natural } \\
\text { groundwater; } \\
3.2 \mathrm{pCi} / \mathrm{mg} / \mathrm{min} \\
\text { in simulated } \\
\text { water }\end{array}$ & $\begin{array}{l}\text { natural and simulated } \\
\text { groundwater }\end{array}$ & & $\begin{array}{l}\text { Conca } \\
(2000)\end{array}$ \\
\hline $\begin{array}{l}\text { titanium silicate } \\
\text { (synthesized using } \\
\text { TiOSO }_{4} \text { ) }\end{array}$ & $3.2 \mathrm{e}-11 \mathrm{M}$ & $50-2,000$ & & $\begin{array}{l}120 \text { to } 726 \\
\mathrm{~m} 2 / \mathrm{g}\end{array}$ & $\begin{array}{l}\mathrm{I}=0.1 \mathrm{M} \mathrm{Na}+, 1 / 100 \text { to } \\
1 / 1000 \mathrm{~g} / \mathrm{mL}\end{array}$ & & $\begin{array}{l}\text { Lujaniene } \\
\text { (2009) }\end{array}$ \\
\hline geothite & $3 e-11 \mathrm{M}$ & $(45-95 \%)$ & & & $\begin{array}{l}\mathrm{pH}=3.5-8,[\text { sorbent }]=? \\
{[\mathrm{Na}]=0.1 \mathrm{M}}\end{array}$ & & $\begin{array}{l}\text { Lujaniene } \\
(2010)\end{array}$ \\
\hline hematite & $3 e-11 \mathrm{M}$ & $(35-95 \%)$ & & & $\begin{array}{l}\mathrm{pH}=3.5-8,[\text { sorbent }]=? \\
{[\mathrm{Na}]=0.1 \mathrm{M}}\end{array}$ & & $\begin{array}{l}\text { Lujaniene } \\
(2010)\end{array}$ \\
\hline magnetite & $3 e-11 \mathrm{M}$ & $(75-95 \%)$ & & & $\begin{array}{l}\mathrm{pH}=4-8,[\text { sorbent }]=?, \\
{[\mathrm{Na}]=0.1 \mathrm{M}}\end{array}$ & & $\begin{array}{l}\text { Lujaniene } \\
\text { (2010) }\end{array}$ \\
\hline $\begin{array}{l}\text { Triassic clay (Northern } \\
\text { Lithuania) }\end{array}$ & $3 e-11 \mathrm{M}$ & $(75-100 \%)$ & & & $\begin{array}{l}\mathrm{pH}=3.5-8,[\text { clay }]=64.3 \\
\mathrm{~m} 2 / \mathrm{L},[\mathrm{Na}]=0.01-0.1 \mathrm{M}\end{array}$ & $\begin{array}{l}\text { clay composition: } 56-71 \% \text { smectite }(14 \% \\
\text { montmorillonite, } 20 \% \text { illite, } 1-9 \% \text { chlorite } \\
\text { minerals) cemented by limonite } \\
(\mathrm{FeO}(\mathrm{OH}) \mathrm{nH} 2 \mathrm{O} \text {, surface coated with } 1.6 \% \\
\text { siderite }(\mathrm{FeCO}), 2.3 \% \text { hematie (a-Fe2O3), } \\
0.1 \% \text { goethite }(\mathrm{a}-\mathrm{FeOOH}), 19.8 \% \text { calcite }\end{array}$ & $\begin{array}{l}\text { Lujaniene } \\
(2010)\end{array}$ \\
\hline
\end{tabular}


TABLE 2-4 (Cont.)

\begin{tabular}{|c|c|c|c|c|c|c|c|}
\hline Sorbent Material & $\begin{array}{l}\text { Conc. of } \\
\text { Element } \\
\end{array}$ & $\begin{array}{c}\mathrm{K}_{\mathrm{d}} \text { in } \mathrm{mL} / \mathrm{g} \\
\text { (or other } \\
\text { units or } \\
\% \text { sorbed) }\end{array}$ & $\begin{array}{l}\text { CEC } \\
\text { (meq/ } \\
100 \mathrm{~g}) \\
\end{array}$ & $\begin{array}{c}\text { BET Area } \\
\left(\mathrm{m}^{2} / \mathrm{g}\right) \text { or Size } \\
(\text { microns })\end{array}$ & Solution Conditions & Notes & Reference \\
\hline bone char & $10^{-8}-10^{-7} \mathrm{M}$ & $9-800$ & & & $\begin{array}{l}\mathrm{pH}=14, \mathrm{t}=0.5,2,6 \mathrm{~h}, \\
{[\mathrm{Na}]=4 \mathrm{M},[\mathrm{Al}]=0.4 \mathrm{M},} \\
{[\mathrm{CO} 32-]=2 \mathrm{M},[\mathrm{TOC}]=0.1-} \\
0.7 \mathrm{M}, \text { four Hanford Tank } \\
\text { 101-SY simulant solutions } \\
\text { (Table } 1 \text { for additional } \\
\text { minor components) }\end{array}$ & $\begin{array}{l}\text { Table } 8 \text { summarizes time dependency of } \\
\text { Kd's with various solutions; significantly } \\
\text { lower Kd's in presence of organic } \\
\text { complexants (untreated solutions); } \\
\text { complexation of Am in highly alkaline } \\
\text { solns with simpler organic cmpds (such as } \\
\text { oxalate and acetate formed from radiolysis } \\
\text { of EDTA); converted [Am] to molarity }\end{array}$ & $\begin{array}{l}\text { Marsh } \\
(1995)\end{array}$ \\
\hline $\begin{array}{l}\text { bone char (Stauffer } \\
\text { Chem. Co.) }\end{array}$ & $10^{-7} \mathrm{M}$ & $0.3-5,000$ & & & $\begin{array}{l}\mathrm{pH}=12-13, \mathrm{t}=0.5,2,6 \mathrm{~h}, \\
{[\mathrm{Na}]=2 \mathrm{M},[\mathrm{Al}]=0.01-0.2,} \\
{[\mathrm{CO} 32-]=0.2-0.6 \mathrm{M},} \\
{[\mathrm{TOC}]=0.1-1 \mathrm{M}, \text { four } 3: 1} \\
\text { diluted Hanford complexant } \\
\text { conc simulant solutions } \\
\text { (Table } 1 \text { for additional } \\
\text { minor components) }\end{array}$ & $\begin{array}{l}\text { Table } 9 \text { summarizes time dependency of } \\
\text { Kd's with various solutions; significantly } \\
\text { lower Kd's in presence of organic } \\
\text { complexants (untreated solutions); } \\
\text { complexation of Am in highly alkaline } \\
\text { solns with simpler organic cmpds (such as } \\
\text { oxalate and acetate formed from radiolysis } \\
\text { of EDTA); converted [Am] to molarity }\end{array}$ & $\begin{array}{l}\text { Marsh } \\
\text { (1995) }\end{array}$ \\
\hline $\begin{array}{l}\text { clinoptilolite (natural } \\
\text { zeolite containing } \\
\text { quartz, feldspar and clay } \\
\text { from Castle Creek and } \\
\text { purified form) }\end{array}$ & $10^{-8}-10^{-7} \mathrm{M}$ & $2-600$ & & $<200$ mesh & $\begin{array}{l}\mathrm{pH}=14, \mathrm{t}=0.5,2,6 \mathrm{~h}, \\
{[\mathrm{Na}]=4 \mathrm{M},[\mathrm{Al}]=0.4 \mathrm{M},} \\
{[\mathrm{CO} 32-]=2 \mathrm{M},[\mathrm{TOC}]=0.1-} \\
0.7 \mathrm{M}, \text { four Hanford Tank } \\
\text { 101-SY simulant solutions } \\
\text { (Table } 1 \text { for additional } \\
\text { minor components) }\end{array}$ & $\begin{array}{l}\text { Table } 8 \text { summarizes time dependency of } \\
\text { Kd's with various solutions; significantly } \\
\text { lower Kd's in presence of organic } \\
\text { complexants (untreated solutions); } \\
\text { complexation of Am in highly alkaline } \\
\text { solns with simpler organic cmpds (such as } \\
\text { oxalate and acetate formed from radiolysis } \\
\text { of EDTA); converted [Am] to molarity }\end{array}$ & $\begin{array}{l}\text { Marsh } \\
(1995)\end{array}$ \\
\hline
\end{tabular}


TABLE 2-4 (Cont.)

\begin{tabular}{|c|c|c|c|c|c|c|c|}
\hline Sorbent Material & $\begin{array}{l}\text { Conc. of } \\
\text { Element }\end{array}$ & $\begin{array}{l}\mathrm{K}_{\mathrm{d}} \text { in } \mathrm{mL} / \mathrm{g} \\
\text { (or other } \\
\text { units or } \\
\% \text { sorbed) } \\
\end{array}$ & $\begin{array}{l}\text { CEC } \\
\text { (meq/ } \\
100 \mathrm{~g}) \\
\end{array}$ & $\begin{array}{c}\text { BET Area } \\
\left(\mathrm{m}^{2} / \mathrm{g}\right) \text { or Size } \\
(\text { microns }) \\
\end{array}$ & Solution Conditions & Notes & Reference \\
\hline $\begin{array}{l}\text { crystalline antimonic } \\
\text { acid composite (CsbA- } \\
\text { PAN, prepd by F. } \\
\text { Sebesta, Czech Techn. } \\
\text { Univ.) }\end{array}$ & $10^{-7} \mathrm{M}$ & $0.4-5,000$ & & & $\begin{array}{l}\mathrm{pH}=12-13, \mathrm{t}=0.5,2,6 \mathrm{~h}, \\
{[\mathrm{Na}]=2 \mathrm{M},[\mathrm{Al}]=0.01-0.2,} \\
{[\mathrm{CO} 32-]=0.2-0.6 \mathrm{M},} \\
{[\mathrm{TOC}]=0.1-1 \mathrm{M}, \text { four } 3: 1} \\
\text { diluted Hanford complexant } \\
\text { conc simulant solutions } \\
\text { (Table } 1 \text { for additional } \\
\text { minor components) }\end{array}$ & $\begin{array}{l}\text { Table } 9 \text { summarizes time dependency of } \\
\text { Kd's with various solutions; significantly } \\
\text { lower Kd's in presence of organic } \\
\text { complexants (untreated solutions); } \\
\text { complexation of Am in highly alkaline } \\
\text { solns with simpler organic cmpds (such as } \\
\text { oxalate and acetate formed from radiolysis } \\
\text { of EDTA); converted [Am] to molarity }\end{array}$ & $\begin{array}{l}\text { Marsh } \\
(1995)\end{array}$ \\
\hline $\begin{array}{l}\text { diphonix resin (Eichrom } \\
\text { Industries) }\end{array}$ & $10^{-7} \mathrm{M}$ & $0.4-4,000$ & & & $\begin{array}{l}\mathrm{pH}=12-13, \mathrm{t}=0.5,2,6 \mathrm{~h}, \\
{[\mathrm{Na}]=2 \mathrm{M},[\mathrm{Al}]=0.01-0.2,} \\
{[\mathrm{CO} 32-]=0.2-0.6 \mathrm{M},} \\
{[\mathrm{TOC}]=0.1-1 \mathrm{M}, \text { four } 3: 1} \\
\text { diluted Hanford complexant } \\
\text { conc simulant solutions } \\
\text { (Table } 1 \text { for additional } \\
\text { minor components) }\end{array}$ & $\begin{array}{l}\text { Table } 9 \text { summarizes time dependency of } \\
\text { Kd's with various solutions; significantly } \\
\text { lower Kd's in presence of organic } \\
\text { complexants (untreated solutions); } \\
\text { complexation of Am in highly alkaline } \\
\text { solns with simpler organic cmpds (such as } \\
\text { oxalate and acetate formed from radiolysis } \\
\text { of EDTA); converted [Am] to molarity }\end{array}$ & $\begin{array}{l}\text { Marsh } \\
\text { (1995) }\end{array}$ \\
\hline $\begin{array}{l}\text { hydrous titanium } \\
\text { dioxide (SNL/HTO, } \\
\text { amorphous form, prepd } \\
\text { by Sandia) }\end{array}$ & $10^{-8}-10^{-7} \mathrm{M}$ & $20-800$ & & fine powder & $\begin{array}{l}\mathrm{pH}=14, \mathrm{t}=0.5,2,6 \mathrm{~h}, \\
{[\mathrm{Na}]=4 \mathrm{M},[\mathrm{Al}]=0.4 \mathrm{M},} \\
{[\mathrm{CO} 32-]=2 \mathrm{M},[\mathrm{TOC}]=0.1-} \\
0.7 \mathrm{M}, \text { four Hanford Tank } \\
\text { 101-SY simulant solutions } \\
\text { (Table } 1 \text { for additional } \\
\text { minor components) }\end{array}$ & $\begin{array}{l}\text { Table } 8 \text { summarizes time dependency of } \\
\text { Kd's with various solutions; significantly } \\
\text { lower Kd's in presence of organic } \\
\text { complexants (untreated solutions); } \\
\text { complexation of Am in highly alkaline } \\
\text { solns with simpler organic cmpds (such as } \\
\text { oxalate and acetate formed from radiolysis } \\
\text { of EDTA); converted [Am] to molarity }\end{array}$ & $\begin{array}{l}\text { Marsh } \\
(1995)\end{array}$ \\
\hline
\end{tabular}


TABLE 2-4 (Cont.)

\begin{tabular}{|c|c|c|c|c|c|c|c|}
\hline Sorbent Material & $\begin{array}{l}\text { Conc. of } \\
\text { Element }\end{array}$ & $\begin{array}{l}\mathrm{K}_{\mathrm{d}} \text { in } \mathrm{mL} / \mathrm{g} \\
\text { (or other } \\
\text { units or } \\
\% \text { sorbed) }\end{array}$ & $\begin{array}{l}\text { CEC } \\
\text { (meq/ } \\
100 \mathrm{~g}) \\
\end{array}$ & $\begin{array}{c}\text { BET Area } \\
\left(\mathrm{m}^{2} / \mathrm{g}\right) \text { or Size } \\
(\text { microns }) \\
\end{array}$ & Solution Conditions & Notes & Reference \\
\hline $\begin{array}{l}\text { Ionsiv IE-96 (zeolite, } \\
\text { UOP) }\end{array}$ & $10^{-8}-10^{-7} \mathrm{M}$ & $0.3-400$ & & & $\begin{array}{l}\mathrm{pH}=14, \mathrm{t}=0.5,2,6 \mathrm{~h}, \\
{[\mathrm{Na}]=4 \mathrm{M},[\mathrm{Al}]=0.4 \mathrm{M},} \\
{[\mathrm{CO} 32-]=2 \mathrm{M},[\mathrm{TOC}]=0.1-} \\
0.7 \mathrm{M}, \text { four Hanford Tank } \\
\text { 101-SY simulant solutions } \\
\text { (Table } 1 \text { for additional } \\
\text { minor components) }\end{array}$ & $\begin{array}{l}\text { Table } 8 \text { summarizes time dependency of } \\
\text { Kd's with various solutions; significantly } \\
\text { lower Kd's in presence of organic } \\
\text { complexants (untreated solutions); } \\
\text { complexation of Am in highly alkaline } \\
\text { solns with simpler organic cmpds (such as } \\
\text { oxalate and acetate formed from radiolysis } \\
\text { of EDTA); converted [Am] to molarity }\end{array}$ & $\begin{array}{l}\text { Marsh } \\
(1995)\end{array}$ \\
\hline $\begin{array}{l}\text { monosodium titanate } \\
\text { (MST slurry, BSC-265- } \\
\text { 0106, Boulder Sci. Co.) }\end{array}$ & $10^{-7} \mathrm{M}$ & $0.2-8,000$ & & & $\begin{array}{l}\mathrm{pH}=12-13, \mathrm{t}=0.5,2,6 \mathrm{~h}, \\
{[\mathrm{Na}]=2 \mathrm{M},[\mathrm{Al}]=0.01-0.2,} \\
{[\mathrm{CO} 32-]=0.2-0.6 \mathrm{M},} \\
{[\mathrm{TOC}]=0.1-1 \mathrm{M}, \text { four } 3: 1} \\
\text { diluted Hanford complexant } \\
\text { conc simulant solutions } \\
\text { (Table } 1 \text { for additional } \\
\text { minor components) }\end{array}$ & $\begin{array}{l}\text { Table } 9 \text { summarizes time dependency of } \\
\text { Kd's with various solutions; significantly } \\
\text { lower Kd's in presence of organic } \\
\text { complexants (untreated solutions); } \\
\text { complexation of Am in highly alkaline } \\
\text { solns with simpler organic cmpds (such as } \\
\text { oxalate and acetate formed from radiolysis } \\
\text { of EDTA); converted [Am] to molarity }\end{array}$ & $\begin{array}{l}\text { Marsh } \\
(1995)\end{array}$ \\
\hline $\begin{array}{l}\text { Na nanotitanate (8104- } \\
170 \text { and } 8225-127, \\
\text { Allied Signal, Inc.) }\end{array}$ & $10^{-8}-10^{-7} \mathrm{M}$ & $6-800$ & & $\begin{array}{l}<100 \text { mesh } \\
(8104-170) \text { and } \\
<40 \text { mesh } \\
(8225-127)\end{array}$ & $\begin{array}{l}\mathrm{pH}=14, \mathrm{t}=0.5,2,6 \mathrm{~h}, \\
{[\mathrm{Na}]=4 \mathrm{M},[\mathrm{Al}]=0.4 \mathrm{M},} \\
{[\mathrm{CO} 32-]=2 \mathrm{M},[\mathrm{TOC}]=0.1-} \\
0.7 \mathrm{M}, \text { four Hanford Tank } \\
\text { 101-SY simulant solutions } \\
\text { (Table } 1 \text { for additional } \\
\text { minor components) }\end{array}$ & $\begin{array}{l}\text { Table } 8 \text { summarizes time dependency of } \\
\text { Kd's with various solutions; significantly } \\
\text { lower Kds in presence of organic } \\
\text { complexants (untreated solutions); } \\
\text { complexation of Am in highly alkaline } \\
\text { solns with simpler organic cmpds (such as } \\
\text { oxalate and acetate formed from radiolysis } \\
\text { of EDTA); converted [Am] to molarity }\end{array}$ & $\begin{array}{l}\text { Marsh } \\
(1995)\end{array}$ \\
\hline
\end{tabular}


TABLE 2-4 (Cont.)

\begin{tabular}{|c|c|c|c|c|c|c|c|}
\hline Sorbent Material & $\begin{array}{l}\text { Conc. of } \\
\text { Element }\end{array}$ & $\begin{array}{l}\mathrm{K}_{\mathrm{d}} \text { in } \mathrm{mL} / \mathrm{g} \\
\text { (or other } \\
\text { units or } \\
\% \text { sorbed) }\end{array}$ & $\begin{array}{l}\text { CEC } \\
\text { (meq/ } \\
100 \mathrm{~g}) \\
\end{array}$ & $\begin{array}{c}\text { BET Area } \\
\left(\mathrm{m}^{2} / \mathrm{g}\right) \text { or Size } \\
(\text { microns })\end{array}$ & Solution Conditions & Notes & Reference \\
\hline $\begin{array}{l}\text { nickel hexacyanoferrate } \\
\text { composite (NiFC-PAN, } \\
\text { prepd by F. Sebesta, } \\
\text { Czech Techn. Univ.) }\end{array}$ & $10^{-8}-10^{-7} \mathrm{M}$ & $40-1000$ & & & $\begin{array}{l}\mathrm{pH}=14, \mathrm{t}=0.5,2,6 \mathrm{~h}, \\
{[\mathrm{Na}]=4 \mathrm{M},[\mathrm{Al}]=0.4 \mathrm{M},} \\
{[\mathrm{CO} 32-]=2 \mathrm{M},[\mathrm{TOC}]=0.1-} \\
0.7 \mathrm{M}, \text { four Hanford Tank } \\
\text { 101-SY simulant solutions } \\
\text { (Table } 1 \text { for additional } \\
\text { minor components) }\end{array}$ & $\begin{array}{l}\text { Table } 8 \text { summarizes time dependency of } \\
\text { Kd's with various solutions; significantly } \\
\text { lower Kd's in presence of organic } \\
\text { complexants (untreated solutions); } \\
\text { complexation of Am in highly alkaline } \\
\text { solns with simpler organic cmpds (such as } \\
\text { oxalate and acetate formed from radiolysis } \\
\text { of EDTA); converted [Am] to molarity }\end{array}$ & $\begin{array}{l}\text { Marsh } \\
(1995)\end{array}$ \\
\hline $\begin{array}{l}\text { potassium } \\
\text { hexacyanoferrate } \\
(\mathrm{KCoFC})\end{array}$ & $10^{-8}-10^{-7} \mathrm{M}$ & $20-600$ & & & $\begin{array}{l}\mathrm{pH}=14, \mathrm{t}=0.5,2,6 \mathrm{~h}, \\
{[\mathrm{Na}]=4 \mathrm{M},[\mathrm{Al}]=0.4 \mathrm{M},} \\
{[\mathrm{CO} 32-]=2 \mathrm{M},[\mathrm{TOC}]=0.1-} \\
0.7 \mathrm{M}, \text { four Hanford Tank } \\
\text { 101-SY simulant solutions } \\
\text { (Table } 1 \text { for additional } \\
\text { minor components) }\end{array}$ & $\begin{array}{l}\text { Table } 8 \text { summarizes time dependency of } \\
\text { Kd's with various solutions; significantly } \\
\text { lower Kd's in presence of organic } \\
\text { complexants (untreated solutions); } \\
\text { complexation of Am in highly alkaline } \\
\text { solns with simpler organic cmpds (such as } \\
\text { oxalate and acetate formed from radiolysis } \\
\text { of EDTA); converted [Am] to molarity }\end{array}$ & $\begin{array}{l}\text { Marsh } \\
\text { (1995) }\end{array}$ \\
\hline $\begin{array}{l}\text { sodium nanotitanate } \\
\text { ( } 8104-170 \text { and } 8225- \\
127 \text {, Allied Signal, Inc.) }\end{array}$ & $10^{-7} \mathrm{M}$ & $0.3-8,000$ & & $\begin{array}{l}<100 \text { mesh } \\
(8104-170) \text { and } \\
<40 \text { mesh } \\
(8225-127)\end{array}$ & $\begin{array}{l}\mathrm{pH}=12-13, \mathrm{t}=0.5,2,6 \mathrm{~h}, \\
{[\mathrm{Na}]=2 \mathrm{M},[\mathrm{Al}]=0.01-0.2,} \\
{[\mathrm{CO} 32-]=0.2-0.6 \mathrm{M},} \\
{[\mathrm{TOC}]=0.1-1 \mathrm{M}, \text { four } 3: 1} \\
\text { diluted Hanford complexant } \\
\text { conc simulant solutions } \\
\text { (Table } 1 \text { for additional } \\
\text { minor components) }\end{array}$ & $\begin{array}{l}\text { Table } 9 \text { summarizes time dependency of } \\
\text { Kd's with various solutions; significantly } \\
\text { lower Kd's in presence of organic } \\
\text { complexants (untreated solutions); } \\
\text { complexation of Am in highly alkaline } \\
\text { solns with simpler organic cmpds (such as } \\
\text { oxalate and acetate formed from radiolysis } \\
\text { of EDTA); converted [Am] to molarity }\end{array}$ & $\begin{array}{l}\text { Marsh } \\
(1995)\end{array}$ \\
\hline
\end{tabular}


TABLE 2-4 (Cont.)

\begin{tabular}{|c|c|c|c|c|c|c|c|}
\hline Sorbent Material & $\begin{array}{l}\text { Conc. of } \\
\text { Element } \\
\end{array}$ & $\begin{array}{l}\mathrm{K}_{\mathrm{d}} \text { in } \mathrm{mL} / \mathrm{g} \\
\text { (or other } \\
\text { units or } \\
\% \text { sorbed) }\end{array}$ & $\begin{array}{l}\text { CEC } \\
\text { (meq/ } \\
100 \mathrm{~g}) \\
\end{array}$ & $\begin{array}{c}\text { BET Area } \\
\left(\mathrm{m}^{2} / \mathrm{g}\right) \text { or Size } \\
(\text { microns })\end{array}$ & Solution Conditions & Notes & Reference \\
\hline $\begin{array}{l}\text { titanium dioxide, } \\
\text { hydrous (SNL/HTO, } \\
\text { amorphous form, prepd } \\
\text { by Sandia) }\end{array}$ & $10^{-7} \mathrm{M}$ & $20-8,000$ & & fine powder & $\begin{array}{l}\mathrm{pH}=12-13, \mathrm{t}=0.5,2,6 \mathrm{~h}, \\
{[\mathrm{Na}]=2 \mathrm{M},[\mathrm{Al}]=0.01-0.2,} \\
{[\mathrm{CO} 32-]=0.2-0.6 \mathrm{M},} \\
{[\mathrm{TOC}]=0.1-1 \mathrm{M}, \text { four } 3: 1} \\
\text { diluted Hanford complexant } \\
\text { conc simulant solutions } \\
\text { (Table } 1 \text { for additional } \\
\text { minor components) }\end{array}$ & $\begin{array}{l}\text { Table } 9 \text { summarizes time dependency of } \\
\text { Kd's with various solutions; significantly } \\
\text { lower Kd's in presence of organic } \\
\text { complexants (untreated solutions); } \\
\text { complexation of Am in highly alkaline } \\
\text { solns with simpler organic cmpds (such as } \\
\text { oxalate and acetate formed from radiolysis } \\
\text { of EDTA); converted [Am] to molarity }\end{array}$ & $\begin{array}{l}\text { Marsh } \\
(1995)\end{array}$ \\
\hline bentonite & & 5,000 & & & & ref therein: McKinley and Hadermann 1985 & $\begin{array}{l}\text { McKinley } \\
(1993)\end{array}$ \\
\hline bentonite & & 29,400 & & & & ref therein: KBS 1983 & $\begin{array}{l}\text { McKinley } \\
(1993)\end{array}$ \\
\hline bentonite/sand & & 300 & & & & & $\begin{array}{l}\text { McKinley } \\
(1993)\end{array}$ \\
\hline cements & & $500-5000$ & & & & $\begin{array}{l}\text { converted } \mathrm{Kd} \text { to } \mathrm{mL} / \mathrm{g} \text {, reported as } 0.5 \text { to } \\
5 \mathrm{~m} 3 / \mathrm{kg}\end{array}$ & $\begin{array}{l}\text { McKinley } \\
(1993)\end{array}$ \\
\hline coastal marine water & & $1,000,000$ & & & & ref therein: Ashton and Summerling 1988 & $\begin{array}{l}\text { McKinley } \\
\text { (1993) }\end{array}$ \\
\hline pelagic clay & & $2,000,000$ & & & & ref therein: IAEA 1985 & $\begin{array}{l}\text { McKinley } \\
(1993)\end{array}$ \\
\hline sediment & & $2,000,000$ & & & & ref therein: Bushman et al. 1991 & $\begin{array}{l}\text { McKinley } \\
(1993)\end{array}$ \\
\hline soil/surface sediments & & 100,000 & & & & $\begin{array}{l}\text { converted } \mathrm{Kd} \text { to } \mathrm{mL} / \mathrm{g} \text {, reported as } \\
100 \mathrm{~m} 3 / \mathrm{kg}\end{array}$ & $\begin{array}{l}\text { McKinley } \\
\text { (1993) }\end{array}$ \\
\hline
\end{tabular}


TABLE 2-4 (Cont.)

\begin{tabular}{|c|c|c|c|c|c|c|c|}
\hline$\cdots$ & $\begin{array}{l}\text { Conc. of } \\
\text { Element }\end{array}$ & $\begin{array}{l}\mathrm{K}_{\mathrm{d}} \text { in } \mathrm{mL} / \mathrm{g} \\
\text { (or other } \\
\text { units or } \\
\% \text { sorbed) }\end{array}$ & $\begin{array}{l}\text { CEC } \\
\text { (meq/ } \\
100 \mathrm{~g}) \\
\end{array}$ & $\begin{array}{c}\text { BET Area } \\
\left(\mathrm{m}^{2} / \mathrm{g}\right) \text { or Size } \\
(\text { microns }) \\
\end{array}$ & Solution Conditions & Notes & Reference \\
\hline bentonite & trace & $100-5,000$ & & fine powder & $\begin{array}{l}\mathrm{pH}=3-8, \\
\text { bentonite: water }=1: 50 \text { to } \\
1: 400,[\mathrm{Eu}] \text { or }[\mathrm{Cs}+\mathrm{Sr}]=1 \mathrm{e}- \\
2 \text { to } 1 \mathrm{e}-8 \mathrm{M}, \mathrm{BW} \text { water } \\
\text { analysis }(\mathrm{ug} / \mathrm{mL}) \text { : } \\
\mathrm{Na}+=1.4 \mathrm{e} 3, \mathrm{Mg} 2+=97, \\
\mathrm{Ca} 2+=77, \mathrm{~K}+=42, \mathrm{Cl}-=1 \mathrm{e} 3 \text {, } \\
\mathrm{CO} 32-=\text { high, etc }\end{array}$ & $\begin{array}{l}\text { bentonite characterized as predominantly } \\
\text { montmorillonite; } \mathrm{pH}<4 \text { no hydrolysis of } \\
\text { Am or sorption }\end{array}$ & $\begin{array}{l}\text { Murali } \\
(2002)\end{array}$ \\
\hline crushed YMP tuff & 7e-9M & 1000 & & $\begin{array}{l}75 \text { to } 100 \\
\text { microns }\end{array}$ & $\begin{array}{l}\mathrm{J}-13 \text { well water from NTS, } \\
3 \mathrm{e}-3 \mathrm{M} \text { NaHCO3; column } \\
\text { tests at } 0.15 \mathrm{~mL} / \mathrm{h} \text { for } \\
\text { column density of } 1 \mathrm{~g} / \mathrm{mL}\end{array}$ & typically $1 \%$ breakthru; & $\begin{array}{l}\text { Thompson } \\
\text { (1989) }\end{array}$ \\
\hline basalt (igneous rock) & $2 \mathrm{e}-9 \mathrm{M}$ & $2,000-6,000$ & & & $\begin{array}{l}\text { synthetic groundwater, } \\
\mathrm{pH}=7-8\end{array}$ & & $\begin{array}{l}\text { Triay } \\
(1991)\end{array}$ \\
\hline $\begin{array}{l}\text { clinoptilolite } \\
\left(\mathrm{M}_{8} \mathrm{Al}_{8} \mathrm{Si}_{28} \mathrm{O}_{27} 24 \mathrm{H}_{2} \mathrm{O},\right. \\
\text { natural zeolite from } \\
\text { Castle Creek, purified } \\
\text { form: } 98 \% \text { clinoptilolite, } \\
2 \% \text { smectite and quartz) }\end{array}$ & $1 \mathrm{e}-12 \mathrm{M}$ & 4,900 & 100 & $75-500$ um & $\begin{array}{l}\mathrm{pH} 7.5 \text { buffer (sodium } \\
\text { carbonate-sodium } \\
\text { bicarbonate), } \mathrm{t}=21 \mathrm{~d} \text {, } \\
\mathrm{V} / \mathrm{m}=100 \mathrm{~mL} / \mathrm{g}\end{array}$ & reported CEC as $\sim 1 \mathrm{meq} / \mathrm{g}$ & $\begin{array}{l}\text { Triay } \\
(1991)\end{array}$ \\
\hline granite (igneous rock) & $2 \mathrm{e}-9 \mathrm{M}$ & $4,000-7,000$ & & & $\begin{array}{l}\text { synthetic groundwater, } \\
\mathrm{pH}=7-8\end{array}$ & & $\begin{array}{l}\text { Triay } \\
(1991)\end{array}$ \\
\hline $\begin{array}{l}\text { romanechite (manganese } \\
\text { oxyhydroxide, } \\
\mathrm{BaMn}^{2+} \mathrm{Mn}_{8}{ }^{4+} \mathrm{O}_{16}(\mathrm{OH})_{4}, \\
\text { from Casa Grande, } \mathrm{AZ} \text { ) }\end{array}$ & $1 \mathrm{e}-12 \mathrm{M}$ & 330,000 & & $75-500$ um & $\begin{array}{l}\mathrm{pH} 7.5 \text { buffer (sodium } \\
\text { carbonate-sodium } \\
\text { bicarbonate), } \mathrm{t}=21 \mathrm{~d} \text {, } \\
\mathrm{V} / \mathrm{m}=100 \mathrm{~mL} / \mathrm{g}\end{array}$ & & $\begin{array}{l}\text { Triay } \\
(1991)\end{array}$ \\
\hline
\end{tabular}


TABLE 2-4 (Cont.)

\begin{tabular}{|c|c|c|c|c|c|c|c|}
\hline Sorbent Material & $\begin{array}{l}\text { Conc. of } \\
\text { Element }\end{array}$ & $\begin{array}{l}\mathrm{K}_{\mathrm{d}} \text { in } \mathrm{mL} / \mathrm{g} \\
\text { (or other } \\
\text { units or } \\
\% \text { sorbed) } \\
\end{array}$ & $\begin{array}{l}\text { CEC } \\
(\mathrm{meq} / \\
100 \mathrm{~g}) \\
\end{array}$ & $\begin{array}{c}\text { BET Area } \\
\left(\mathrm{m}^{2} / \mathrm{g}\right) \text { or Size } \\
(\text { microns })\end{array}$ & Solution Conditions & Notes & Reference \\
\hline $\begin{array}{l}\text { YMP tuff (G4-1502, } \\
71 \% \text { clinoptilolite, } 15 \% \\
\text { opal-CT, } 4 \% \text { quartz, } 3 \% \\
\text { alkali feldspar, } 2 \% \\
\text { smectite) }\end{array}$ & $1 \mathrm{e}-8 \mathrm{M}$ & 2,000 & & & $\mathrm{~J}-13$ groundwater, $\mathrm{pH}=7-8$ & & $\begin{array}{l}\text { Triay } \\
(1991)\end{array}$ \\
\hline $\begin{array}{l}\text { YMP tuff (GU3-433, } \\
76 \% \text { alkali feldspar, } \\
15 \% \text { cristobalite, } 2 \% \\
\text { mica, } 1 \% \text { hematite) }\end{array}$ & $2 \mathrm{e}-7 \mathrm{M}$ & 30,000 & & & $\mathrm{~J}-13$ groundwater, $\mathrm{pH}=7-8$ & & $\begin{array}{l}\text { Triay } \\
(1991)\end{array}$ \\
\hline $\begin{array}{l}\text { YMP tuff (GU3-688, } \\
68 \% \text { alkali feldspar, } \\
27 \% \text { cristobalite, } 4 \% \\
\text { quartz, } 1 \% \text { smectite) }\end{array}$ & $1 \mathrm{e}-12 \mathrm{M}$ & 12,000 & & $75-500$ um & $\begin{array}{l}\mathrm{J}-13 \text { groundwater, } \mathrm{pH}=7-8 \text {, } \\
\text { alkalinity }=1 \mathrm{mN}\left(\mathrm{HCO}_{3}{ }^{-}\right) \\
\mathrm{t}=21 \mathrm{~d}, \mathrm{~V} / \mathrm{m}=100 \mathrm{~mL} / \mathrm{g}\end{array}$ & & $\begin{array}{l}\text { Triay } \\
(1991)\end{array}$ \\
\hline layered metal sulfides & & & & & & $\begin{array}{l}\text { Manos (2009) indicates that clays } \\
\text { composed of aluminosilicate anionic slabs } \\
\text { with alkali or alkaline earth counter cations } \\
\text { are excellent cationic exchangers for range } \\
\text { of inorganic ions (ref 1-13) }\end{array}$ & \\
\hline Wellenberg colloids & $1.5 \mathrm{e}-5 \mathrm{M}$ & $\begin{array}{l}60,000- \\
400,000\end{array}$ & & & $\begin{array}{l}\text { (a) } 2 \mathrm{e}-2 \mathrm{M} \mathrm{NaHCO} 3, \mathrm{pH} 8.6 \\
\text { and (b) } 0.02 \mathrm{M} \mathrm{NaCl}, 1 \mathrm{e}-5 \mathrm{M} \\
\mathrm{HCO} 3, \mathrm{pH} 8.6\end{array}$ & & \\
\hline
\end{tabular}

\section{TABLE 2-4 Notes and citations:}

1. Penrose et al. (1990) reported that plutonium and americium were associated with siliceous colloids in an alluvial aquifer at Los Alamos National Laboratory. W. R. Penrose, et al. (1990), "Mobility of plutonium and americium through a shallow aquifer in a semiarid region," Environ. Sci. Technol. 24(2), 228-234 


\section{TABLE 2-4 Notes and citations (Cont.):}

2. At the Nevada Test Site, transition metals and lanthanide radionuclides associated with inorganic colloids were recovered from groundwater $300 \mathrm{~m}$ from a nuclear detonation cavity (Buddemeier and Hunt 1988). Americium and lead were transported in the vadose zone with organic and inorganic colloids. R.W Buddemeier and J.R Hunt (1988), "Transport of colloidal contaminants in groundwater: radionuclide migration at the Nevada Test Site," Appl. Geochem., 3, 535-548.

3. Under most geologic conditions, transport of radionuclides via iron oxide or clay colloids dominates over transport of radionuclides by true polymerized radiocolloids (McCarthy and Zachara 1989). J.F McCarthy and J.M Zachara (1989), "Subsurface transports of contaminants," Environ. Sci. Technol. 23(5), 496-502.

4. Generation and mobilization of colloids have become an important issue for contaminant transport (Kersting et al. 1999), particularly facilitated transport of radionuclides as intrinsic colloids, for example, colloidal $\mathrm{Pu}(\mathrm{V})$ or as radionuclide-bearing pseudocolloids such as ${ }^{239} \mathrm{Pu},{ }^{237} \mathrm{~Np}$, ${ }^{243} \mathrm{Am}$, and ${ }^{247} \mathrm{Cm}$ from highlevel radioactive wastes or ${ }^{137} \mathrm{Cs},{ }^{90} \mathrm{Sr}$, and ${ }^{60} \mathrm{Co}$ from low-level radioactive wastes (Triay et al. 1996; Buddemeier and Hunt 1988; Kaplan et al. 1994;

McCarthy and Degueldre 1993). A.B. Kersting, et al. (1996), "Migration of plutonium in ground water at the Nevada Test Site," Nature 397, 56-59; I. Triay, C. Degueldre, A. Wistrom, C. Cotter, and W. Lemos (1996), "Progress report on colloid-facilitated transport at Yucca Mountain," Los Alamos report LA12959-MS; D.I Kaplan, P.M Bertsch, D.C Adriano, and K.A Orlandini (1994), "Actinide association with groundwaters colloids in a coastal plain aquifer," Radiochim. Acta 66/67, 181-187; J. F. McCarthy and C. Degueldre, (1993), in Environmental Particles 2 (eds., J. Buffle and H.P. van Leeuwen), Ann Arbor, Lewis, pp. 247-315.

5. Triay et al. (1996) studied the generation and stability of colloids in Yucca Mountain groundwater and found that colloids of clays, silicon, and iron oxides were generated in the groundwater and irreversible sorption of radionuclides onto these colloids occurred.

6. Conca (2000) authored the analysis/modeling reports published by the Yucca Mountain Project of DOE that provide data for Am on colloids of hematite, goethite, montmorillonite, and silica in natural and synthetic groundwater. J. Conca, "Unsaturated zone and saturated zone Transport properties (U0100)," UNT Digital Library, Las Vegas, Nevada, http://digital.library.unt.edu/ark:/67531/metadc782188, accessed October 3, 2016.

7. Al-Attar (2003), "Uptake of RNs on microporous and layered ion exchange materials," J. Materials Chem., 13, $2963-2968$.

8. Allard (1984), "Mechanisms for the interaction of AmIII and NpV with geologic media," Mat. Res. Soc. Symp. Proc., 26, 899-906.

9. Lujaniene et al. (2009), “Application of inorganic sorbents for removal of Cs Sr Pu and Am from contaminated solutions,” J. Radioanl. Nucl. Chem. 282, 787-791.

10. Lujaniene et al. (2010), "Effect of natural clay components on sorption of Cs Pu and Am by the clay," J. Radioanal. Nucl. Chem. 286, 353-359.

11. Marsh et al. (1995), "Effects of soluble organic complexants and their degradation products on the removal of selected radionuclides from high level waste, Part II: Distribution of Sr, Cs, Tc, and Am onto 32 absorbers from four variations of Hanford Tank 101-SY simulant solution," Los Alamos National Lab Report, LA-12943, April 1995, 1-47.

12. Marsh et al. (1995), "Effects of soluble organic complexants and their degradation products on the removal of selected radionuclides from high level waste, Part III: Distribution of Sr, Cs, Tc, and Am onto 33 absorbers from four variations of a 3:1 dilution of Hanford Complexant Concentrate (CC) simulant solution, Part IV: The effects of varying dilution ratios on the distributions of $\mathrm{Sr}, \mathrm{Cs}, \mathrm{Tc}, \mathrm{Pu}$, and Am onto 12 absorbers from Hanford Complexant Concentrate (CC) simulant solution,” Los Alamos National Lab Report, LA-13000, Sept. 1995, 1-64. 


\section{TABLE 2-4 Notes and citations (Cont.):}

13. McKinley and Scholtis (1993), “A comparison of radionuclide sorption databases used in recent performance assessments," J. Contam. Hydrol., 13, 347-363 (Table 3, data from Refs 1-6; Table 4, data from Ref. 9, Vieno and Suolanen, 1991).

14. Murali and Mathur (2002), "Sorption chars of Am, Sr, Cs on bentonite and granite," J. Radioanal. Nucl. Chem., 254(1), 129-136. (Refer to Refs 18 and 19 for Am complexing agents.)

15. Thompson (1989), J. Radioanal. Nucl. Chem. 130, 353-364; data from Refs. 1 and 2.

16. Allard and Beall (1979), J. Environ. Sci. Health A14, 507, Refs. 7 and 8; Allard et al. (1980), Nucl. Technol. $49,474$.

17. Triay et al. (1991), "Sorption of Am in tuff and pure minerals using synthetic and natural groundwaters," Radiochim. Acta, 52/53, 141-145.

18. Allard and Beall (1979), J. Environ. Sci. Health A14, 507, Refs. 7 and 8; Allard et al. (1980), Nucl. Technol. $49,474$.

19. Thomas (1987), Los Alamos Natl Lab, LA-10960-MS, Ref. 13. 
As much of the literature does not provide data on Am- and Cs-clay sorption properties with $\mathrm{K}^{+}$or $\mathrm{NH}_{4}{ }^{+}$present in our decontamination solution, we had to down-select the sorbents that might be expected to have a sufficiently high affinity and rapid uptake of Cs or Am in the SuperGel formulation. Based on our review of the literature, clays provide high partitioning coefficients and are abundant in nature, and thus meet important requirements for the decontamination technology, since abundant materials are available worldwide and they are costeffective. Transition metal oxide minerals (e.g., magnetite goethite, hematite, hydrous manganese, and stannic oxides) are not selective toward cesium at a high ionic strength due to competitive adsorption onto the charged surface sites. Many clays are similarly non-selective except at low-ionic strength (e.g., montmorillonite or bentonite). Illite and vermiculite, because of their very large $K_{d}$ values, are interesting. Zeolites have a higher selectivity than many sorbents due to their specific cage openings that aid selectivity by providing some physical discrimination as some ions can pass into the pores and others cannot. Chabazite and the synthetic crystalline silico-titanate are interesting. Soil sediments are interesting because of their immediate availability around the world but the studies focus on dilute groundwaters. Moreover, since transition metal oxides dominate the mineral surfaces within soils, there is good reason to believe that the $\mathrm{K}_{\mathrm{d}}$ values for $\mathrm{Cs}^{+}$will be low in high salt solutions. The presence of various clays within soils suggests that certain soils may be candidates for use as sequestering agents. But tests would need to be conducted to understand what types of soils would be suitable. The hexacyanoferrates are interesting due to their high $\mathrm{K}_{\mathrm{d}}$ at high-ionic strength. However, there is a question about its availability for immediate deployment in case of an emergency.

Similar to the data on cesium sorption, the experimental parameters used for the sorption experiments in the literature for $\mathrm{Am}^{3+}$ are limited or insufficiently defined. Moreover, the partitioning coefficients reported in the literature for many naturally occurring materials vary widely due to their heterogeneous composition (many mineral phases and adsorbed phases) and poor characterization of their composition. However, the individual, pure mineral phases in the naturally occurring clays offer predictable sorption behavior but may not be available in bulk quantities. Some materials such as synthetic antimony silicates, birnessite (a layered manganese oxide), and titanosilicates in $0.01 \mathrm{M} \mathrm{Ca}^{2+}$ or $\mathrm{Na}^{+}$and up to $1 \mathrm{M} \mathrm{H}^{+}$; romanechite (manganese oxyhydroxide), bentonite (montmorillonite), and smectites are reported to have high partitioning coefficients for americium $\left(\mathrm{K}_{\mathrm{d}} \sim 10^{3}-10^{7} \mathrm{~mL} / \mathrm{g}\right)$ in moderately salty waters and may warrant investigation if they can be readily available for large-scale use. Many sorbents were tested for sorption of $\mathrm{Am}^{3+}$ from Hanford nuclear waste tank solutions. Of these, cliniptilolite, synthetic titanates, and cyanoferrates performed well in the high ionic strength solutions. Soils and sediments from various regions around the world also sorbed $\mathrm{Am}^{3+}$ remarkably well. Sorption of $\mathrm{Am}^{3+}$ onto natural biotite (mica group phyllosilicate), calcium, aluminum, and transition metal oxides and hydroxides from groundwaters was good.

The companies that we contacted for availability of birnessite, cliniptilolite, illite, vermiculite, and romanechite are in Appendix 2. The suppliers for cliniptilolite and chabazite are in Appendix 3 and the bulk pricing for vermiculite concentrate is in Appendix 4.

The materials tests for cesium sorption were CST, KMS-2, illite, vermiculite, diatomaceous earth, and chabazite. 


\subsection{2 pH Dependence on Cesium Sequestering Agents}

We titrated the samples to qualitatively assess the buffering capabilities of the sorbents. Figure 2-1 shows the volume of acid needed to reach $\mathrm{pH} 4$. Each $20 \mu \mathrm{L}$ results in an input of sufficient protons to produce $0.02 \mathrm{M}^{\left[\mathrm{H}^{+}\right]}$in an unbuffered solution $\left(20 \mu \mathrm{L} \times 1 \mathrm{~mol} / 10^{6} \mu \mathrm{L}=\right.$ $2 \times 10^{-5}$ moles into a $1 \mathrm{~mL}$ solution, or $2 \times 10^{-2} \mathrm{moles} / \mathrm{L}$ or $\mathrm{pH}=1.7$ ). Note the strong buffering capabilities of KMS-2 and CST followed by vermiculite and illite.

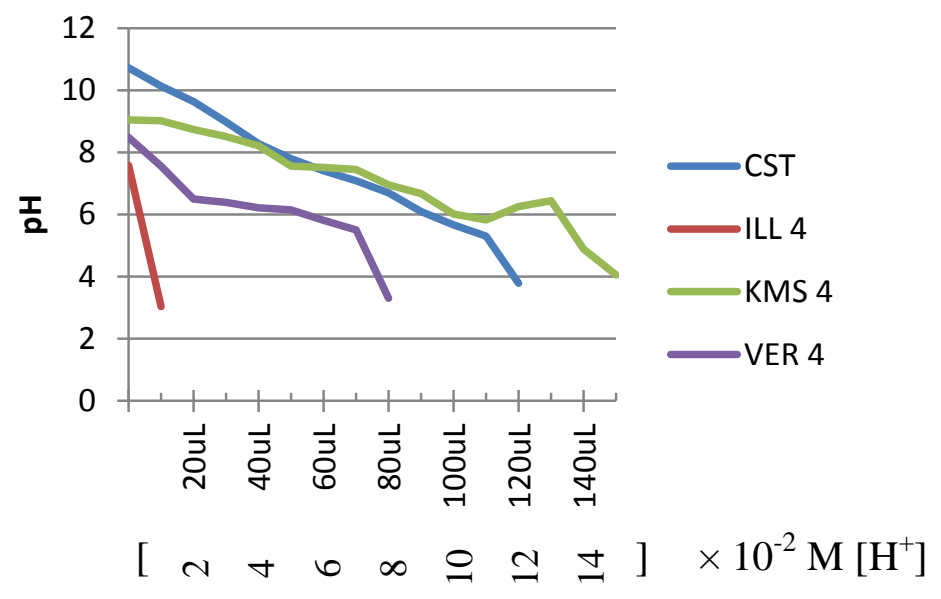

FIGURE 2-1 Volume of 1.0 $\mathrm{M} \mathrm{HNO}_{3}$ needed to adjust pH of unbuffered clay slurry $(10 \mathrm{mg} / \mathrm{mL}$ initially). The corresponding concentration of protons for an unbuffered solution is given in the bracketed abscissa labels.

\subsubsection{Kinetics of Cesium Sorption}

We completed experiments on the kinetic sorption of cesium onto various sorbents from an unbuffered solution (Figure 2-2). Even under mild agitation (rotary mixer), the sorption is at equilibrium within $10 \mathrm{~min}$. Of note, the sorption onto chabazite was very good at $5 \mathrm{~min}$ but decreased thereafter. 

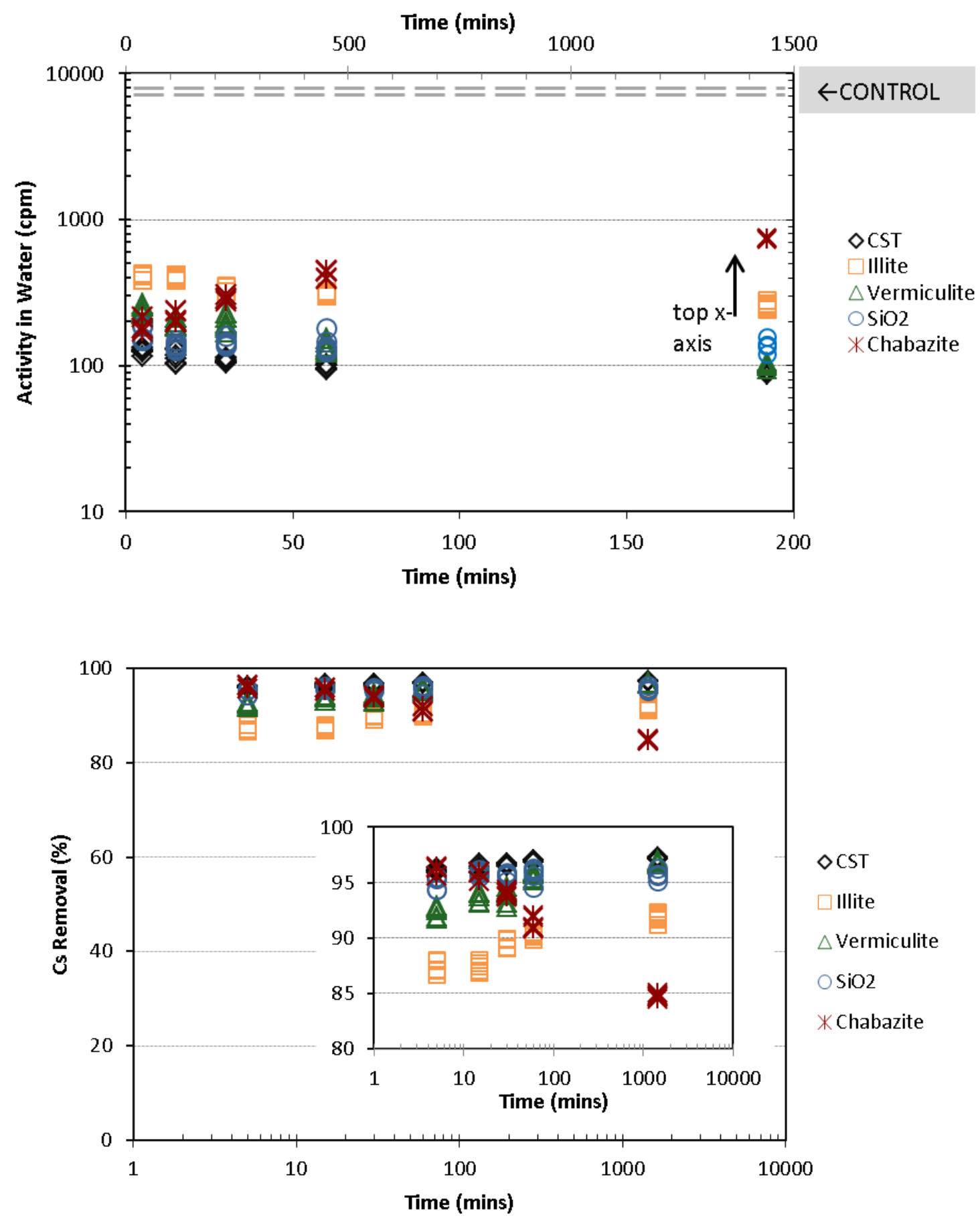

FIGURE 2-2 Sorption kinetics for ${ }^{137} \mathrm{Cs}$ in unbuffered water in terms of activity in the supernatant solution (top) and percent removal (bottom) of cesium from solution. 


\subsubsection{Sorption of Cesium in the Presence of Salt}

We next studied the sorption of cesium onto these materials while in the presence of various salts and reagents that are part of the SuperGel formulation. For these tests, the procedure was as described for the kinetic tests, except all samples were mixed for $1 \mathrm{~h}$ prior to sampling.

The results are displayed in Figures 2-3 to 2-6 in terms of activity in the supernatant solution, partitioning coefficient $\mathrm{K}_{\mathrm{d}}$, and percent removal of cesium from solution. Clear from the data is the strong negative effect that the salt concentration has on sorption at and above 0.1 M. The data indicate that CST is the best choice, and vermiculite is the next best choice overall. In the presence of $\mathrm{K}^{+}$, CST is clearly superior, with KMS and vermiculite giving comparable results. In the presence of $\mathrm{NH}_{4}^{+}$, vermiculite is best, followed by CST. CST is clearly better in $\mathrm{Na}_{2} \mathrm{CO}_{3}$ and HEDPA with vermiculite next best. These results beg the question of whether vermiculite would match the sorption of CST in carbonate and HEDPA if we converted them to their potassium form instead of the sodium and hydrogen forms. KMS-2 was removed from further testing because it was not deemed better than the other natural mineral choices.

\subsubsection{Sorption of Cesium with Mixed Isotope Hydrogel Formulation}

A combined SuperGel wash solution was formulated to contain potassium ions for cesium desorption and carbonate and diphosphonic acid for americium dissolution and chelation at concentrations of the ions and acid that allow optimal substrate decontamination while remaining compatible with the gel. We tested the sorption of cesium from $\mathrm{K}_{2} \mathrm{CO}_{3} / \mathrm{HEDPA}$ solutions onto montmorillonite, vermiculite, and chabazite. Results for sorption of Cs-137 on the natural materials follow the trend: chabazite $>$ vermiculite $>$ montmorillonite with an average $\mathrm{K}_{\mathrm{d}}$ of 32, 21, and $12 \mathrm{~mL} / \mathrm{g}$, respectively (refer to Figure 2-7). The relative standard deviations (RSDs) for the replicate tests and aliquots were low: $4.4 \%, 4.7 \%$, and $4.7 \%$, respectively, for the chabazite, vermiculite, and montmorillonite tests.

The sorption test results for chabazite with the combined cesium and americium decontamination solution formulation were compared with single-component solutions (Figure 2-8). The sorption of Cs-137 onto chabazite in the mixed formulation exhibits the same behavior as for the solutions of $\mathrm{KCl}$ or $\mathrm{NH}_{4} \mathrm{Cl}$ alone, as it is adversely affected by high concentrations of potassium or ammonium ions. For Cs-137 sorption onto chabazite in solutions containing $\mathrm{K}^{+}$ions, the sorption remains high up to $0.1 \mathrm{M} \mathrm{K}^{+}\left(\mathrm{K}_{\mathrm{d}}>300\right)$ and drops by a factor of 6 at $0.5 \mathrm{M} \mathrm{K}^{+}\left(\mathrm{K}_{\mathrm{d}}=51\right)$ and decreases by an additional factor of 1.5 at $1 \mathrm{M} \mathrm{K}^{+}$. At the $1 \mathrm{M} \mathrm{K}^{+}$ concentration, decontamination of the Cs-137 from the concrete would be more effective while still permitting effective capture or uptake by the sorbent. In contrast, the Cs-137 sorption behavior onto chabazite does not appear to be adversely affected by $\mathrm{Na}^{+}, \mathrm{CO}_{3}{ }^{2-}$, or HEDPA in solution concentrations of up to $0.5 \mathrm{M}\left(\mathrm{K}_{\mathrm{d}}>1000\right)$. 

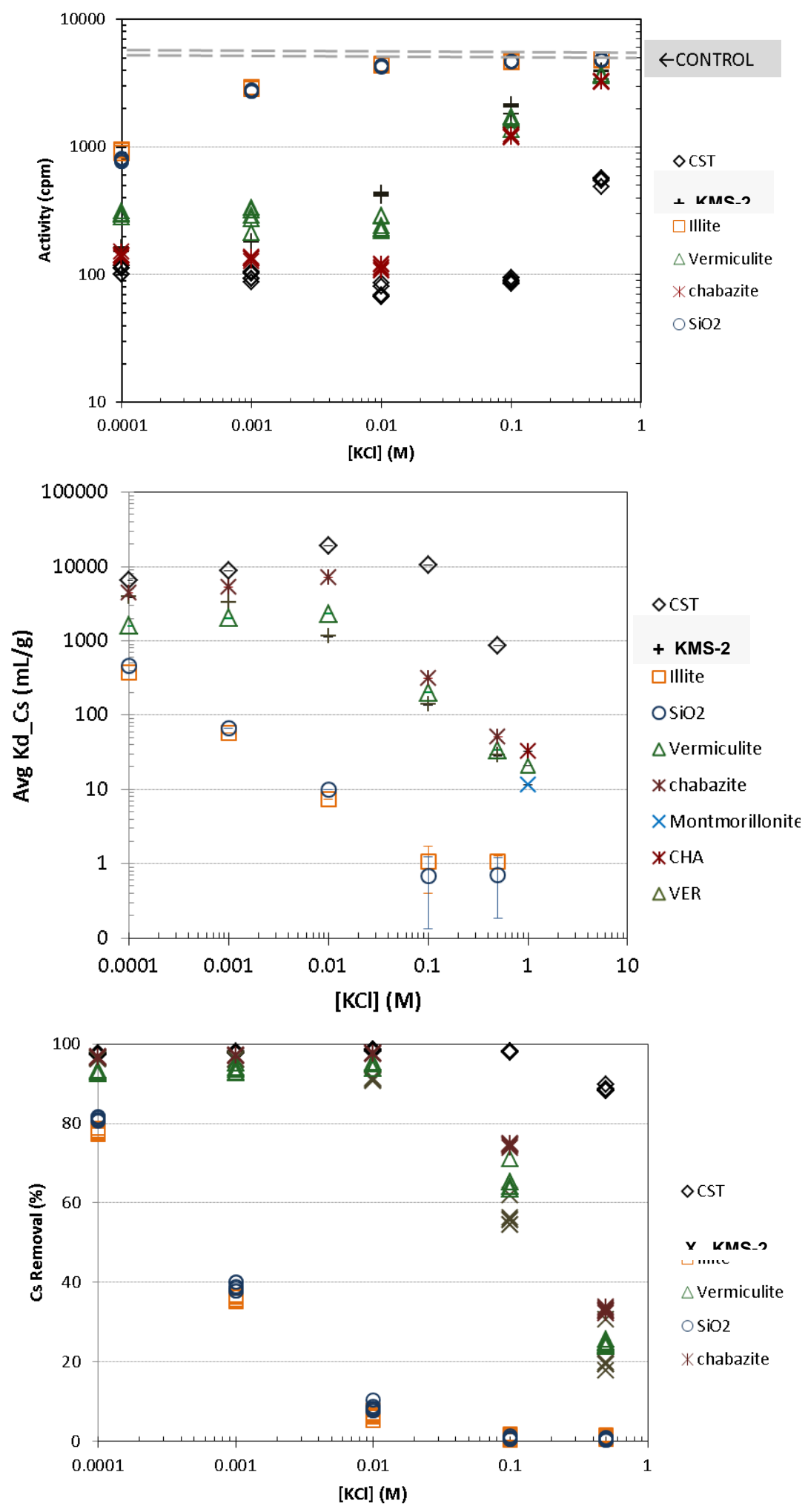

FIGURE 2-3 Effect of $\mathrm{KCl}$ on the equilibrium sorption of cesium onto various sequestering agents in terms of activity in the supernatant solution (top), partitioning coefficient $K_{d}$ (middle), and percent removal (bottom) of cesium from solution. 

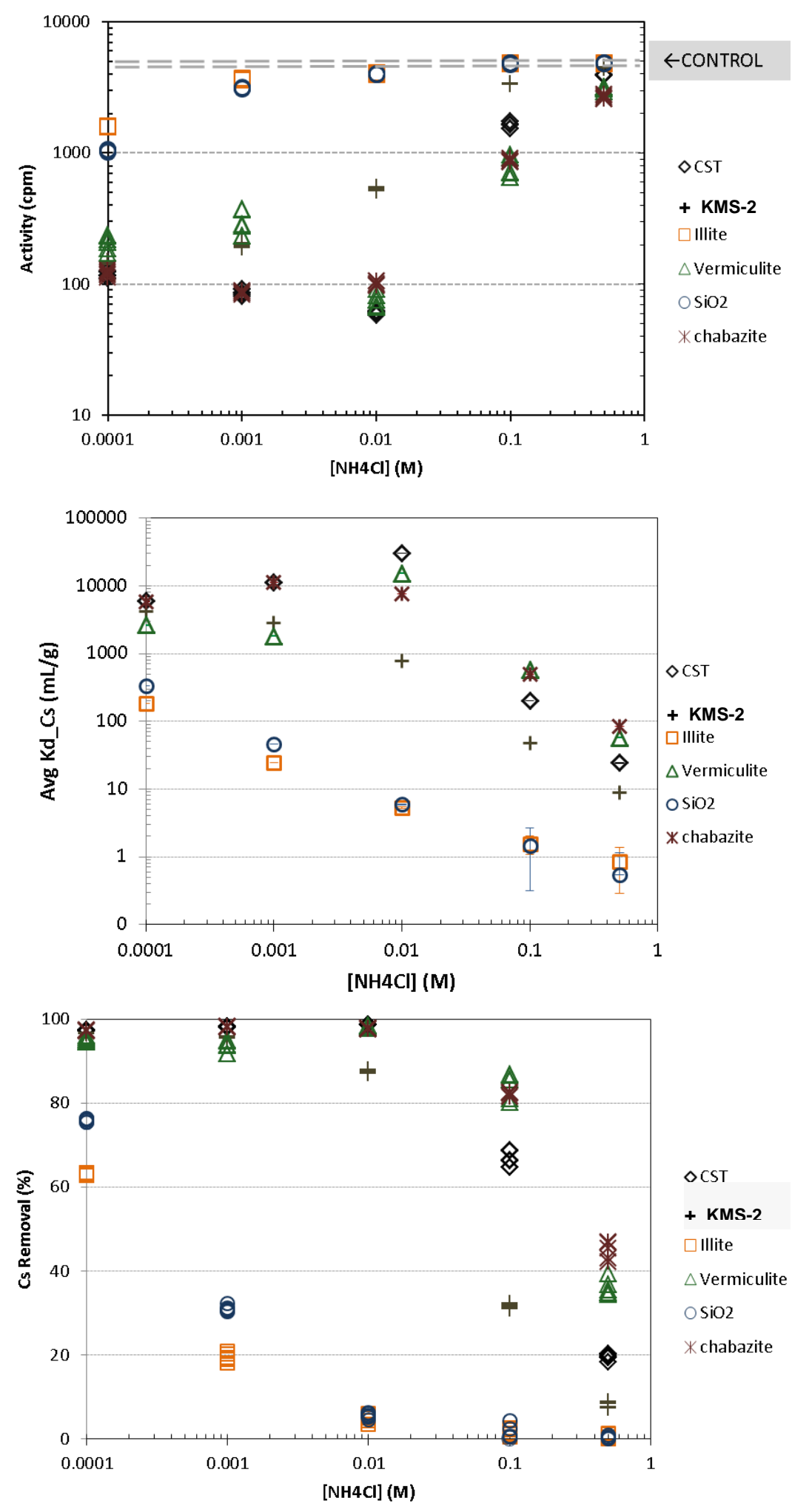

FIGURE 2-4 Effect of $\mathrm{NH}_{4} \mathrm{Cl}$ on the equilibrium sorption of cesium onto various sequestering agents in terms of activity in the supernatant solution (top), partitioning coefficient $K_{d}$ (middle), and percent removal (bottom) of cesium from solution. 


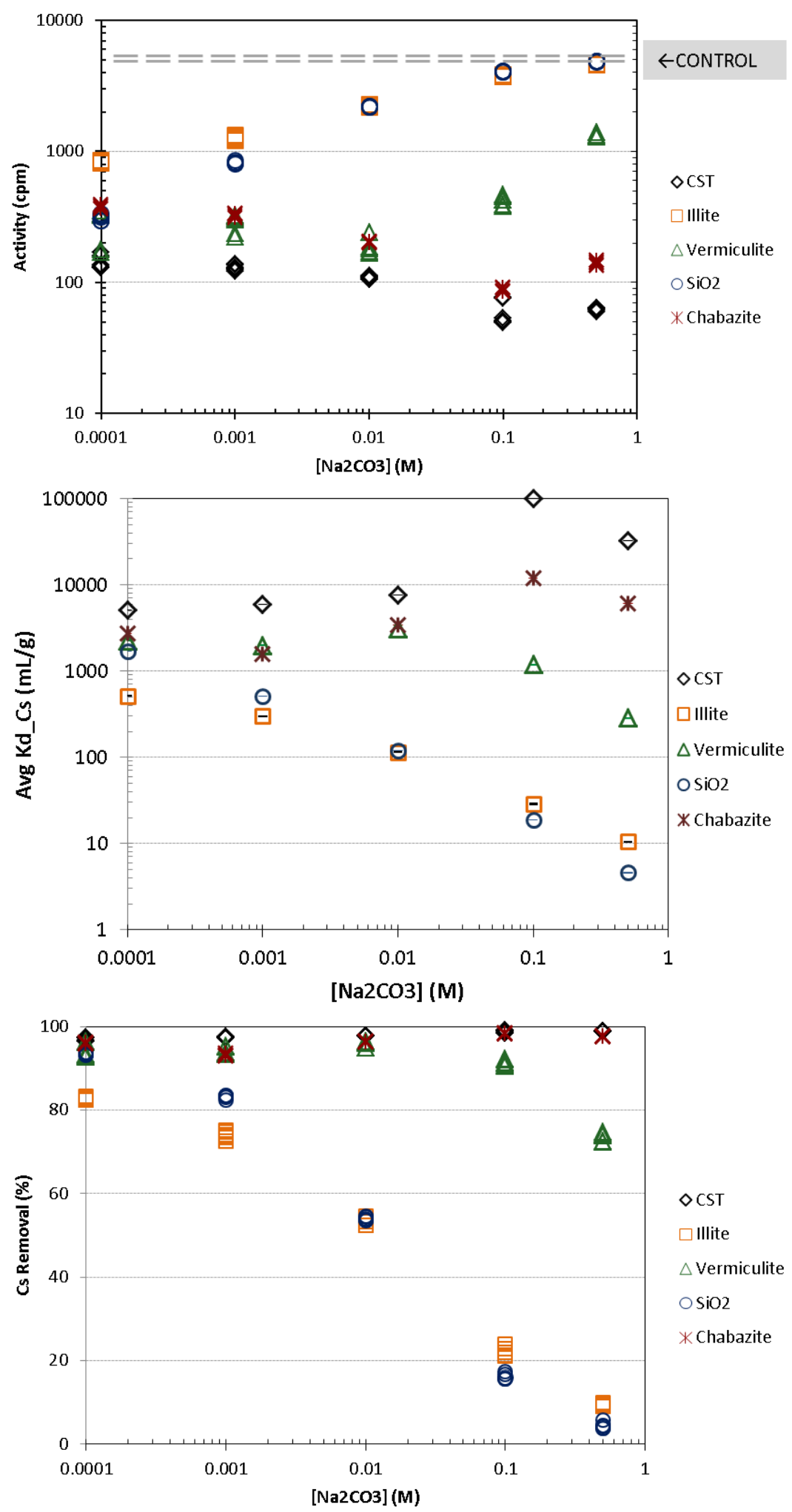

FIGURE 2-5 Effect of $\mathrm{Na}_{2} \mathrm{CO}_{3}$ on the equilibrium sorption of cesium onto various sequestering agents in terms of activity in the supernatant solution (top), partitioning coefficient $K_{d}$ (middle), and percent removal (bottom) of cesium from solution. 

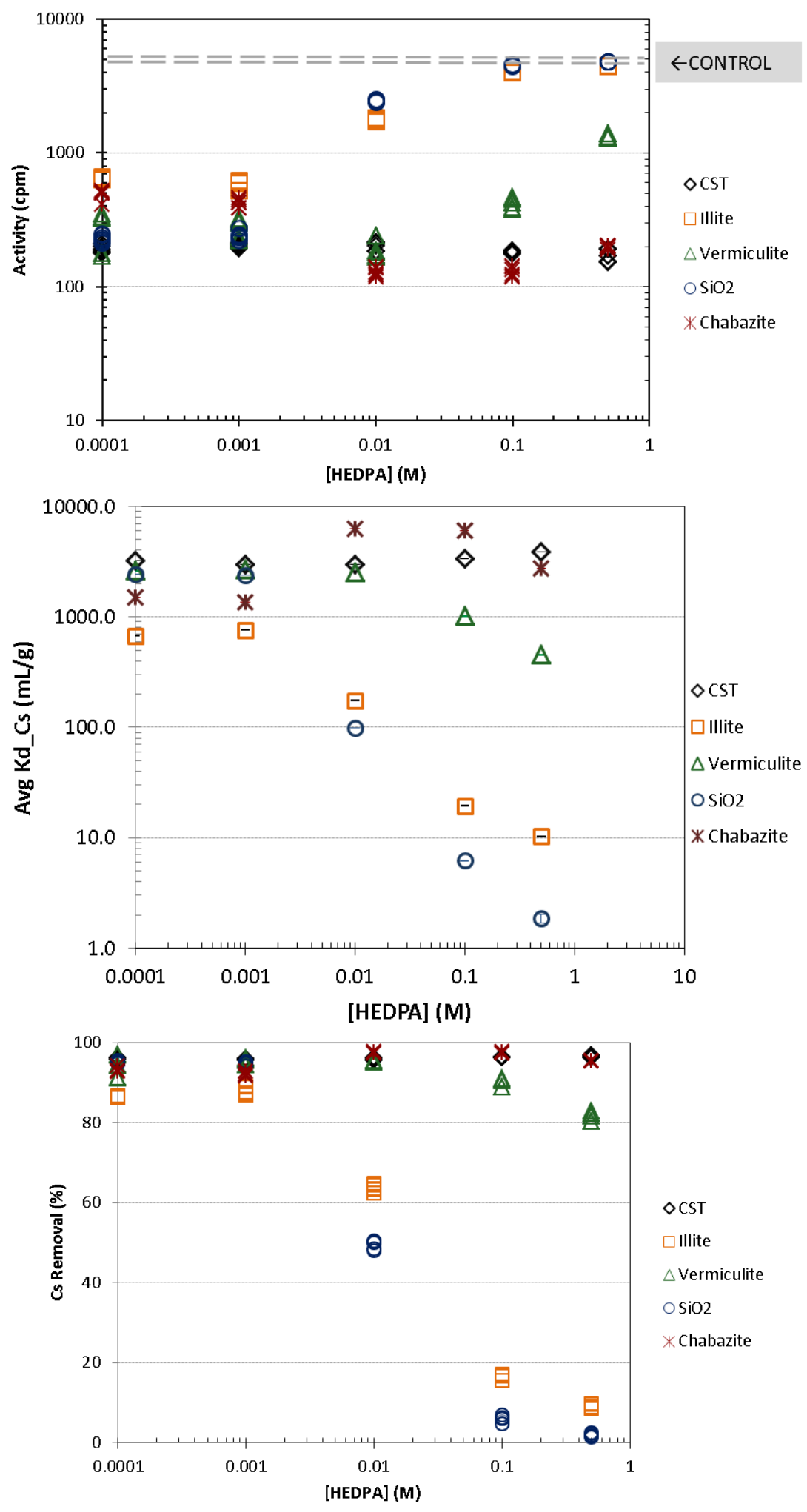

FIGURE 2-6 Effect of HEDPA on the equilibrium sorption of cesium onto various sequestering agents in terms of activity in the supernatant solution (top), partitioning coefficient $K_{d}$ (middle), and percent removal (bottom) of cesium from solution. 


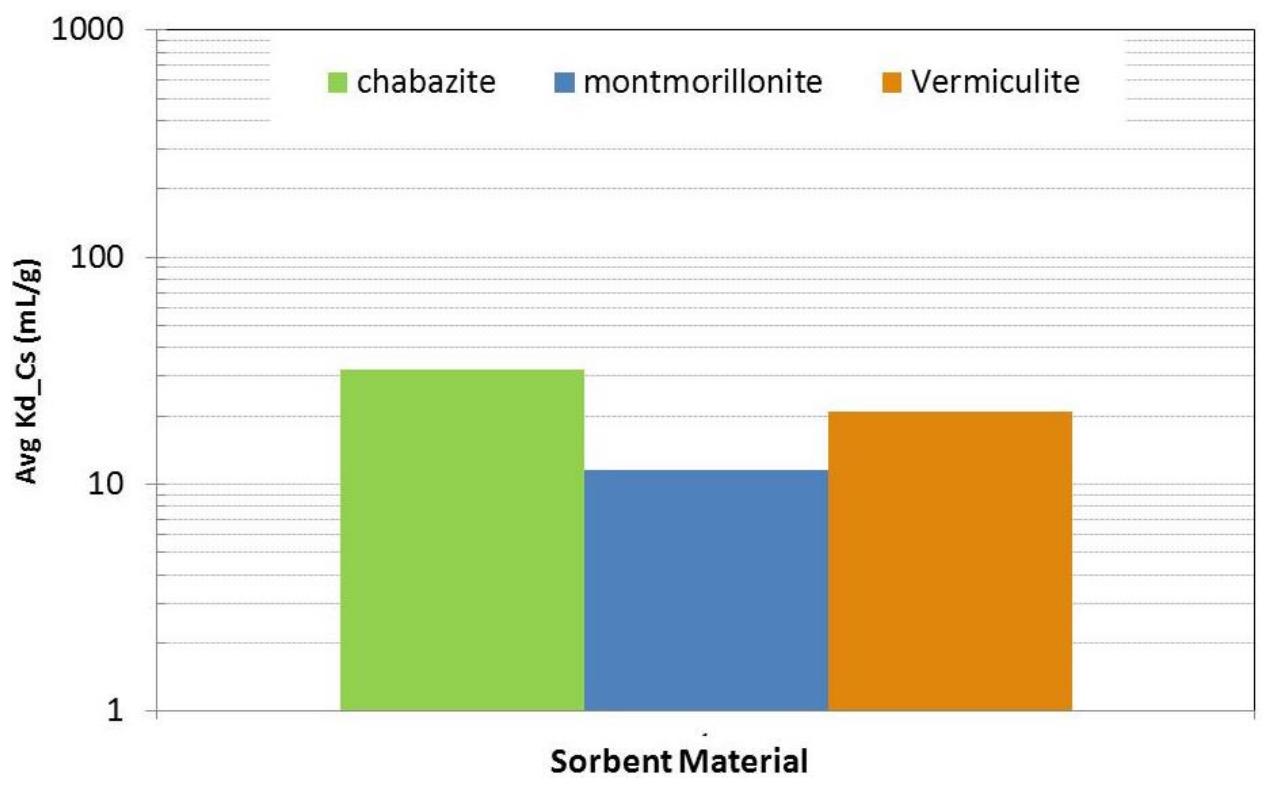

FIGURE 2-7 Sorption of Cs-137 onto clays from a solution of $1 \mathrm{M} \mathrm{K}^{+}$, $0.5 \mathrm{M} \mathrm{CO}_{3}{ }^{2-}$, and $0.025 \mathrm{M}$ HEDPA after $1 \mathrm{~h}$ of gentle agitation at $10 \mathrm{mg} / \mathrm{mL}$ slurry concentration.

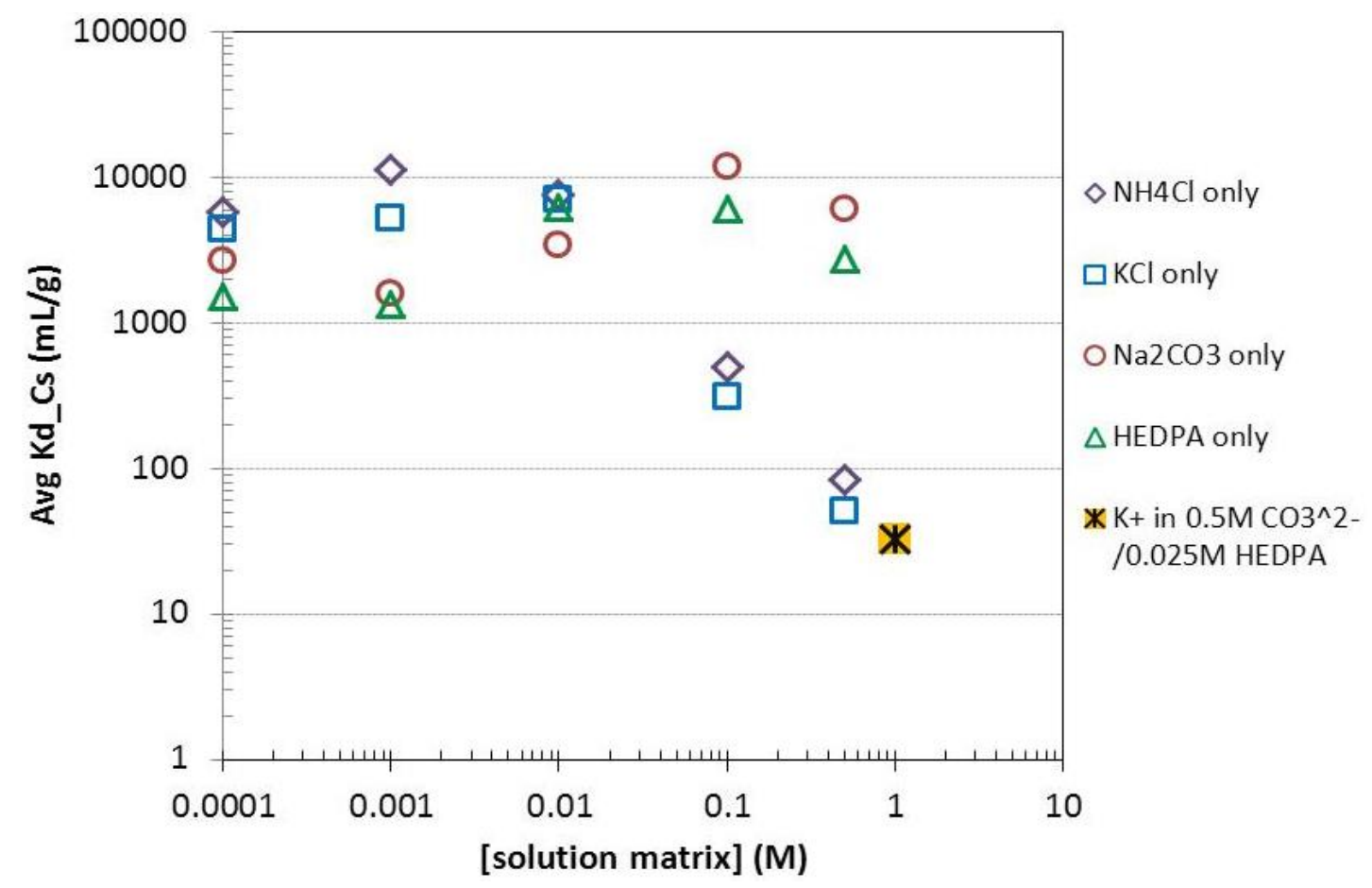

FIGURE 2-8 Sorption of Cs-137 onto chabazite from 0.0001-0.5 M solutions of $\mathrm{NH}_{4} \mathrm{Cl}, \mathrm{KCl}, \mathrm{Na}_{2} \mathrm{CO}_{3}$, and HEDPA or a combined solution of $1 \mathrm{M} \mathrm{K}^{+}, 0.5 \mathrm{M} \mathrm{CO}_{3}{ }^{2-}$, and $0.025 \mathrm{M}$ HEDPA. Tests were allowed to equilibrate for $1 \mathrm{~h}$ under gentle agitation at $10 \mathrm{mg} / \mathrm{mL}$ slurry concentrations. 
Results of Cs-137 sorption onto montmorillonite (Figure 2-9) compare the combined formulation solution of $1 \mathrm{M} \mathrm{K}^{+}, 0.5 \mathrm{M} \mathrm{CO}_{3}{ }^{2-}$, and $0.025 \mathrm{M}$ HEDPA with $\mathrm{NH}_{4} \mathrm{Cl}$ solutions that cover the concentration range of 0.0001 to $0.1 \mathrm{M}$. The low $\mathrm{K}_{\mathrm{d}}$ for Cs onto montmorillonite $\left(K_{d}=12\right)$ follows the trend for chabazite of decreasing $K_{d}$ with an increase in the ammonium ion.

The sorption test results for vermiculite with the combined cesium and americium decontamination formulation were compared with single-component solutions (Figure 2-10). For Cs-137 sorption onto vermiculite in solutions containing $\mathrm{K}^{+}$ions, the sorption remains high up to $0.1 \mathrm{M} \mathrm{K}^{+}\left(\mathrm{K}_{\mathrm{d}}>200\right)$ and drops by a factor of 6 at $0.5 \mathrm{M} \mathrm{K}^{+}\left(\mathrm{K}_{\mathrm{d}}=34\right)$ and decreases by an additional factor of 1.6 at $1 \mathrm{M} \mathrm{K}^{+}$. When comparing the $\mathrm{K}_{\mathrm{d}}$ 's from $0.5 \mathrm{M} \mathrm{Na}_{2} \mathrm{CO}_{3}$ solution to the mixed formulation of $0.5 \mathrm{M} \mathrm{K}_{2} \mathrm{CO}_{3}+0.025 \mathrm{M}$ HEDPA solution sorption tests, we found that the $\mathrm{K}_{\mathrm{d}}$ of 300 for $\mathrm{Cs}$ in $0.5 \mathrm{M} \mathrm{Na}_{2} \mathrm{CO}_{3}$ is much higher than that for the mixed formulation solution $\left(\mathrm{K}_{\mathrm{d}}=21\right)$, indicating that the $\mathrm{Na}^{+}$is less competitive with cesium for sorption sites than $\mathrm{K}+$ or $\mathrm{NH}_{4}{ }^{+}$.

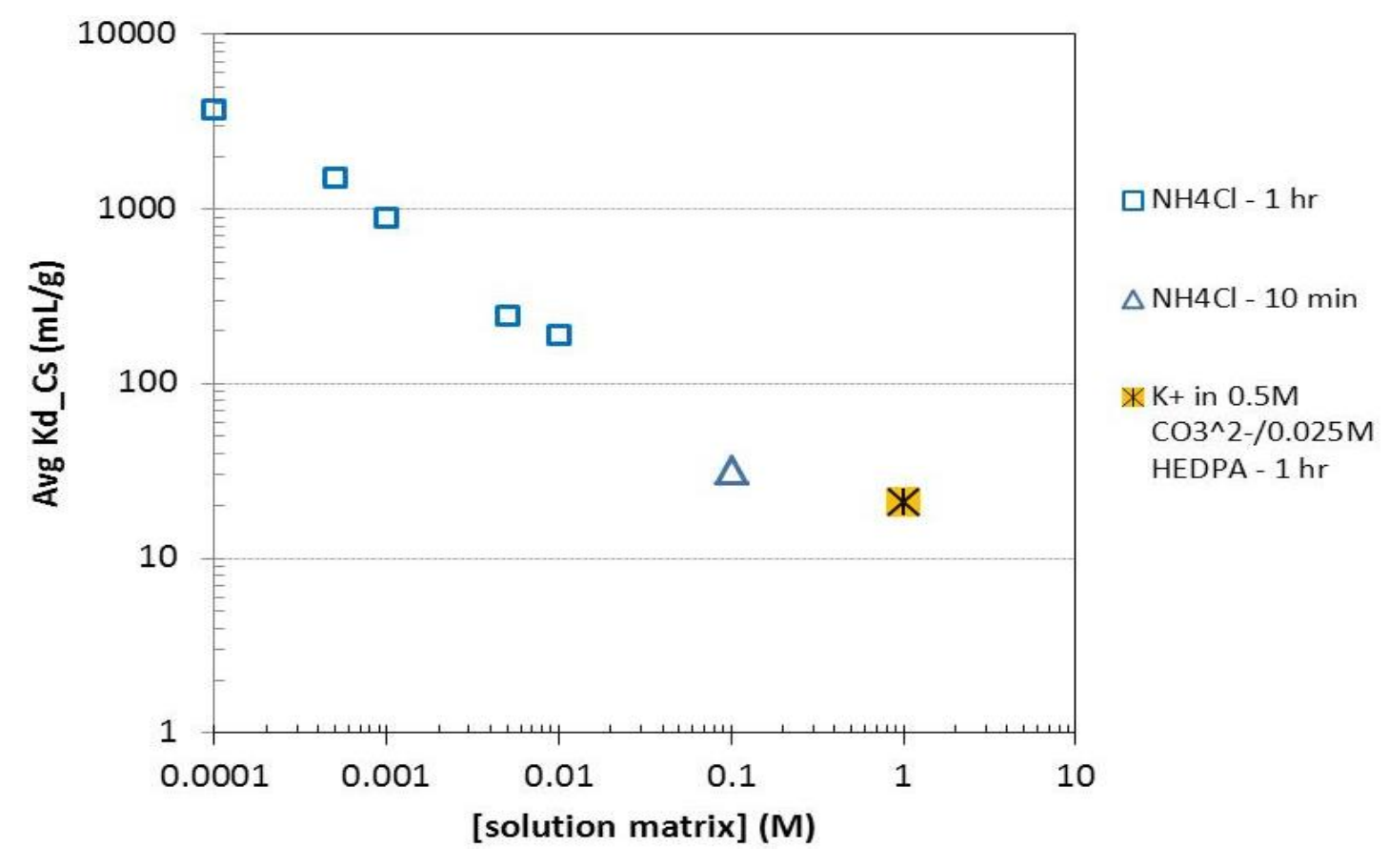

FIGURE 2-9 Sorption of Cs-137 onto montmorillonite from 0.0001-0.1 M solutions of $\mathrm{NH}_{4} \mathrm{Cl}$ or a combined formulation solution of $1 \mathrm{M} \mathrm{K}^{+}, 0.5 \mathrm{M} \mathrm{CO}_{3}{ }^{2-}$, and $0.025 \mathrm{M}$ HEDPA. Tests were allowed to equilibrate for $10 \mathrm{~min}$ or $1 \mathrm{~h}$ under gentle agitation at $10 \mathrm{mg} / \mathrm{mL}$ slurry concentrations. 


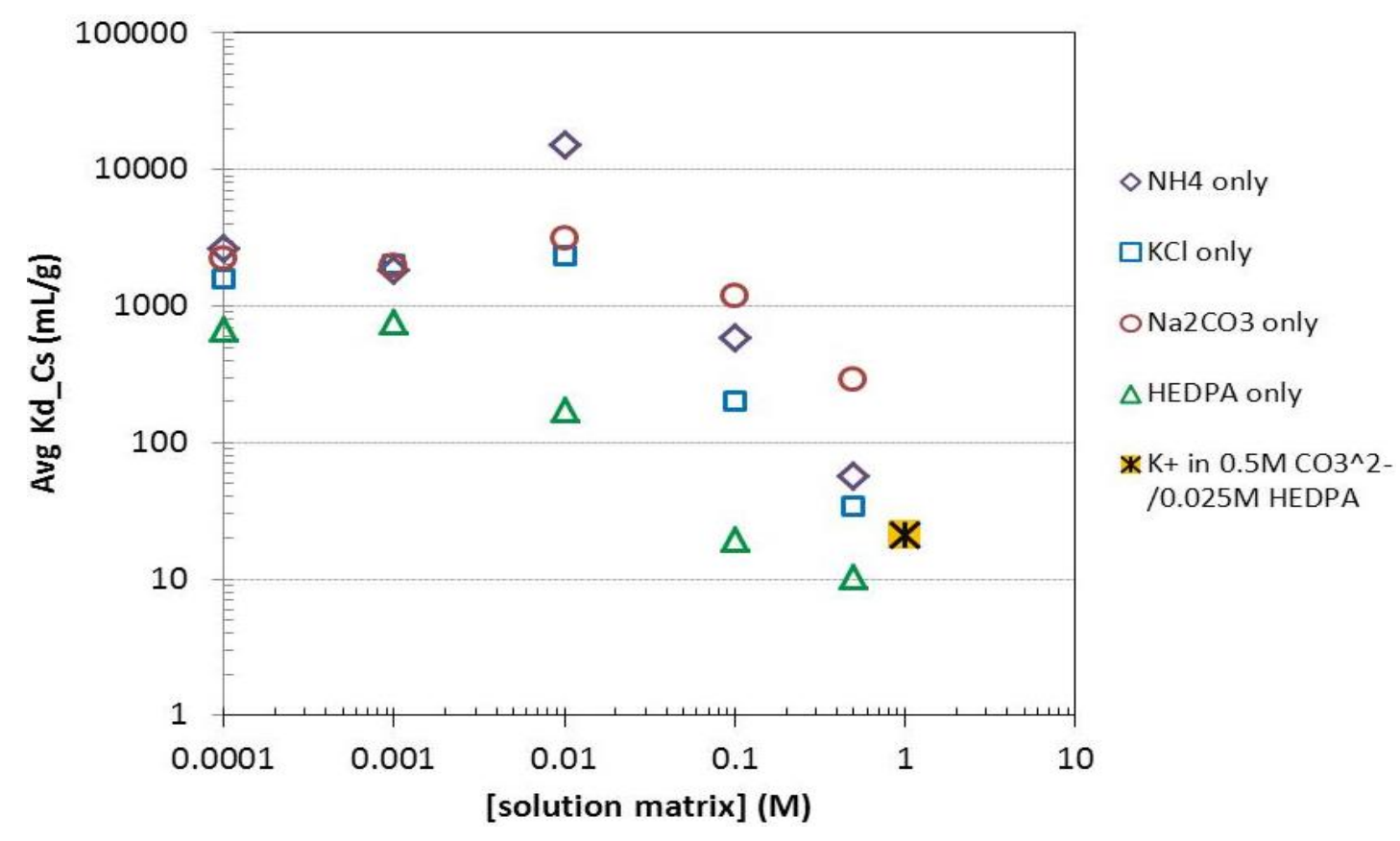

FIGURE 2-10 Sorption of Cs-137 onto vermiculite from 0.0001-0.5 M solutions of $\mathrm{NH}_{4} \mathrm{Cl}, \mathrm{KCl}, \mathrm{Na}_{2} \mathrm{CO}_{3}$, and HEDPA or a combined solution of $1 \mathrm{M} \mathrm{K}^{+}, 0.5 \mathrm{M} \mathrm{CO}_{3}{ }^{2-}$, and $0.025 \mathrm{M}$ HEDPA. Tests were allowed to equilibrate for $1 \mathrm{~h}$ under gentle agitation at $10 \mathrm{mg} / \mathrm{mL}$ slurry concentrations.

\subsubsection{Sorption of Cesium as a Function of Solid-to-Liquid Ratio}

We completed tests of the $\mathrm{K}_{\mathrm{d}}$ as a function of the sorbent clay slurry concentration and type of sorbent. Sorption was in the presence of $0.5 \mathrm{M} \mathrm{KCl}$ or $\mathrm{NH}_{4} \mathrm{Cl}$ using $10-125 \mathrm{mg}$ sorbent $/ \mathrm{mL}$ salt solution after mixing for 10 and $30 \mathrm{~min}$ (triplicate tests and duplicate aliquots for analysis) in a rotisserie mixer. For $\mathrm{KCl}$ (Figure 2-11), the $\mathrm{K}_{\mathrm{d}}$ 's are low for all materials, with montmorillonite performing better than vermiculite. However, for $\mathrm{NH}_{4} \mathrm{Cl}$ (Figure 2-12), the $\mathrm{K}_{\mathrm{d}}$ 's are much higher, with vermiculite performing much better than montmorillonite. 


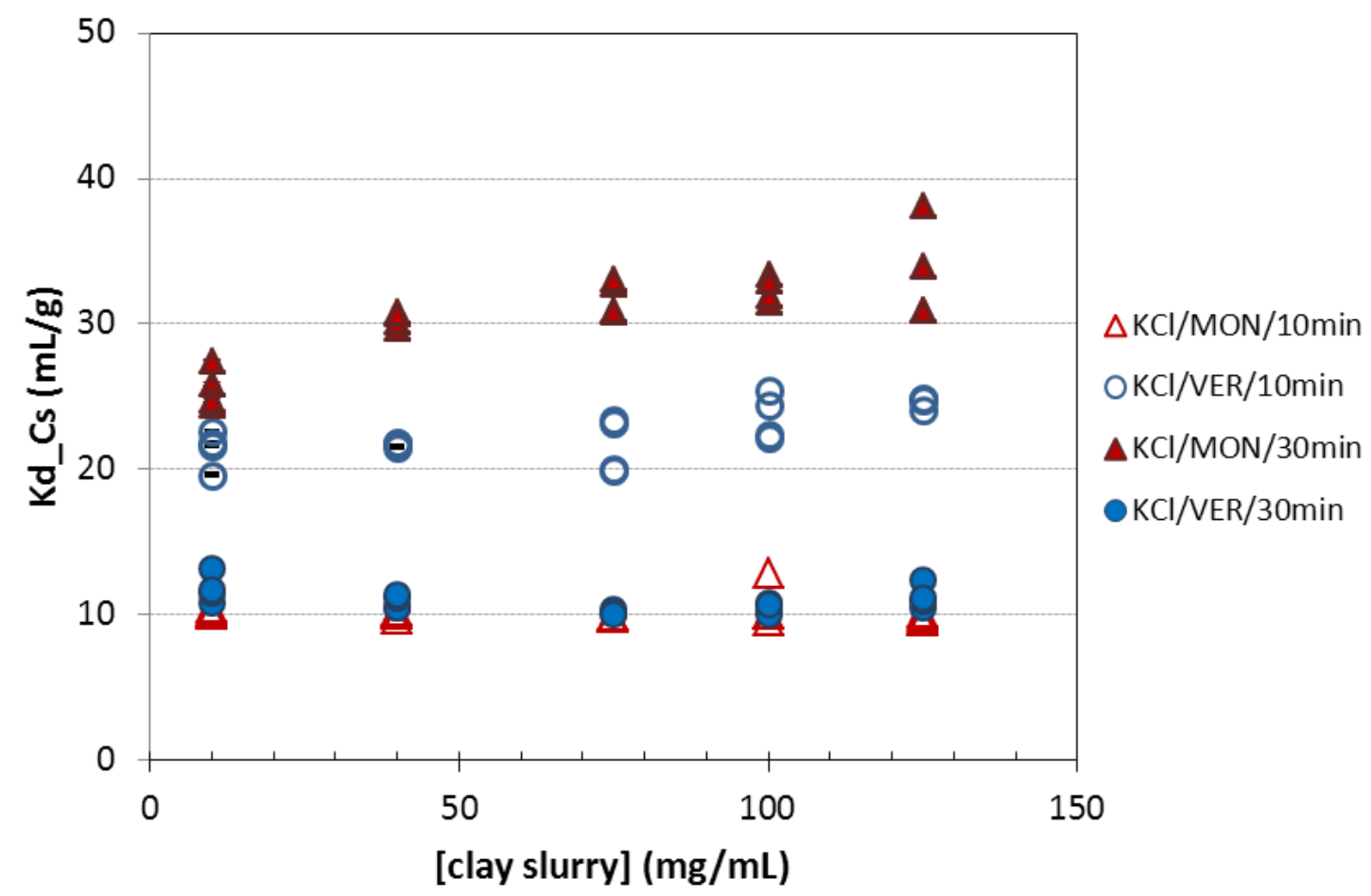

FIGURE 2-11 Sorption of Cs-137 onto clays from solution of $0.5 \mathrm{M} \mathrm{KCl}$ after $10 \mathrm{~min}$ and $30 \mathrm{~min}$ of gentle agitation.

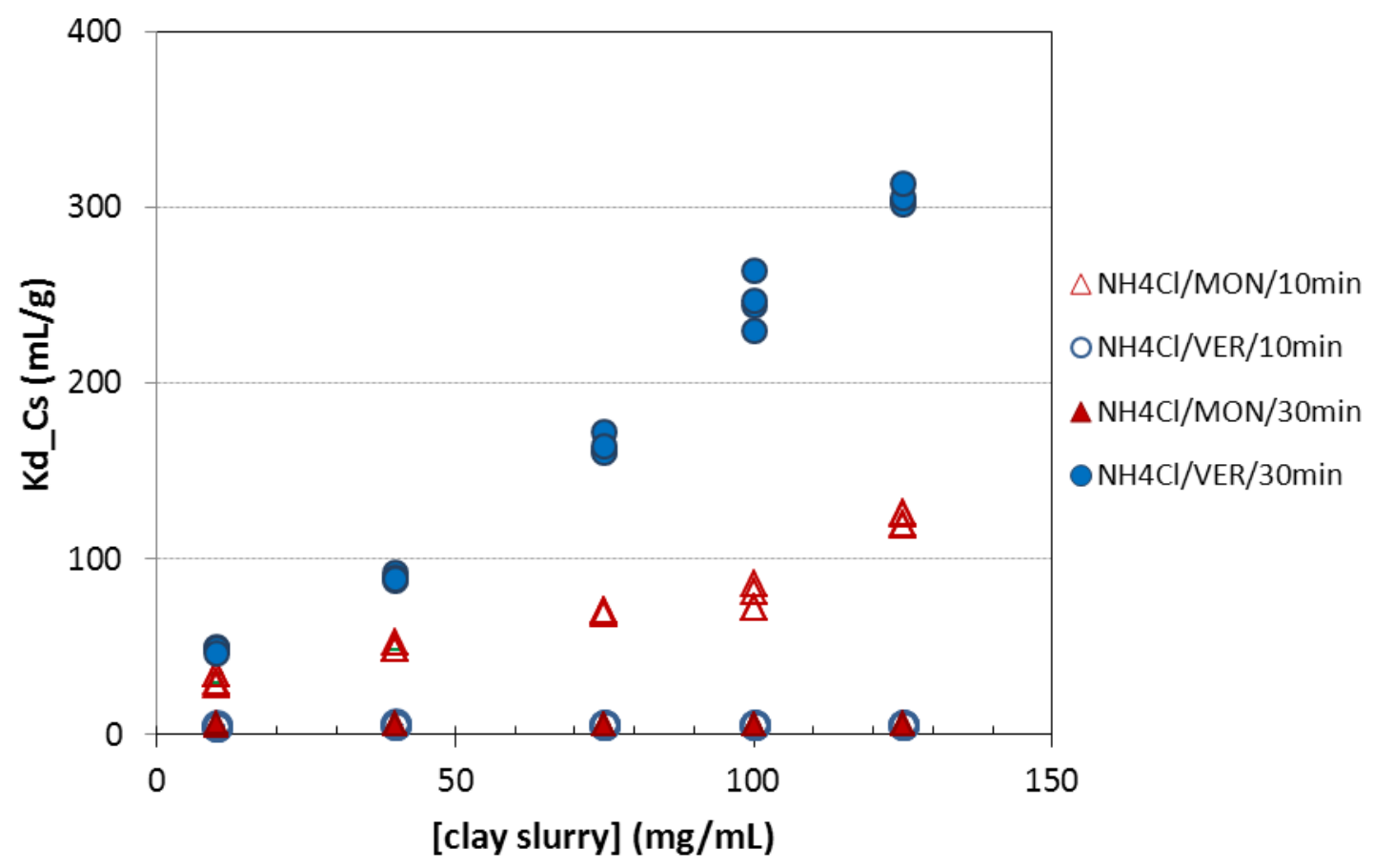

FIGURE 2-12 Sorption of Cs-137 onto clays from solution of $0.5 \mathrm{M} \mathrm{NH}_{4} \mathrm{Cl}$ after $10 \mathrm{~min}$ and $30 \mathrm{~min}$ of gentle agitation. 


\subsubsection{Kinetics of Americium Sorption}

The kinetic tests (Figure 2-13) show that sorption is rapid, reaching equilibrium or peak values within $\sim 10 \mathrm{~min}$. Beyond $60 \mathrm{~min}$, the sorption appears to decrease but still remains high. The sorption increases as

\section{montmorillonite>birnessite> vermiculite>clinoptilolite}

We suspect that appreciable hydrolysis of the $\mathrm{Am}^{3+}$ ion in these solutions promotes the formation of americium hydroxide $\left[\mathrm{Am}(\mathrm{OH})_{\mathrm{n}}{ }^{3-\mathrm{n}}\right]$ species in solution, which may form colloids. Colloids of this type may not follow the reported sorption chemistry for the dissolved species.

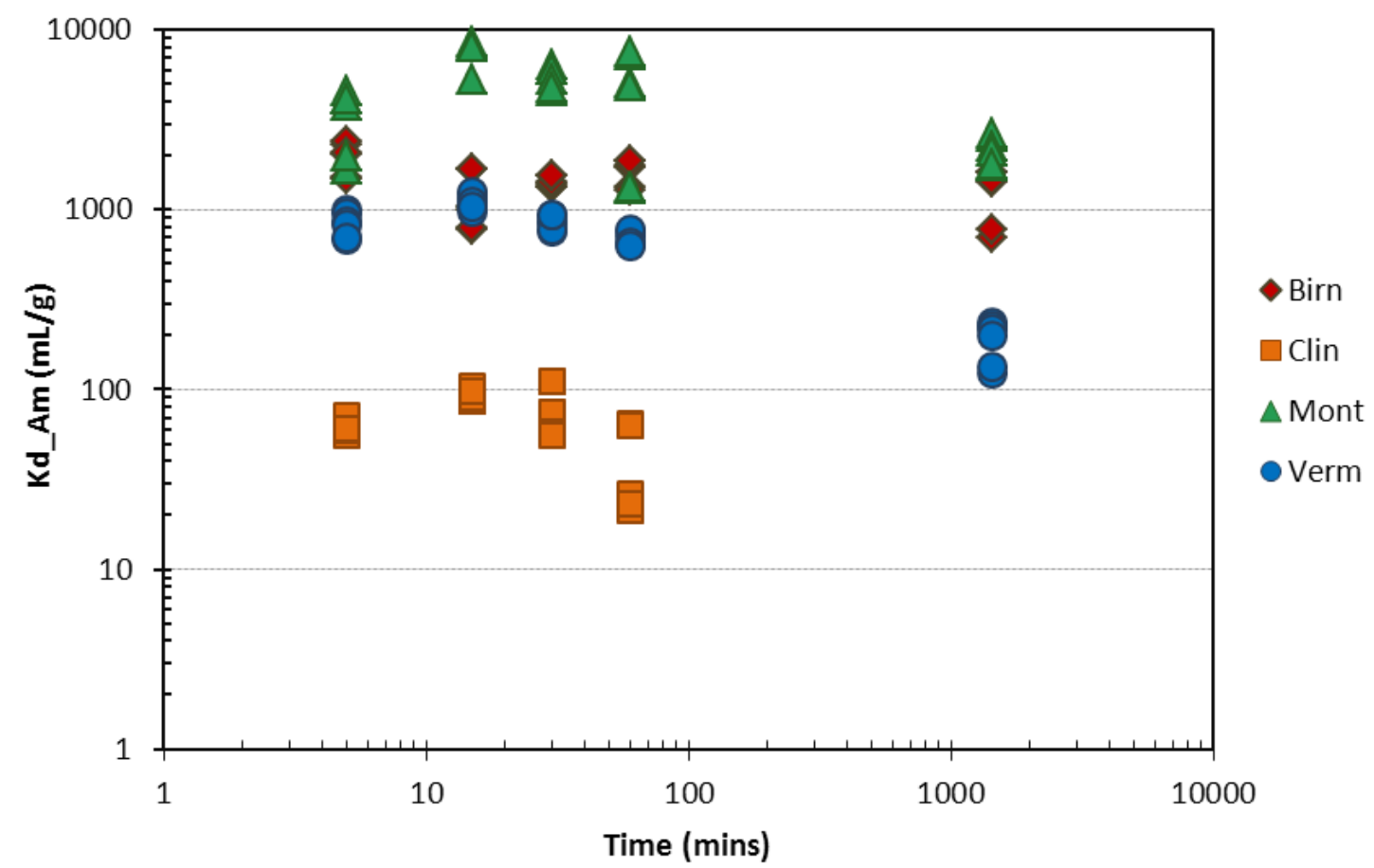

FIGURE 2-13 Kinetics for sorption of Am-241 onto various sorbents from deionized water.

Following the kinetic tests, we completed equilibrium sorption experiments. From $\mathrm{KCl}$ solutions (Figure 2-14) at higher concentration $(>0.01 \mathrm{M})$, the sorption onto clinoptilolite improves with increasing salt. At $0.5 \mathrm{M}$ salt clinoptilolite, birnessite, and vermiculite all sorb americium well, with $\mathrm{K}_{\mathrm{d}}$ 's of $10,000 \mathrm{~mL} / \mathrm{g}$. Sorption onto montmorillonite appears to suffer greatly at high salt content. The improvement in $\mathrm{K}_{\mathrm{d}}$ as salt concentration increases would suggest a salting out effect, although one would expect the potassium to interfere with sorption, as it would have affinity for sorption sites. 


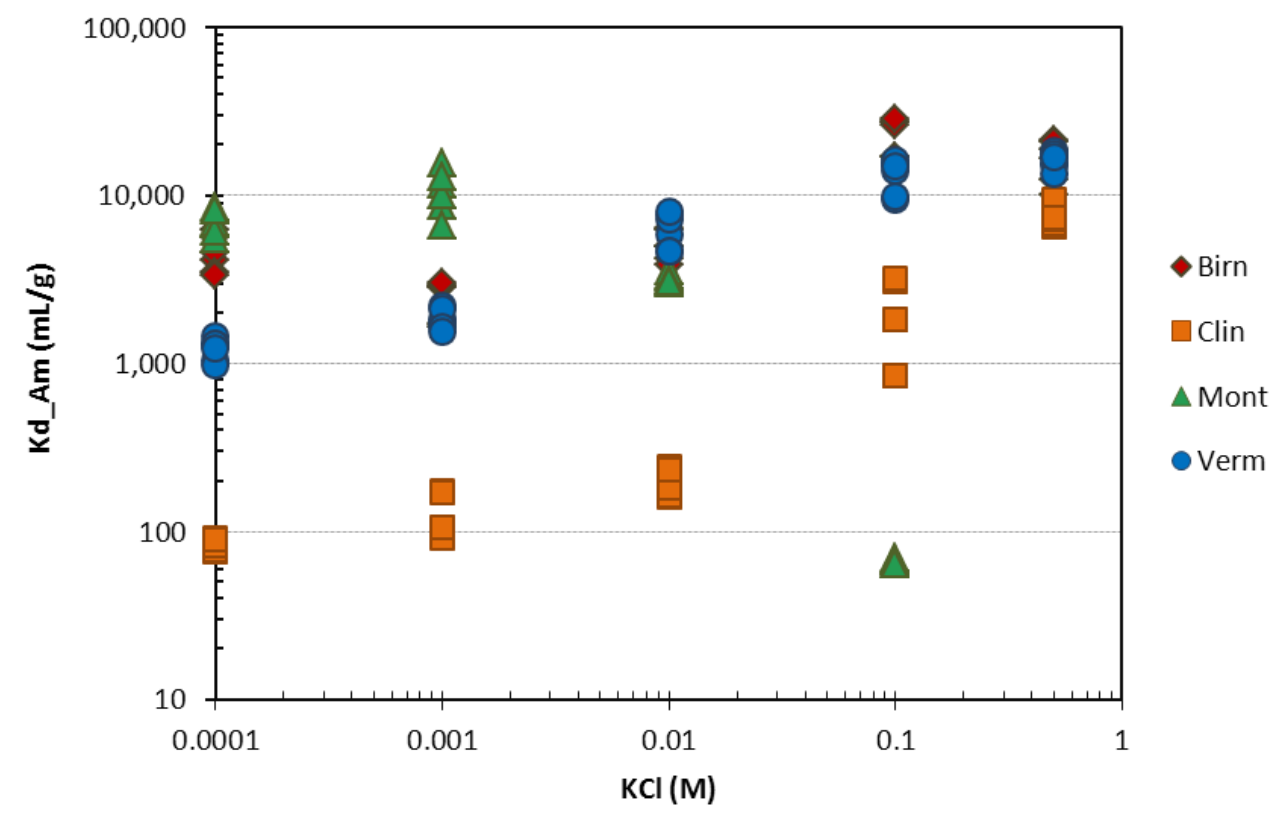

FIGURE 2-14 Sorption of Am-241 onto various sorbents from KCl solution.

From carbonate solution (Figure 2-15), birnessite performs best at high salt content (0.1-0.5 M), with $\mathrm{K}_{\mathrm{d}}$ 's exceeding $100,000 \mathrm{~mL} / \mathrm{g}$ at $0.1 \mathrm{M}$. Sorption values onto montmorillonite and vermiculite are similar and small, while clinoptilolite is the poorest performer. Again, increasing $\mathrm{K}_{\mathrm{d}}$ values as the salt concentration increases would suggest a salting-out effect, with similar lack of interference from the sodium ion as we witnessed for the potassium ion.

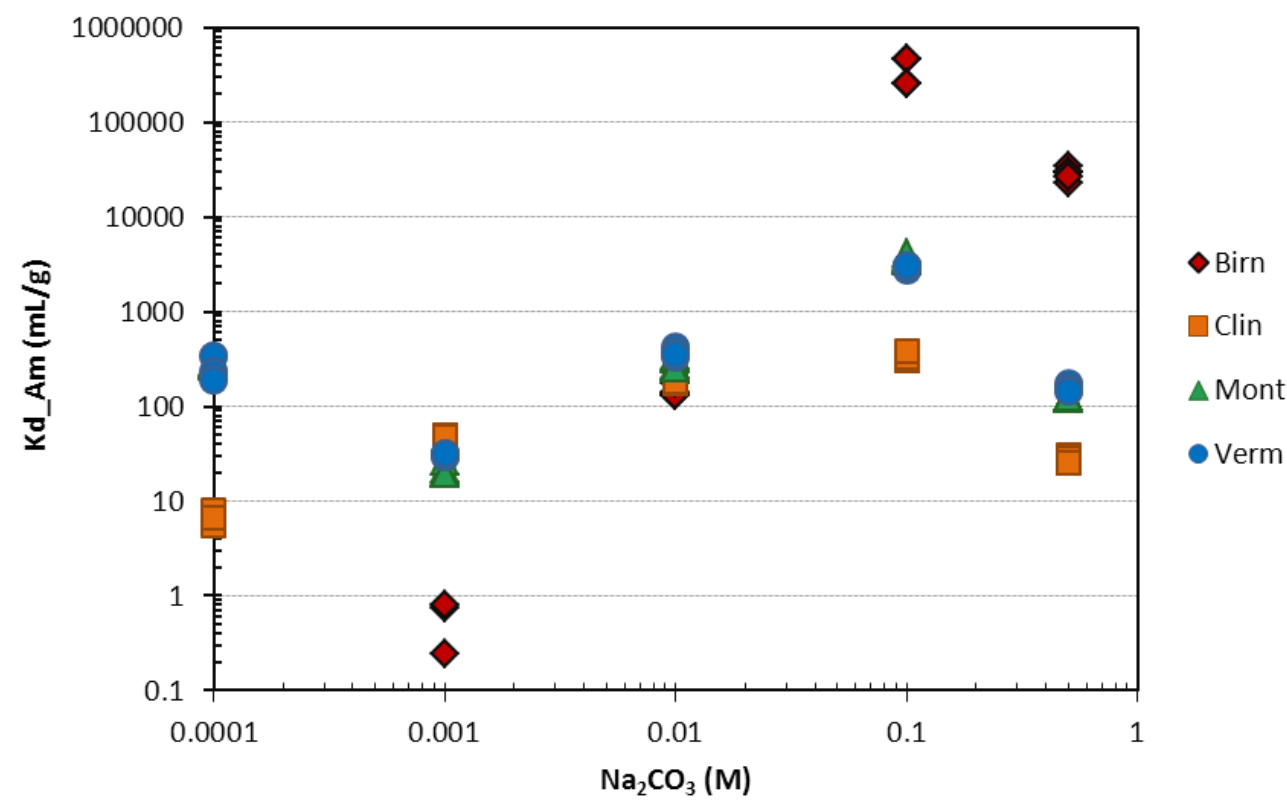

FIGURE 2-15 Sorption of Am-241 onto various sorbents from $\mathrm{Na}_{2} \mathrm{CO}_{3}$ solution. 
From HEDPA solution, the sorption decreases with higher acidity (Figure 2-16). In our region of interest $(0.025 \mathrm{M})$ the extrapolated $\mathrm{K}_{\mathrm{d}}$ 's are $<1000 \mathrm{~mL} / \mathrm{g}$ for birnessite and montmorillonite and $<100 \mathrm{~mL} / \mathrm{g}$ for vermiculite and clinoptilolite. The decrease in $\mathrm{K}_{\mathrm{d}}$ values with increasing acidity is likely due to a combination of the competition of hydrogen for sorption sites as is common among the clays, which prefer sorption at near neutral $\mathrm{pH}$ values, and the chelation effect of the diphosphonic acid anion for the americium cation. The increase in HEDPA would produce a significant concentration of bound americium ions unavailable for sorption onto the clays.

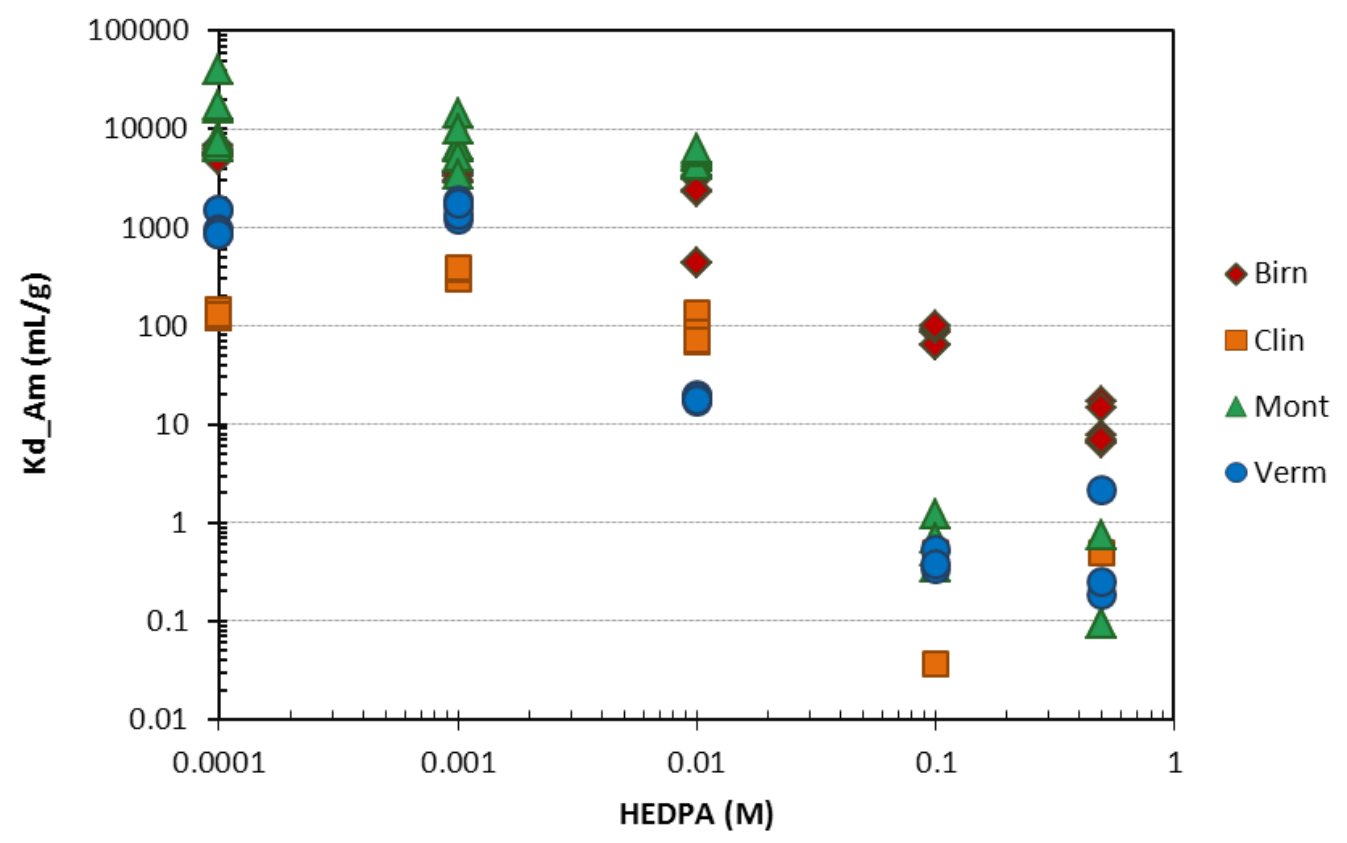

FIGURE 2-16 Sorption of Am-241 onto various sorbents from HEDPA solution. 


\section{MIXED ISOTOPE TESTING (TASK 2)}

\subsection{INTRODUCTION}

The ideal technology would be generic enough to decontaminate a variety of radionuclides without changing the formulation. The SuperGel appears to have that type of generality due to its compatibility with $\mathrm{KCl}, \mathrm{NH}_{4} \mathrm{Cl}, \mathrm{Na}_{2} \mathrm{CO}_{3}$, and $\mathrm{HEDPA}$ reagents. Tests included the decontamination of cesium and americium from concrete, brick, and tile.

\subsection{METHODS}

\subsubsection{Superabsorbent Retention Capacity}

We determined the retention capacity of the gel in solution of 0.025 M HEDPA and $0.25 \mathrm{M} \mathrm{K}_{2} \mathrm{CO}_{3}$ with and without a sequestering agent (Table 3-1). We used Ahlstrom fabric sealed with a Polystar 242 heat sealer. One end was folded, and the other two were sealed to form a pouch. After filling the bag with the polymer and sequestering agent, the end was sealed. Empty bags were weighed to determine the blank correction mass. The bags were soaked for $1 \mathrm{~h}$ in excess solution ( $\sim 50 \mathrm{~g}$ for each test), and then the swollen bags were removed and blotted dry on a lint-free wipe before weighing. The gel polymer mix was 99:1 cross-linked to linear copolymer (cross-linked potassium acrylate-acrylamide copolymer and linear sodium acrylateacrylamide copolymer). Tests with sequestering agent contained $10 \%$ sequestering agent relative to dry mass of polymer.

We calculated the superabsorbent retention capacity (SRC), which is given as (grams of absorbed fluid / grams of dry polymer):

$$
(\mathrm{W} 3-\mathrm{W} 2-\mathrm{W} 1) \div \mathrm{W} 1
$$

where W3 = weight of wet polymer and tea bag; W2 = weight of wet blank tea bag; and $\mathrm{W} 1$ = weight of dry polymer mixture. 
TABLE 3-1 Polymer hydration capacity for mixed isotope solutions ("powder" refers to polymer plus clay contents. HEDPA=etidronic acid or hydroxyethane diphosphonic acid, "Mont" = montmorillonite, "Verm" = vermiculite).

\begin{tabular}{|c|c|c|c|c|c|c|c|}
\hline \multirow[t]{2}{*}{ Sample ID } & \multirow[t]{2}{*}{$\begin{array}{c}\text { Powder } \\
+ \text { T-bag } \\
(\mathrm{g})\end{array}$} & \multirow[t]{2}{*}{$\begin{array}{c}\text { T-bag } \\
(\mathrm{g})\end{array}$} & \multirow[t]{2}{*}{$\begin{array}{c}\text { Hydrated } \\
\text { Contents } \\
(\mathrm{g})\end{array}$} & \multicolumn{3}{|c|}{$\begin{array}{l}\text { Hydration Capacity Solution per } \\
\text { Dry Polymer }(\mathrm{g} / \mathrm{g})\end{array}$} & \multirow[t]{2}{*}{ Solution } \\
\hline & & & & Blank C & rrection & 0.6293 & \\
\hline $2517-42-1$ & 0.160 & 0.160 & 0.8450 & & & & None \\
\hline $2517-42-2$ & 0.160 & 0.160 & 0.7450 & & & & None \\
\hline $2517-42-3$ & 0.162 & 0.162 & 0.7800 & & Average & STD DEV & None \\
\hline $2517-42-4$ & 1.158 & 0.161 & 23.5840 & 21.8623 & 21.8 & 0.3 & $0.025 \mathrm{M}$ HEDPA/0.25 $\mathrm{M} \mathrm{K}_{2} \mathrm{CO}_{3}$ \\
\hline $2517-42-5$ & 1.158 & 0.160 & 23.2760 & 21.5317 & & & $0.025 \mathrm{M}$ HEDPA/0.25 $\mathrm{M} \mathrm{K}_{2} \mathrm{CO}_{3}$ \\
\hline $2517-42-6$ & 1.154 & 0.161 & 23.7350 & 22.1064 & & & $0.025 \mathrm{M}$ HEDPA/0.25 $\mathrm{M} \mathrm{K}_{2} \mathrm{CO}_{3}$ \\
\hline $2517-42-7$ & 1.241 & 0.162 & 24.4480 & 20.9246 & 19.5 & 1.2 & 0.025 M HEDPA/0.25 $\mathrm{M} \mathrm{K}_{2} \mathrm{CO}_{3} / 1 \%$ Mont \\
\hline $2517-42-8$ & 1.253 & 0.162 & 22.6490 & 19.0345 & & & 0.025 M HEDPA/0.25 $\mathrm{M} \mathrm{K}_{2} \mathrm{CO}_{3} / 1 \%$ Mont \\
\hline $2517-42-9$ & 1.253 & 0.160 & 22.2390 & 18.6246 & & & 0.025 M HEDPA/0.25 $\mathrm{M} \mathrm{K}_{2} \mathrm{CO}_{3} / 1 \%$ Mont \\
\hline $2517-42-10$ & 1.255 & 0.161 & 14.6987 & 11.7133 & 12.6 & 0.9 & $0.025 \mathrm{M}$ HEDPA/0.25 $\mathrm{M} \mathrm{K}_{2} \mathrm{CO}_{3} / 1 \%$ Verm \\
\hline $2517-42-11$ & 1.252 & 0.160 & 15.6987 & 12.6533 & & & 0.025 M HEDPA/0.25 $\mathrm{M} \mathrm{K}_{2} \mathrm{CO}_{3} / 1 \%$ Verm \\
\hline $2517-42-12$ & 1.256 & 0.162 & 16.6987 & 13.5406 & & & $0.025 \mathrm{M}$ HEDPA/0.25 $\mathrm{M} \mathrm{K}_{2} \mathrm{CO}_{3} / 1 \%$ Verm \\
\hline $2517-42-13$ & 1.254 & 0.160 & 17.6987 & 14.4565 & 15.4 & 0.9 & 0.025 M HEDPA/0.25 $\mathrm{M} \mathrm{K}_{2} \mathrm{CO}_{3} / 0.5 \% \mathrm{Mon} / 0.5 \%$ Verm \\
\hline $2517-42-14$ & 1.256 & 0.160 & 18.6987 & 15.3407 & & & $0.025 \mathrm{M}$ HEDPA/0.25 $\mathrm{M} \mathrm{K}_{2} \mathrm{CO}_{3} / 0.5 \% \mathrm{Mon} / 0.5 \%$ Verm \\
\hline $2517-42-15$ & 1.254 & 0.161 & 19.6987 & 16.2995 & & & 0.025 M HEDPA/0.25 $\mathrm{M} \mathrm{K}_{2} \mathrm{CO}_{3} / 0.5 \% \mathrm{Mon} / 0.5 \%$ Verm \\
\hline
\end{tabular}




\subsubsection{Concrete Coupon Testing from Mixed Isotope Gel Formulation}

Concrete coupons were prepared for decontamination experiments. All sides except the face were sealed with 5-minute epoxy (Devcon 5-min epoxy 14270). After preparing the stock

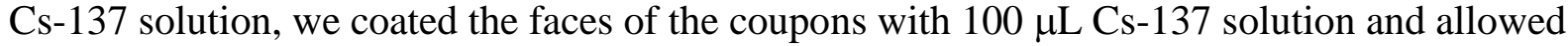
them to dry before wrapping the coupons with plastic wrap for counting. The plastic wrap was polyvinylidene chloride with low permeability to gases and vapors. The coupons were wrapped under ambient conditions (temperature $=22^{\circ} \mathrm{C}$ and $\mathrm{RH}=40 \%$ ). Coupons were counted on a high-purity germanium detector (Detector 1, EG\&G Ortec HPGe, position $10 \mathrm{~cm}, 300$ second live time). Samples were aged for $24 \mathrm{~h}$ in the lab at room temperature. Eight mixed-isotope gel formulations were prepared at 100\% hydration for a 99:1 cross-linked: linear PAM/30\% PAA gel formulation (see results in Section 3.3). Approximately $3 \mathrm{~g}$ gel was applied to the contaminated coupon face and left in contact for $60 \mathrm{~min}$. The gel was removed from the coupon, allowed to dry before wrapping, then analyzed by gamma spectrometry (under same conditions as contamination). All tests were performed in triplicate with background controls.

\subsubsection{Cesium and Americium Decontamination Using Mixed-Isotope Gel Formulation}

We tested the decontamination of cesium and americium by using a 99:1 cross-linked: linear PAM/30\% PAA gel prepared at 100\% hydration employing 0.025 M HEDPA and $0.5 \mathrm{M} \mathrm{K}_{2} \mathrm{CO}_{3}$. Tests were run on six replicates for each urban material: coupons of concrete, brick, and tile. All sides of the coupons except the face were sealed with 5-min epoxy (Devcon 5-min epoxy \#14270) for the decontamination experiments. The faces of the coupons were

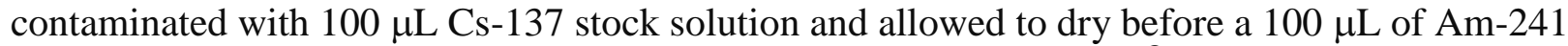
stock solution was added. Coupons were placed into individual Ziploc ${ }^{\circledR}$ plastic bags for counting on a high-purity germanium detector (Detector 1, EG\&G Ortec HPGe, position $20 \mathrm{~cm}, 600$-sec live time). Samples were aged for a week at $40^{\circ} \mathrm{C}$ (however, the humidity control was lost due to a malfunction of the chamber during the aging process, and the relative humidity of the coupons during the aging process is thus unknown). After one week, approximately $3 \mathrm{~g}$ gel was applied to the contaminated coupon face, returned to the humidity chamber at $40^{\circ} \mathrm{C}$ (with open water containers), and left in contact for $60 \mathrm{~min}$. The gel was removed from the coupon, allowed to dry before wrapping, then analyzed by gamma spectrometry (under same counting conditions as the contamination coupons). All tests were run with background controls. 


\subsection{RESULTS}

\subsubsection{Retention Capacity}

Tea bag tests were completed on gel reconstituted in 0.025 M HEDPA and $0.25 \mathrm{M} \mathrm{K}_{2} \mathrm{CO}_{3}$ with and without sequestering agent (Table 3-1). To compare, the tea bag hydration capacities for $1 \mathrm{M} \mathrm{KCl}, 1 \mathrm{M} \mathrm{NH}_{4} \mathrm{Cl}$, and 0.025 $\mathrm{M} \mathrm{HEDPA} / 0.25 \mathrm{M} \mathrm{Na}_{2} \mathrm{CO}_{3}$ are 19.5, 19.8 , and $24.2 \mathrm{~g} / \mathrm{g}$, respectively, in the SuperGel formulation. Vermiculite lowers the hydration capacity to $13-15 \mathrm{~g} / \mathrm{g}$, but the montmorillonite has only a minor effect compared to $\mathrm{HEDPA} / \mathrm{K}_{2} \mathrm{CO}_{3}($ capacity $=21.9 \mathrm{~g} / \mathrm{g})$.

\subsubsection{Decontamination of Cesium from Concrete Using Mixed Isotope Hydrogel Formulations}

Concrete coupons were prepared for decontamination experiments. Eight mixed-isotope gel formulations were prepared at 100\% hydration for a 99:1 cross-linked: linear PAM/30\% PAA gel formulation (Table 3-2).

TABLE 3-2 Gel formulations of 99:1 cross-linked: linear PAM/30\% PAA prepared at $100 \%$ gel hydration using different wash solutions.

\begin{tabular}{ccccccccc}
\hline & \multicolumn{7}{c}{ Gel Wash Solution Formulation } \\
\cline { 2 - 8 } Gel ID & $\mathrm{NH}_{4}^{+}$ & $\mathrm{K}^{+}$ & $\mathrm{Na}^{+}$ & $\mathrm{CO}_{3}^{2-}$ & $\mathrm{Cl}^{-}$ & HEDPA & Mont. & Verm. \\
\hline & & & & & $1 \mathrm{M}$ & & & \\
$2517-40-1$ & $1 \mathrm{M}$ & & & & $1 \mathrm{M}$ & & & \\
$2517-40-2$ & & $1 \mathrm{M}$ & & & $1 \mathrm{M}$ & & \\
$2517-40-3$ & & & $0.5 \mathrm{M}$ & $0.25 \mathrm{M}$ & & $0.025 \mathrm{M}$ & & \\
$2517-40-4$ & $1 \mathrm{M}$ & & & $0.5 \mathrm{M}$ & $0.025 \mathrm{M}$ & & \\
$2517-40-5$ & & $1 \mathrm{M}$ & & $0.5 \mathrm{M}$ & $0.025 \mathrm{M}$ & & \\
$2517-40-6$ & & $1 \mathrm{M}$ & & $0.5 \mathrm{M}$ & $0.025 \mathrm{M}$ & $1 \%$ & \\
$2517-40-7$ & & $1 \mathrm{M}$ & & $0.5 \mathrm{M}$ & $0.025 \mathrm{M}$ & & $1 \%$ \\
$2517-40-8$ & & $1 \mathrm{M}$ & & $0.5 \mathrm{M}$ & $0.025 \mathrm{M}$ & $0.5 \%$ & $0.5 \%$ \\
\hline
\end{tabular}

Results of cesium removal from the concrete coupon decontaminations with the various gel formulations are shown in Figure 3-1. Average decontamination values and triplicate coupon results are represented in the graph, with values for all the tests ranging between 54 and $88 \%$. These cesium-contaminated coupons were aged for 24 hours under ambient conditions $\left(22^{\circ} \mathrm{C}\right.$, $40 \% \mathrm{RH})$. The lowest removal of Cs from the concrete was observed with gel \#3 containing $0.5 \mathrm{M} \mathrm{Na}^{+}, 0.25 \mathrm{M} \mathrm{CO}_{3}{ }^{2-}$, and 0.025 $\mathrm{M} \mathrm{HEDPA}$ (average Cs removal of $63.7 \pm 17.2 \%$ ), and the highest removal was by gel \#2 containing $1 \mathrm{M} \mathrm{K}^{+}$and $1 \mathrm{M} \mathrm{Cl}^{-}$(average Cs removal of $83.3+2.5 \%$ ). The coupon test results using an unpaired t-test for the results from the gel tests were compared for the lowest and highest decontamination. Statistically, there was no difference 
between the test results from gel \#3 and \#2 (Two-sample t-test, T-value $=1.96, p=0.122$ ). Also, the results for the $1 \mathrm{M} \mathrm{KCl}$ prepared gel (\#2) were consistent with earlier reported results for the same gel under similar contaminant aging conditions $(83.3 \pm 2.5 \%$ from Figure $3-1$ and $79.0+9.2 \%$ from 2346-7 and 2346-22 testing).

\subsubsection{Cesium and Americium Decontamination Using Mixed Isotope Gel Formulation}

A mixed-isotope SuperGel was formulated to contain potassium ions for cesium desorption and carbonate and diphosphonic acid for americium dissolution and chelation. Concentrations of the ions and the acid in the gel were combined and formulated to allow optimal substrate decontamination while remaining compatible with the gel. Contaminated coupons were aged for one week at $40^{\circ} \mathrm{C}$ in the humidity chamber (a malfunction of the humidity chamber occurred during testing, and relative humidity conditions are thus unknown).

Figure 3-2 shows results of americium and cesium removal from the concrete coupon decontaminations with the 99:1 cross-linked: linear PAM/30\% PAA gel prepared at 100\% hydration using $0.025 \mathrm{M}$ HEDPA and $0.5 \mathrm{M} \mathrm{K}_{2} \mathrm{CO}_{3}$ gel formulation. Average decontamination values and data from the six coupon replicate tests are represented in the graph. Results for removal of Am-241 from the urban substrate materials in Figure 3-2 follow the trend: tile >> concrete > brick with an average removal of $86 \%, 31 \%$, and $9.8 \%$, respectively. The standard deviation for the replicate tests was low: 7.2, 6.3, and 5.9\% for the concrete, brick, and tile coupon tests, respectively.

Results for removal of Cs-137 from the urban substrate materials in Figure 3-2 follow the trend: tile $>$ concrete $>$ > brick with an average removal of $73 \%, 45 \%$, and $9.8 \%$, respectively. The standard deviation for the replicate tests was also low: $3.4 \%, 4.0 \%$, and $4.7 \%$ for the concrete, brick, and tile coupon tests, respectively. The decontamination results for cesium from tile and concrete coupons are much lower than expected based upon previously reported results. Although the gel formulations used for the comparison are different, the potassium-ion concentration and the contaminant coupon aging conditions are the same $\left(\mathrm{K}^{+}\right.$is assumed to replace $\mathrm{Cs}^{+}$from the substrate). Previously reported results for Cs removal using a $100 \%$ hydrated gel prepared with $1 \mathrm{M} \mathrm{KCl}$ from 7-day aged tile and concrete coupons were $91 \pm 1 \%$ and $59 \pm 4 \%$, respectively. These lower decontaminations shown in Figure 3-2 for cesium removal from concrete and tile are attributed to differences in aging conditions of the coupons, whereby humidity conditions were not maintained at $90 \%$ due to the humidity chamber failure. The $40^{\circ} \mathrm{C}$ temperature was maintained throughout the contaminant aging process for the testing in Figure 2-2 and may have accelerated the bonding of the contaminant to the substrate material and removed pore water, making the decontamination difficult for a one-hour processing time with the $100 \%$ hydrated gel. 


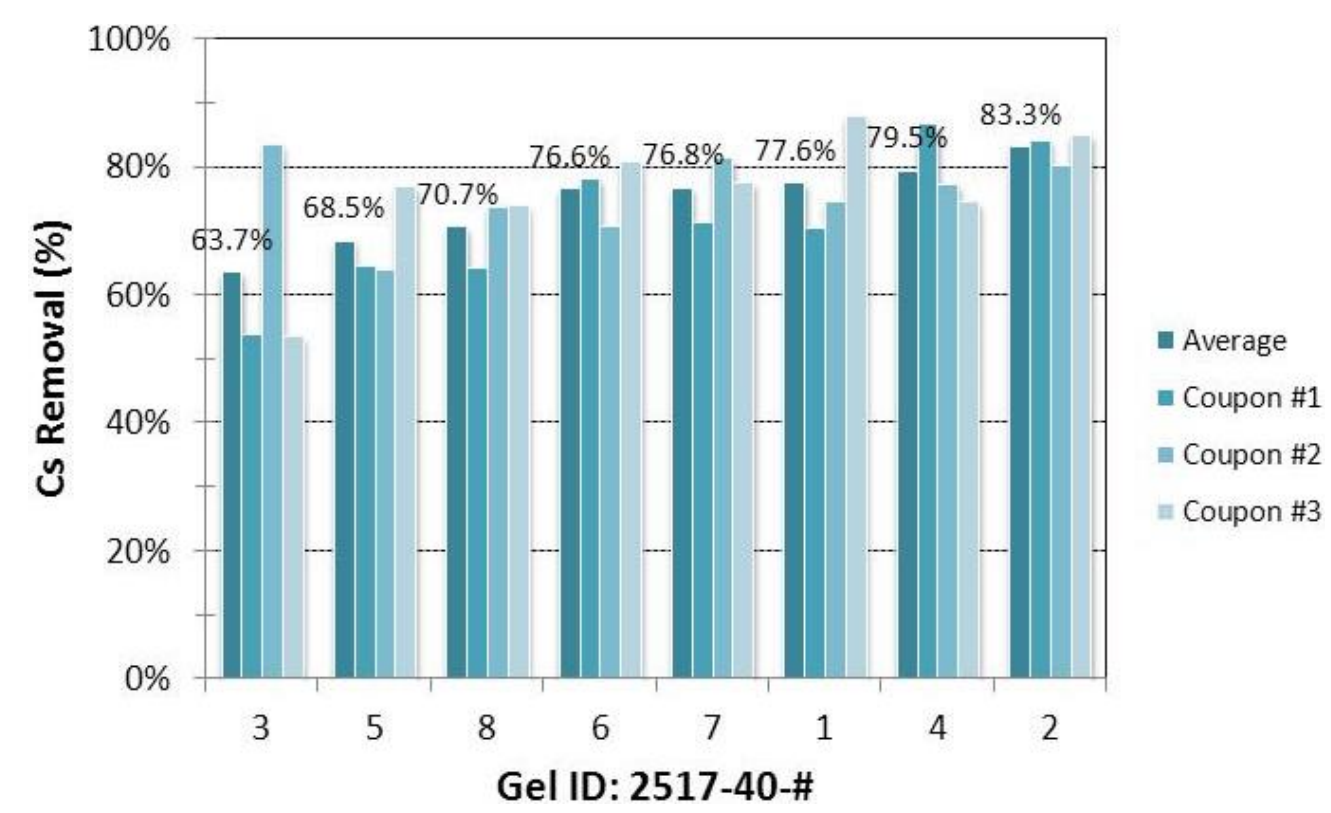

FIGURE 3-1 Decontamination of Cs-137 from concrete coupons using $100 \%$ hydrated gel formulations (99:1 cross-linked: linear PAM/30\% PAA) prepared from different wash solutions. Average cesium removal values are shown in increasing removal efficiency for each gel, and triplicate coupon results are represented to the right of each average. Coupons were aged with its contamination for $24 \mathrm{~h}$ at $22^{\circ} \mathrm{C}$ and $40 \% \mathrm{RH}$.

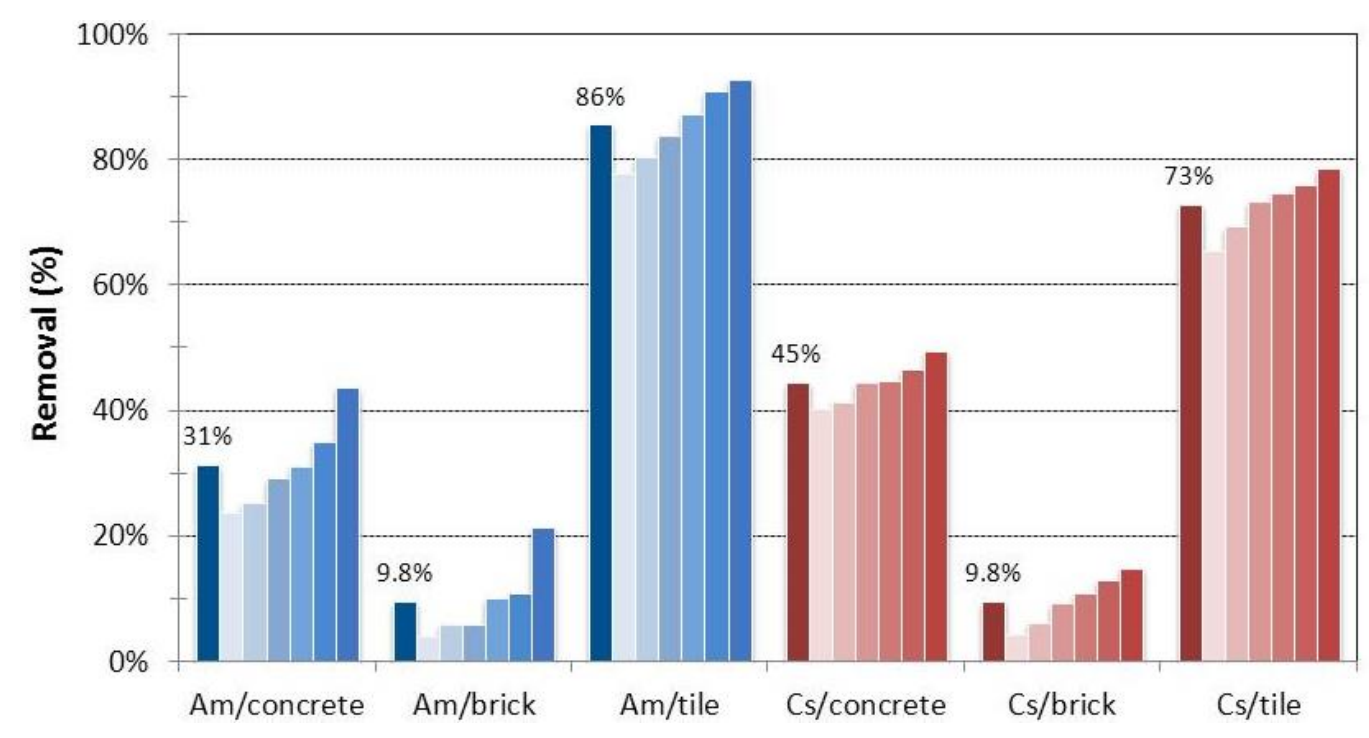

FIGURE 3-2 Decontamination of Am-241 (on left in blue) and Cs-137 (on right in red) from concrete, brick, and tile coupons using $100 \%$ hydrated gel formulation (99:1 cross-linked: linear PAM/30\% PAA in 0.025 M HEDPA and 0.5 $\mathrm{M} \mathrm{K}_{2} \mathrm{CO}_{3}$ ). Average cesium removal values are shown as data labels in the figure. Individual coupon replicate results are shown to the right of each average in increasing removal efficiency for each substrate. Coupons with its contamination were aged for one week at $40^{\circ} \mathrm{C}$ (relative humidity was not available). 


\section{EFFECT OF VARIATION IN CONCENTRATION OF AMERICIUM AND CESIUM CONTAMINATION (TASK 3)}

\subsection{INTRODUCTION}

The tests in Section 3 were performed at a single concentration of cesium and americium contamination. To be thorough, we proposed testing of cesium and americium within a range of concentrations consistent with three zones of contamination defined by the Liberty Rad Ex exercise (Figure 4-1). Coupons were prepared spanning the specified contamination range for cesium and americium. Concrete, tile, and brick samples were tested.

\subsection{METHODS}

Coupon decontamination for cesium covered the estimated maximum surface activity for streets and exterior building walls in the decontamination zones following a radiological dispersal device incident. While the zone definitions and mitigation activities were changed from previous reporting [5] and updated with assumptions from the National Tier 2 Full-Scale Radiological Dispersion Device Exercise (also known as Liberty RadEx) sponsored by the U.S. Environmental Protection Agency (EPA), [6] our coupon contaminant surface activities cover the decontamination zones used for current remediation efforts $\left(0.24\right.$ and $0.112 \mathrm{mCi} / \mathrm{m}^{2}$ for Zones 2 and 3, respectively). Decontamination strategies vary with deposited radioactivity. For example, in Zone 1, the plan is for demolition of $90 \%$ of contaminated structures and decontamination of the remaining 10\%. For Zones 2 and 3, the reverse is expected, $10 \%$ demolition and $90 \%$ decontamination. The plume profile model (Figure 4-1) shows the expected zones and radiation dose limits within each for release of Cs-137 in the exercise.

For our tests, concrete coupons were contaminated with Cs-137 activity levels spanning two orders of magnitude and covering the maximum and minimum activities for the decontamination zones (Figure 4-2). Stable cesium was added to trace Cs-137 spiked solutions to achieve high contamination levels while minimizing testing dose levels. As stable isotopes are unavailable for americium and the disposition and chemistry of lanthanide analogues would need verification, only tracer tests with Am-241 were performed at a concentration corresponding to Zone 3 levels. 


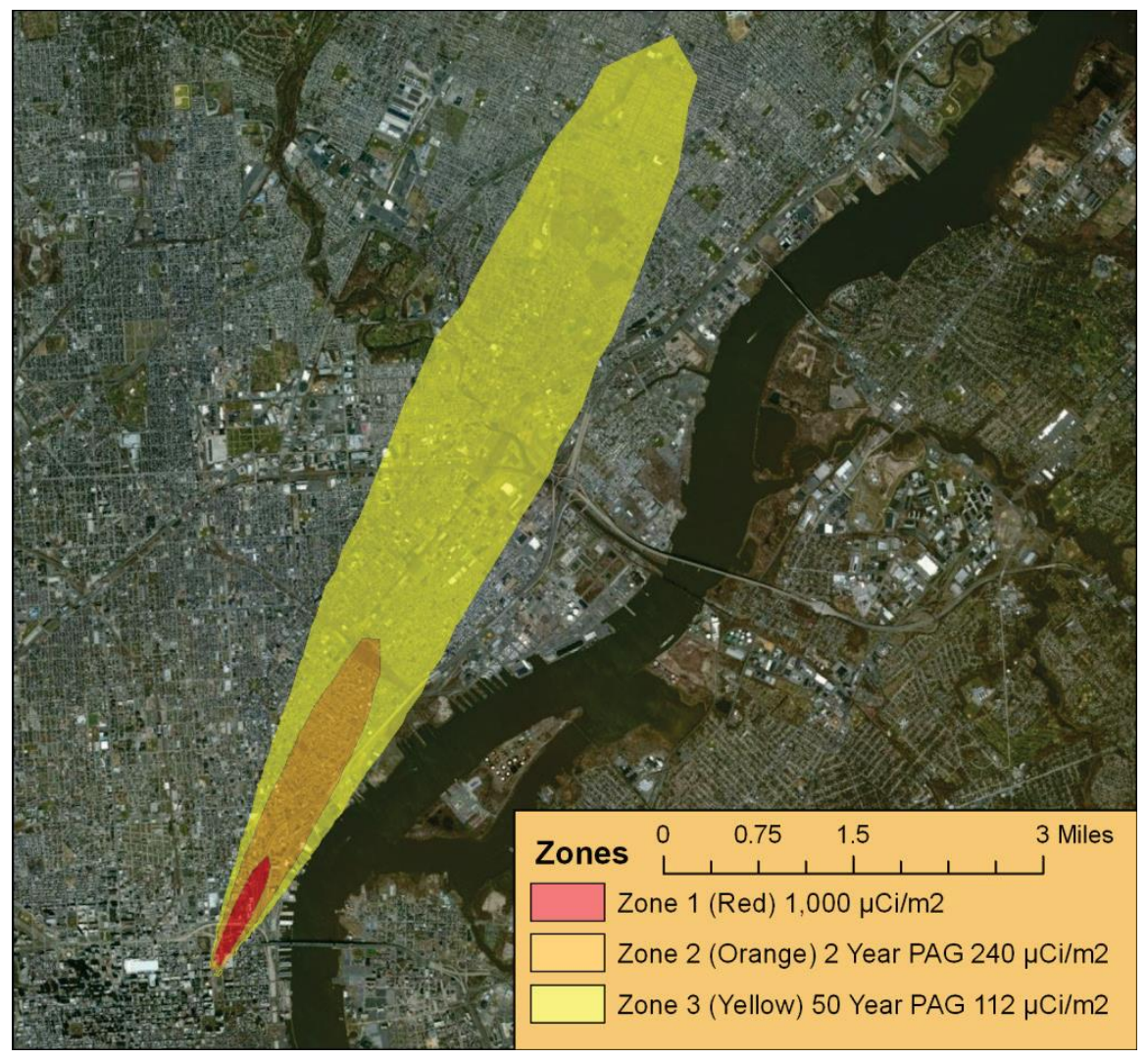

FIGURE 4-1 Plume profile used in Liberty RadEx for a hypothetical RDD release. [6,7]

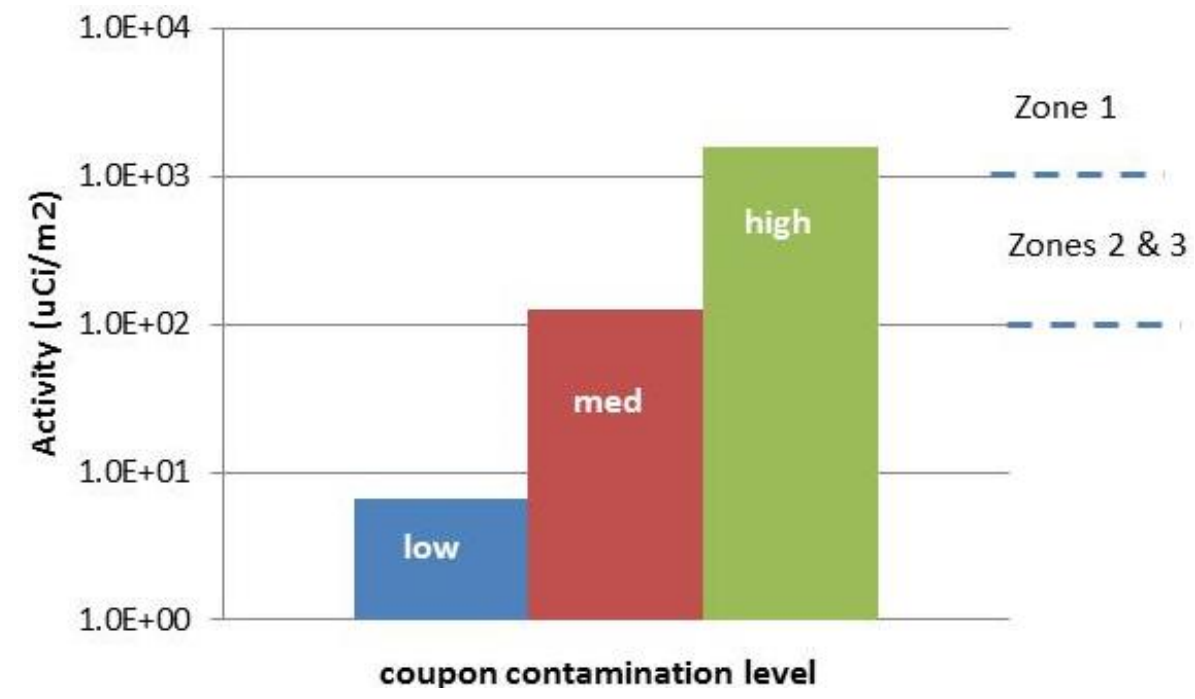

FIGURE 4-2 Activity levels for coupons contaminated with Cs-137 and cold cesium carrier. 
Using four different wash solutions, we tested the decontamination of cesium and americium with a 99:1 cross-linked: linear PAM/30\% PAA gel prepared at 100\% hydration. Tests were run in triplicate, with each gel formulation using the concrete material. All sides of the coupons except the face were sealed with 5-minute epoxy (Devcon 5-min epoxy \#14270) for the decontamination experiments. The faces of the coupons were contaminated with $100 \mu \mathrm{L} \mathrm{Cs-}$ 137 spiked stock solutions (prepared at low, medium, and high concentration with nonradioactive cesium as appropriate) or $100 \mu \mathrm{L}$ of Am-241 stock solution (only one concentration, since non-radioactive Am is unavailable). Coupons were allowed to dry and then bagged into individual Ziploc ${ }^{\circledR}$ plastic bags for counting on a high-purity germanium detector (Detector 1, EG\&G Ortec HPGe, position $20 \mathrm{~cm}, 600$-sec live time). Samples were aged with its contamination for seven days at room temperature. After one week, approximately 3-g gel was applied to the contaminated coupon face and left in contact with the contaminated coupon surface for $60 \mathrm{~min}$. The gel was removed from the coupon, allowed to dry before wrapping, then analyzed by gamma spectrometry (under same counting conditions as the contamination coupons).

\subsection{RESULTS}

Results of cesium and americium removal from the concrete coupon decontaminations with the 99:1 cross-linked: linear PAM/30\% PAA gel prepared at 100\% hydration using $0.025 \mathrm{M}$ HEDPA and $0.5 \mathrm{M} \mathrm{K}_{2} \mathrm{CO}_{3}$ gel formulation are shown in Tables 4-1 and 4-2, respectively. The results for cesium decontamination appear consistent with our previous testing that shows $\sim 70 \%$ removal of cesium in a single decontamination application.

TABLE 4-1 Cesium (Cs) removal at low, medium, and high contaminant concentrations from concrete using 100\% hydrated gel formulations (99:1 crosslinked: linear PAM/30\% PAA with various wash solutions). Individual coupon replicate results are shown with average and standard deviations for removal efficiency for each gel formulation.

\begin{tabular}{lccccc}
\hline Coupon ID & Gel Wash Solution & $\begin{array}{c}\text { Mass } \\
\text { of Gel } \\
(\mathrm{g})\end{array}$ & $\begin{array}{c}\text { Cs } \\
\text { Removal } \\
(\%)\end{array}$ & $\begin{array}{c}\text { Average } \\
\text { Removal } \\
(\%)\end{array}$ & $\begin{array}{c}\text { Std Dev } \\
\text { Removal } \\
(\%)\end{array}$ \\
\hline low Cs & & & & & \\
$2517-91-\mathrm{LC} 1$ & $1 \mathrm{M} \mathrm{NH}_{4} \mathrm{Cl}$ & 3.1 & 78.6 & 76.1 & 2.2 \\
2517-91-LC2 & & 2.7 & 75.3 & & \\
2517-91-LC3 & & 3.2 & 74.4 & & \\
2517-91-LC4 & $1 \mathrm{M} \mathrm{KCl}$ & 2.4 & 71.6 & 71.3 & 7.2 \\
2517-91-LC5 & & 3.1 & 78.4 & & \\
2517-91-LC6 & & 2.8 & 64.1 & & \\
2517-91-LC7 & $0.5 \mathrm{M} \mathrm{K}_{2} \mathrm{CO}_{3}$ & 3.1 & 77.0 & 78.7 & 8.9 \\
2517-91-LC8 & & 3.4 & 70.8 & & \\
2517-91-LC9 & & 2.6 & 88.4 & & \\
\hline
\end{tabular}


TABLE 4-1 (Cont.)

\begin{tabular}{|c|c|c|c|c|c|}
\hline Coupon ID & Gel Wash Solution & $\begin{array}{c}\text { Mass } \\
\text { of Gel } \\
(\mathrm{g}) \\
\end{array}$ & $\begin{array}{c}\text { Cs } \\
\text { Removal } \\
(\%)\end{array}$ & $\begin{array}{c}\text { Average } \\
\text { Removal } \\
(\%)\end{array}$ & $\begin{array}{c}\text { Std Dev } \\
\text { Removal } \\
(\%)\end{array}$ \\
\hline \multicolumn{6}{|l|}{ low Cs (Cont.) } \\
\hline 2517-91-LC10 & $0.5 \mathrm{M} \mathrm{K}_{2} \mathrm{CO}_{3} / 0.025 \mathrm{M}$ HEDPA & 3.3 & 77.2 & 64.5 & 11.0 \\
\hline 2517-91-LC11 & & 3.3 & 58.7 & & \\
\hline 2517-91-LC12 & & 3.0 & 57.8 & & \\
\hline \multicolumn{6}{|l|}{ medium Cs } \\
\hline 2517-84-MC1 & $1 \mathrm{M} \mathrm{NH}_{4} \mathrm{Cl}$ & 3.2 & 70.3 & 67.7 & 4.4 \\
\hline 2517-84-MC2 & & 2.5 & 62.7 & & \\
\hline 2517-84-MC3 & & 3.0 & 70.2 & & \\
\hline 2517-84-MC4 & $1 \mathrm{M} \mathrm{KCl}$ & 3.2 & 69.2 & 63.6 & 4.9 \\
\hline 2517-84-MC5 & & 2.6 & 60.7 & & \\
\hline 2517-84-MC6 & & 2.3 & 60.8 & & \\
\hline 2517-84-MC7 & $0.5 \mathrm{M} \mathrm{K}_{2} \mathrm{CO}_{3}$ & 3.0 & 79.0 & 76.1 & 7.3 \\
\hline 2517-84-MC8 & & 2.9 & 67.9 & & \\
\hline 2517-84-MC9 & & 2.8 & 81.6 & & \\
\hline 2517-84-MC10 & $0.5 \mathrm{M} \mathrm{K}_{2} \mathrm{CO}_{3} / 0.025 \mathrm{M}$ HEDPA & 3.4 & 47.3 & 60.8 & 12.2 \\
\hline 2517-84-MC11 & & 3.6 & 64.4 & & \\
\hline 2517-84-MC12 & & 3.4 & 70.8 & & \\
\hline \multicolumn{6}{|l|}{ high Cs } \\
\hline 2517-89-HC1 & $1 \mathrm{M} \mathrm{NH}_{4} \mathrm{Cl}$ & 3.2 & 87.5 & 87.0 & 0.51 \\
\hline 2517-89-HC2 & & 2.7 & 86.4 & & \\
\hline 2517-89-HC3 & & 3.6 & 87.0 & & \\
\hline 2517-89-HC4 & $1 \mathrm{M} \mathrm{KCl}$ & 3.1 & 57.6 & 67.4 & 13.9 \\
\hline 2517-89-HC5 & & 4.1 & 61.3 & & \\
\hline 2517-89-HC6 & & 4.0 & 83.3 & & \\
\hline 2517-89-HC7 & $0.5 \mathrm{M} \mathrm{K}_{2} \mathrm{CO}_{3}$ & 3.3 & 67.0 & 66.6 & 13.1 \\
\hline 2517-89-HC8 & & 3.1 & 79.4 & & \\
\hline 2517-89-HC9 & & 3.2 & 53.2 & & \\
\hline 2517-89-HC10 & $0.5 \mathrm{M} \mathrm{K}_{2} \mathrm{CO}_{3} / 0.025 \mathrm{M}$ HEDPA & 3.8 & 75.0 & 61.0 & 12.2 \\
\hline 2517-89-HC11 & & 3.4 & 53.8 & & \\
\hline 2517-89-HC12 & & 4.0 & 54.3 & & \\
\hline
\end{tabular}


TABLE 4-2 Americium (Am) removal from concrete using $100 \%$ hydrated gel formulations (99:1 cross-linked: linear PAM/30\% PAA with various wash solutions). Individual coupon replicate results are shown with average and standard deviations for removal efficiency for each gel formulation.

\begin{tabular}{cccccc}
\hline & & $\begin{array}{c}\text { Mass } \\
\text { of Gel } \\
\text { Coupon ID }\end{array}$ & $\begin{array}{c}\text { Am } \\
\text { Removal Wash Solution } \\
(\%)\end{array}$ & $\begin{array}{c}\text { Average } \\
\text { Removal } \\
(\%)\end{array}$ & $\begin{array}{c}\text { Std Dev } \\
\text { Removal } \\
(\%)\end{array}$ \\
\hline & & & & & \\
$2517-92-\mathrm{Am} 1$ & $0.5 \mathrm{M} \mathrm{K}_{2} \mathrm{CO}_{3}$ & 2.6 & 15.7 & 18.6 & 2.9 \\
$2517-92-\mathrm{Am} 2$ & & 2.2 & 18.5 & & \\
$2517-92-\mathrm{Am} 3$ & & 2.5 & 21.5 & & \multirow{2}{*}{5.8} \\
$2517-92-\mathrm{Am} 4$ & $0.5 \mathrm{M} \mathrm{K}_{2} \mathrm{CO}_{3} / 0.025 \mathrm{M}$ & 3.3 & 66.7 & 73.0 & \\
$2517-92-\mathrm{Am} 5$ & $\mathrm{HEDPA}$ & 3.4 & 78.1 & & \\
$2517-92-\mathrm{Am} 6$ & & 3.1 & 74.3 & & \\
\hline
\end{tabular}

Importantly, the decontamination results for americium are much improved with this combined formulation. We observed $73 \%$ decontamination for the combined SuperGel formulation (appropriate for both Cs and Am decontamination), where before we had only achieved $\sim 50 \%$ decontamination of Am from concrete. We suspect that the increased carbonate concentration used in the present tests, the new counter ion to the potassium cation needed for cesium ion exchange, might explain the improved results.

\section{PILOT-SCALE TESTING (TASK 4)}

\subsection{INTRODUCTION}

To this point, our research has been focused on benchtop experiments with small test surfaces of urban materials. It is vitally important that we complete pilot-scale tests to assess the variability of the tests' data with larger sample areas. Pilot-scale tests involved larger test samples (0.02-0.09 $\mathrm{m}^{2}$ or 6-12 in. square coupons) contaminated to levels consistent with previous testing and dissemination of the wash solution and gel using actual methods expected in the field. Testing at the pilot-scale level was performed at Idaho National Laboratory and Argonne National Laboratory. 


\subsection{METHODS}

\subsubsection{Idaho Testing of Combined Formulation}

\subsubsection{Materials}

Concrete coupons were prepared in a single batch of concrete made from Type II Portland cement. The ready-mix company (Burns Brothers Redi-Mix, Idaho Falls, Idaho) provided physical data on the cement clinker used in the concrete mix (tricalcium silicate $=$ $57.6 \%$; dicalcium silicate $=21.1 \%$; tricalcium aluminate $=4.5 \%$; tetracalcium aluminoferrite $=$ 8.7\%; minor constituents $=8.1 \%$ ) and meets the ASTM C1501 requirement for Type I and II Portland cement.

To make the concrete coupons, the wet concrete was poured into 0.9-m square plywood forms (approximately 4-cm thick) with the surface exposed. The surface was then "floated" to get the smaller aggregate and cement paste to the top (the surface used for this evaluation), and then was cured for 21 days. Following curing, the 4-cm thick squares were cut with a laserguided rock saw to the desired concrete coupon size of approximately $15 \mathrm{~cm} \times 15 \mathrm{~cm}$. The coupons had a surface finish that was consistent across all the coupons. All of these coupons were contaminated with 2.5 milliliters $(\mathrm{mL})$ of unbuffered, slightly acidic aqueous solution containing approximately 20 nanocurie (nCi)/mL Am-243 which corresponds to an activity level of approximately $50 \mathrm{nCi}$ per coupon $( \pm 5 \mathrm{nCi})$. This was delivered using a compressed air line connected to a syringe to produce a fine mist. Coupons were contaminated approximately two weeks before use.

\subsubsection{Pilot-scale Demonstration}

The SuperGel (0.5 $\mathrm{M} \mathrm{K}_{2} \mathrm{CO}_{3}, 0.025 \mathrm{M}$ HEDPA, in 99/1 cross linked to linear at $100 \%$ hydration) was applied approximately 6-mm thick to the ten coupons using a 4-in. spatula (Figure 5-1), was allowed to dwell on the surface for 90 minutes, and then was removed with a wet vacuum (Little Green, Bissell, Grand Rapids, Michigan). 


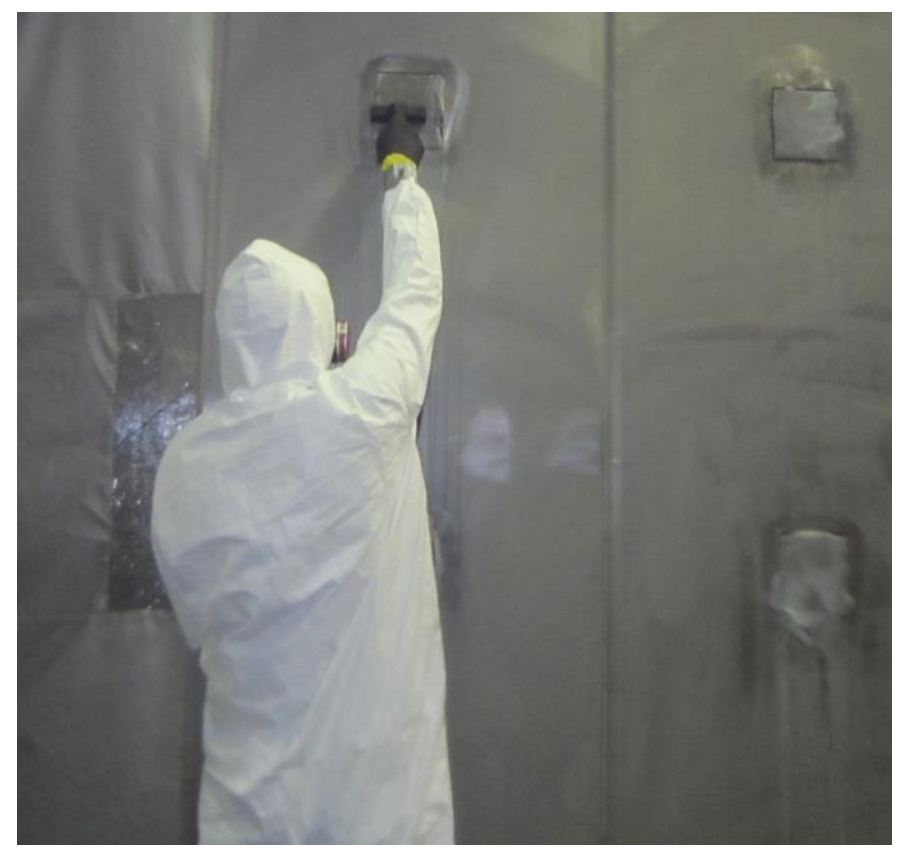

FIGURE 5-1. Test wall containing the nine concrete samples used in the testing at Idaho National Laboratory using the mixed-isotope formulation of the SuperGel.

\subsubsection{Argonne Testing of Combined Formulation}

\subsubsection{Materials}

Argonne received seventeen $6 \times 6 \times 1$ in. coupons from the sponsor. These coupons were left in their original package for six months before testing. Prior to testing, each coupon was vacuumed (Rigid Shop-Vac) to remove the cement dust from the surface before application of epoxy to seal the coupon side surfaces. Details on the preparation of these concrete coupons are unknown but they were intended to be replicas of the small coupons used in the tests described in Sections 3 and 4.

Coupon surfaces were sealed prior to the decontamination testing by epoxying coupon sides (Figure 5-2) using a wooden applicator stick. Epoxy was not applied to the bottom surface of the coupons. The bottom of each coupon was labeled with a unique identifier. 


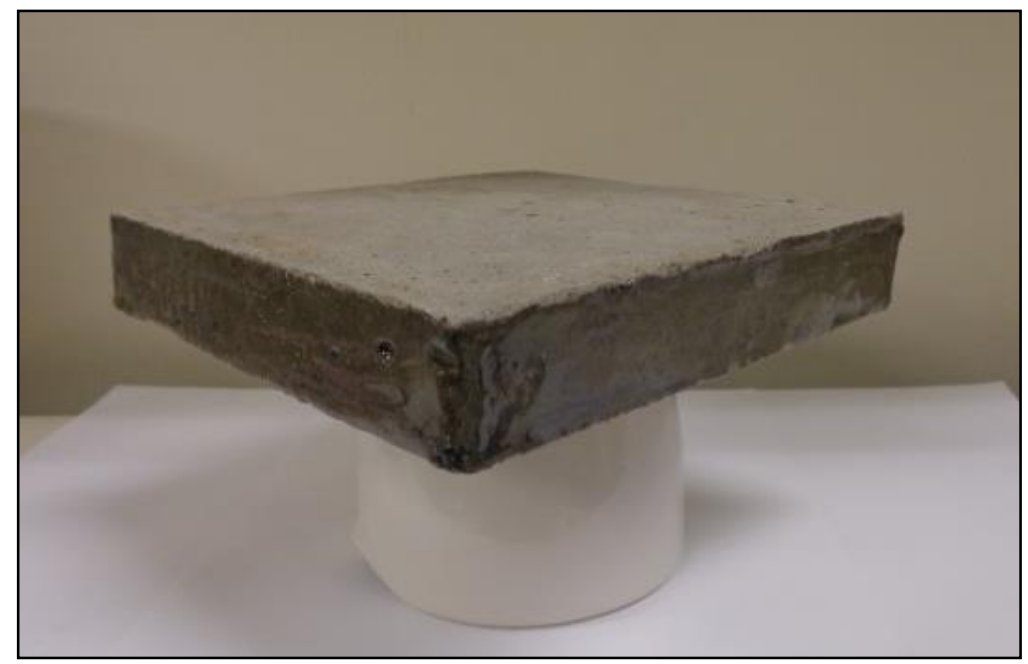

FIGURE 5-2 Photograph of epoxied coupon side surfaces.

Contaminated coupons were prepared from a working stock solution of Cs-137 $(\sim 500,000 \mathrm{cpm} / \mathrm{mL})$. We contaminated each coupon by distributing $4.0 \mathrm{~mL}$ of Cs-137 solution to the face of each coupon in quadrants, yielding $\sim 1.0 \mu \mathrm{C}$ per sample. The Cs-137 solution was soaked up immediately, and the coupon surface appeared to be dry. In a few cases, some areas remained wet with droplets and were allowed to air dry for 20-30 min. We observed no visual differences between these test samples and the others that dried upon contact with the solution. When all coupons were dry, each coupon was double-bagged in a polyethylene (Ziploc ${ }^{\circledR}$ ) bag.

We prepared the gel formulation in $1.0 \mathrm{M} \mathrm{KCl}$ using a 99:1 cross-linked to linear ratio of Hydrosource polymers with $10 \%$ by mass of polymer in CST (IONSIV 911). A $1.0 \mathrm{M} \mathrm{KCl}$ solution was prepared and mixed using an impeller mixer until $\mathrm{KCl}$ pellets were dissolved. Dry materials (cross-linked/linear polymers, CST) were weighed and mixed together and were slowly added to $1.0 \mathrm{M} \mathrm{KCl}$ solution while mixing for $30 \mathrm{~min}$. The gel was prepared at least four days before the demonstrations, covered with plastic wrap, and placed in the refrigerator.

\subsubsection{Gamma Analyses}

Gamma measurements were performed on contaminated, decontaminated, control, and background coupons using Argonne's low-background counting facility. The gamma analyzer was set to count the $667 \mathrm{keV}$ photopeak of Cs-137 against an HPGe detector at a distance of $13.3 \mathrm{~cm}$ from the detector face to the contaminated concrete surface. Coupons were counted for 60 or $180 \mathrm{sec}$. The coupons from pilot tests \#1 and \#2 were counted in triplicate by rotating the coupon $1 / 3$ clockwise between each measurement and centering the coupon about the detector face each time. The counting statistics for the triplicate counts on individual coupons indicated very consistent results, with typically less than 1\% RSD. Thus, coupons for pilot test \#3 were only counted once. 


\subsubsection{Density and Porosity Measurements}

We measured the density and porosity of the concrete used in the demonstration. Using a hammer and chisel, we removed several pieces from the corner of one coupon for measurement using a Micropycnometer (Quantachrome, MPY-5) run with pure helium gas. The true volume of concrete $V_{\text {concrete }}$ (excluding pore volume) was calculated using the following expression:

$$
\mathrm{V}_{\text {concrete }}=\mathrm{V}_{\mathrm{c}}-\mathrm{V}_{\mathrm{R}}\left[\mathrm{P}_{1} / \mathrm{P}_{2}-1\right]
$$

where $P_{1}$ is the gas pressure initially in the system, $P_{2}$ is the gas pressure after release into the cell, $V_{C}$ is the volume of the cell $\left(8.300 \mathrm{~cm}^{3}\right)$, and $V_{R}$ is the reference volume $\left(6.605 \mathrm{~cm}^{3}\right)$.

Calibration spheres (stainless steel, $V_{\text {spheres }}=2.145 \mathrm{~m}^{3}$ ) were run to check the calibration of the unit prior to measuring the concrete density. The following values were measured and were in good agreement:

$$
\begin{aligned}
& \text { Run \#1: } \mathrm{P}_{1}=17.050, \mathrm{P}_{2}=8.824, V_{\text {spheres }}=2.143 \mathrm{~cm}^{3} . \\
& \text { Run \#2: } \mathrm{P}_{1}=17.126, \mathrm{P}_{2}=8.863, V_{\text {spheres }}=2.142 \mathrm{~cm}^{3} .
\end{aligned}
$$

The concrete pieces were placed in the sample cell and weighed $\left(m_{\text {concrete }}\right)$. Two measurements were taken, and the average value and range were reported. The true density $\rho_{\text {true }}$ was computed by $m_{\text {concrete }} / V_{\text {concrete }}$.

To measure the bulk density, Archimedes method was used. Deionized water at room temperature was measured to a volume of $15 \mathrm{~mL}$ into a graduated cylinder. The same concrete pieces used in the pycnometer were wetted with deionized water to ensure removal of air bubbles from the internal porosity. The surface water was removed by quickly blotting onto a lint-free wipe. After dropping the pieces into the graduated cylinder, water was removed and weighed until the water line measured exactly $15 \mathrm{~mL}$. The mass of water was used to calculate the volume of water displaced by the concrete pieces. This value for volume represented the geometric volume of concrete $V_{\text {geom }}$. The bulk density $\rho_{\text {bulk }}$ was determined by $m_{\text {concrete }} / V_{\text {geom }}$ and the pore density as $\rho_{\text {bulk }} / \rho_{\text {true }}$.

\subsubsection{Pilot-scale Demonstrations}

We performed three demonstrations of the decontamination process for removing Cs-137 from large concrete test samples. These tests are outlined in Table 5-1. Details for each of the pilot-scale demonstrations are described in the following text.

In Argonne Pilot Test \#1, the rough backside of nine coupons was contaminated as described in Section 5.2.2.1. Coupons were selected for pilot test \#1 based upon pore surface. Some of the coupons had large deep pores (Figure 5-3) and were not selected for the first demonstration. Technical problems with the humidity chamber resulted in a variation in the aging conditions for the coupons. For three days, the coupons inside the humidity chamber were held at $90 \%$ relative humidity and $40^{\circ} \mathrm{C}$, but over the weekend the humidity may have dropped 
below $90 \%$. When we returned to the lab on Monday morning, the humidity read $70 \%$ at $40^{\circ} \mathrm{C}$. These issues with the humidity chamber forced us to shut down the chamber for the remaining two days. The coupons were left inside the chamber with open water containers placed in the bottom of the chamber; the temperature in the chamber was allowed to return to ambient $\left(22^{\circ} \mathrm{C}\right)$.

TABLE 5-1 Summary of Argonne pilot-test conditions for cesium-137 contaminated concrete coupons.

\begin{tabular}{ccccc}
\hline $\begin{array}{c}\text { Argonne } \\
\text { Pilot Test \# }\end{array}$ & $\begin{array}{c}\text { Contamination Surface } \\
\text { for Cs-137 }\end{array}$ & $\begin{array}{c}\text { Contaminant Aging } \\
\text { Test Conditions }\end{array}$ & $\begin{array}{c}\text { Contaminant } \\
\text { Aging Time } \\
\text { (days) }\end{array}$ & $\begin{array}{c}\text { Decon Gel } \\
\text { Application }\end{array}$ \\
\hline Pre-demo & $6 \times 6$ in. rough concrete & $22^{\circ} \mathrm{C}, 76 \% \mathrm{RH}$ & 3 & Hand trowel \\
1 & $6 \times 6$ in. rough concrete & $\begin{array}{c}40^{\circ} \mathrm{C}, 90 \% \mathrm{RH} \\
40^{\circ} \mathrm{C}, 70-90 \% \mathrm{RH}^{*} \\
22^{\circ} \mathrm{C}, 70-90 \% \mathrm{RH}^{*}\end{array}$ & $\begin{array}{c}2^{*} \\
2^{*}\end{array}$ & Hand trowel \\
& & & $7^{*}$ & Hand trowel \\
2 & $6 \times 6$ in. smooth concrete & $22^{\circ} \mathrm{C}, 64 \% \mathrm{RH}^{*}$ & & Spray gun \\
\hline
\end{tabular}

* Technical issues were noted with the humidity chamber and the system remained unoperational for the noted duration of tests. The low water vapor permeability of the lowdensity polyethylene bags used for the coupon tests [8] was used as the basis for assuming that the relative humidity for the coupons was the average relative humidity for the day when the coupons were placed into the bags.

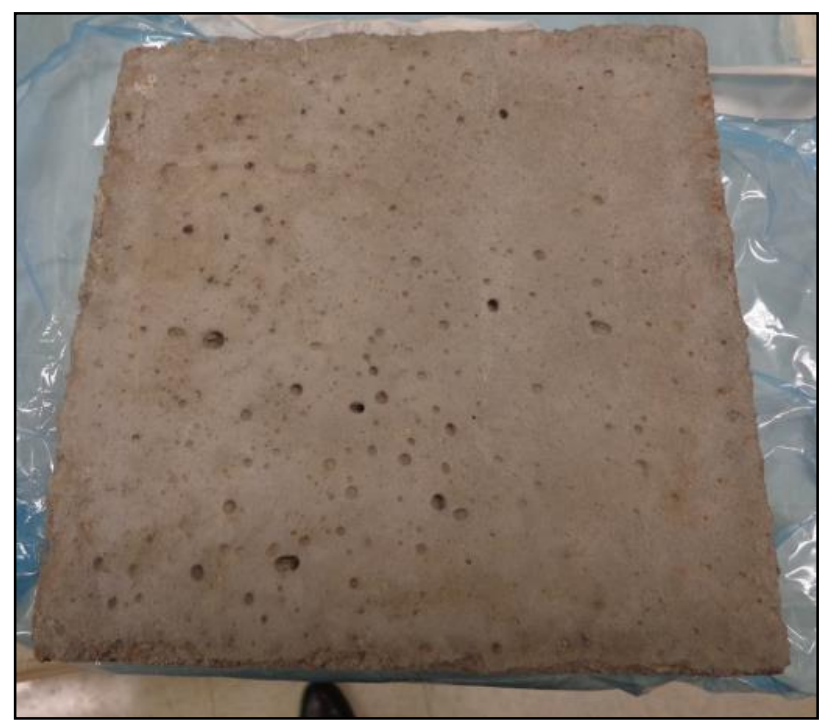

FIGURE 5-3 Photograph showing large pores on surface of concrete coupon. 
Non-radioactive and radioactive dry runs with a $6 \times 6$ in. test sample were performed to evaluate best practices and to ensure a smooth demonstration. A test sample was securely attached to the test stand, and the gel prepared above was applied to the face of the test sample with a plastic trowel.

On the day of the demonstration, test samples were removed from the humidity chamber or double-bag containment and placed onto the test stand. The test samples were placed numerically in rows from left to right. The gel was applied to all test samples, except Sample 5 (control sample), using a trowel spackling tool (Figures 5-4 and 5-5). Gel was applied to the face of each test sample beginning with Sample 1 through Sample 9 and was completed in four min. After one $\mathrm{h}$, the gel was removed from the coupons with the 3-in.-diameter hose of the HEPA vacuum (Figure 5-6). The hydrogel was removed cleanly with very little residue (Figure 5-7), although it should be noted that the suction from a portable wet/dry vacuum is stronger. Test samples were removed from the test stand, double-bagged, and surveyed for transfer to the gamma counting room.

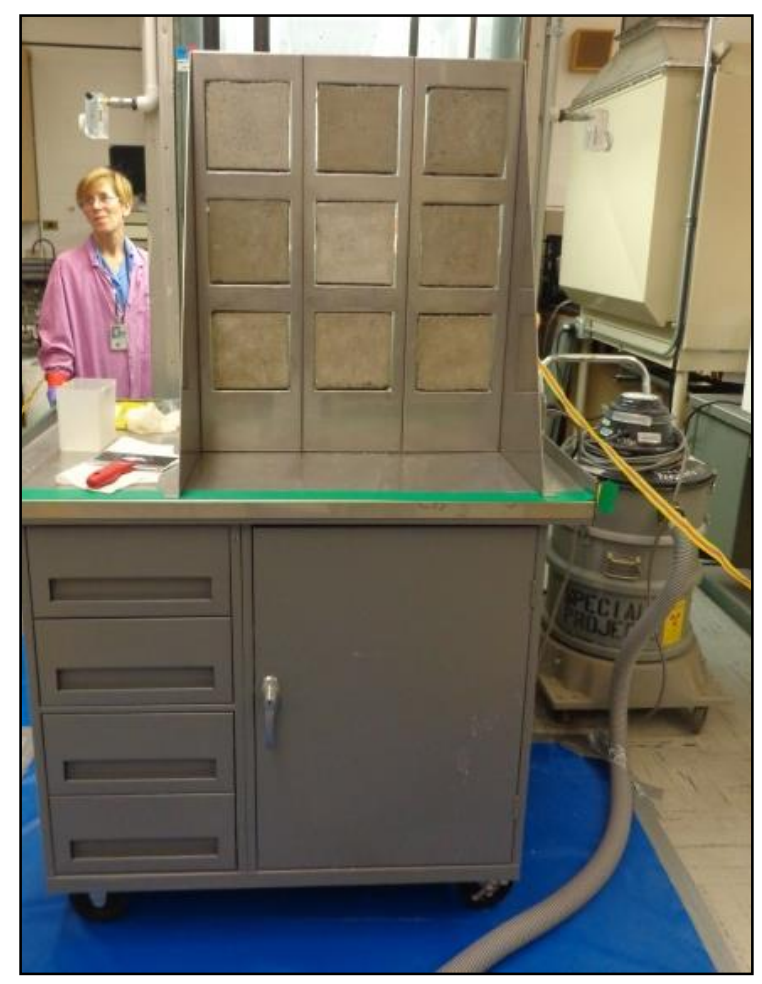

FIGURE 5-4 Photograph of demonstration test stand and setup: attached coupons, trowel for 1.0 M KCl gel application (on left side of test stand), and HEPA-Vac for gel removal (on floor to right of cart). 


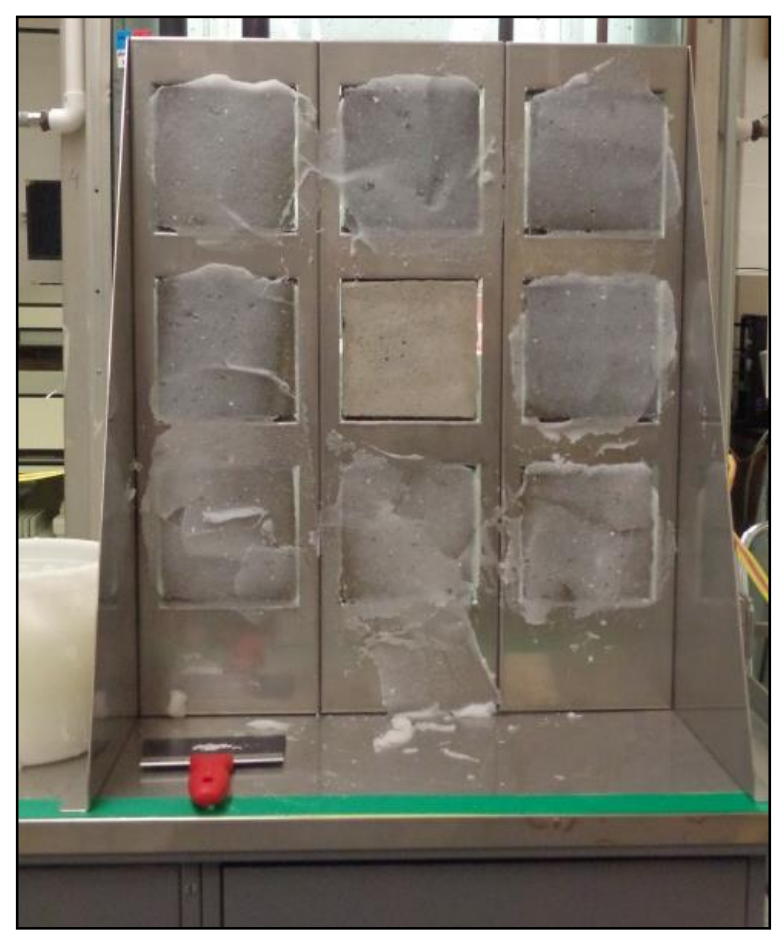

FIGURE 5-5 Photograph of test sample decontamination using 1.0 M KCl gel formulation for pilot test \#1.

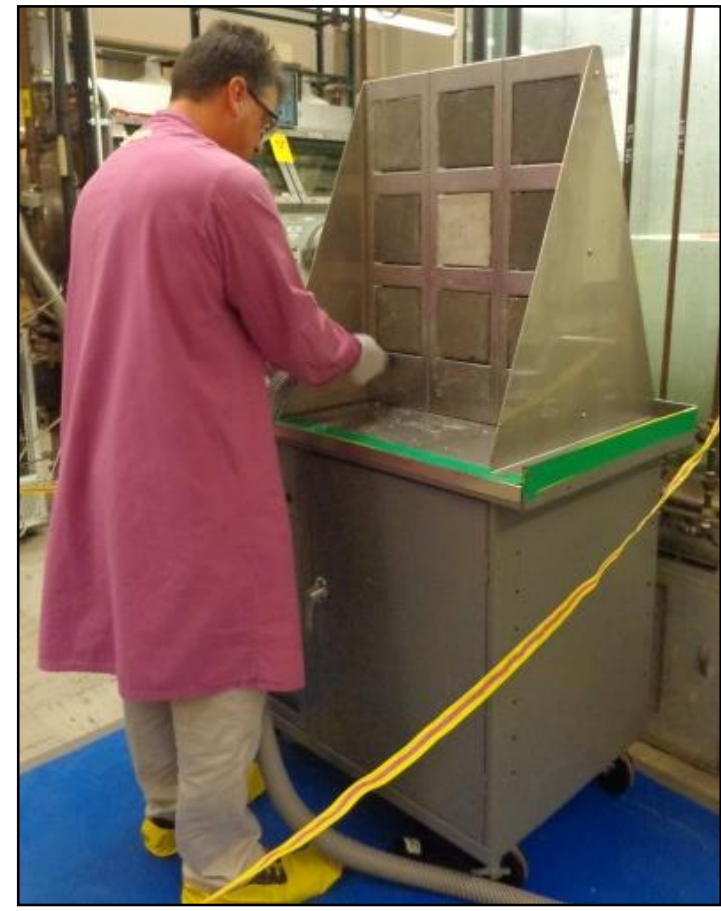

FIGURE 5-6 Photograph showing 1.0 M KCl gel removal with HEPA-Vac. 


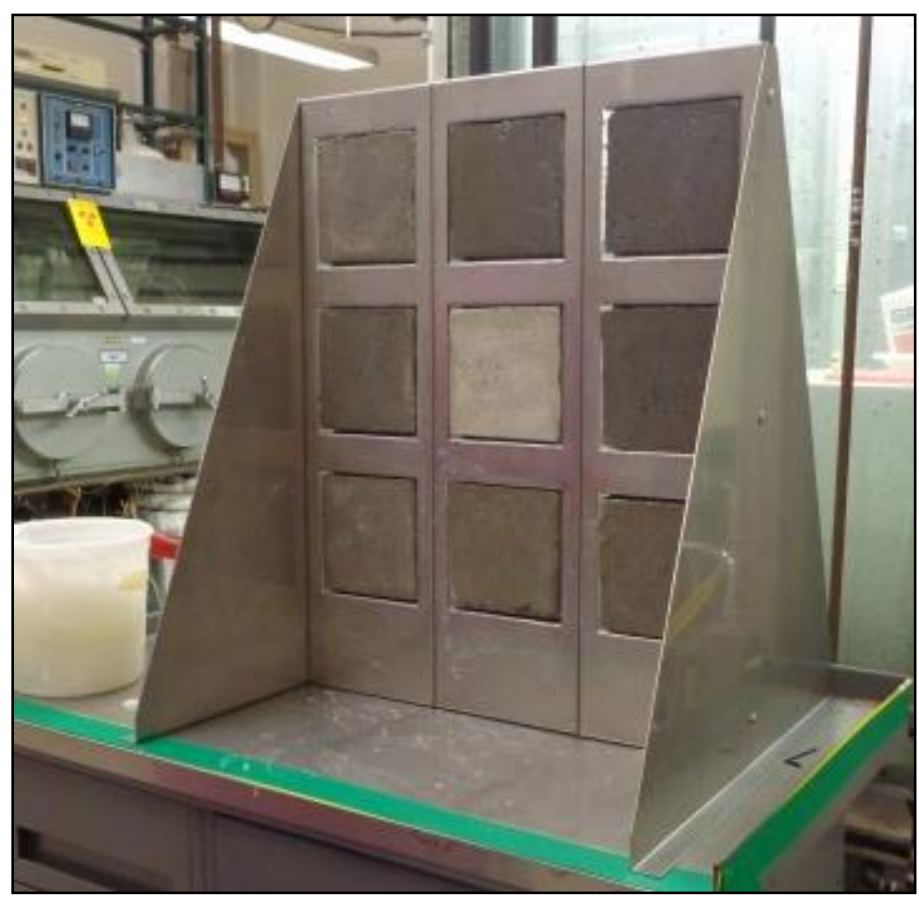

FIGURE 5-7 Photograph of completed demonstration from pilot test \#1.

In Argonne Pilot Test\#2, we repeated the demonstration by contaminating the smooth coupon face (Figure 5-8). Eight unused samples were contaminated on the smooth front side, as described above. The contaminated control coupon (coupon \#5) from Pilot Test \#1 was reused. Because the humidity chamber was still not operational and undergoing repairs, it was not used for aging the coupons. The Cs-137 contaminated coupons remained within double-bag containment at ambient temperature for one week.

The $1.0 \mathrm{M} \mathrm{KCl}$ gel formulation was used for the decontamination. The test procedure was the same as described for Pilot Test \#1. The SuperGel was applied by hand using a trowel. The test stand with gel applied to the coupons is shown in Figure 5-9. After one hour, the gel was removed from the coupons with the 3 -in. diameter hose of the HEPA vacuum. 


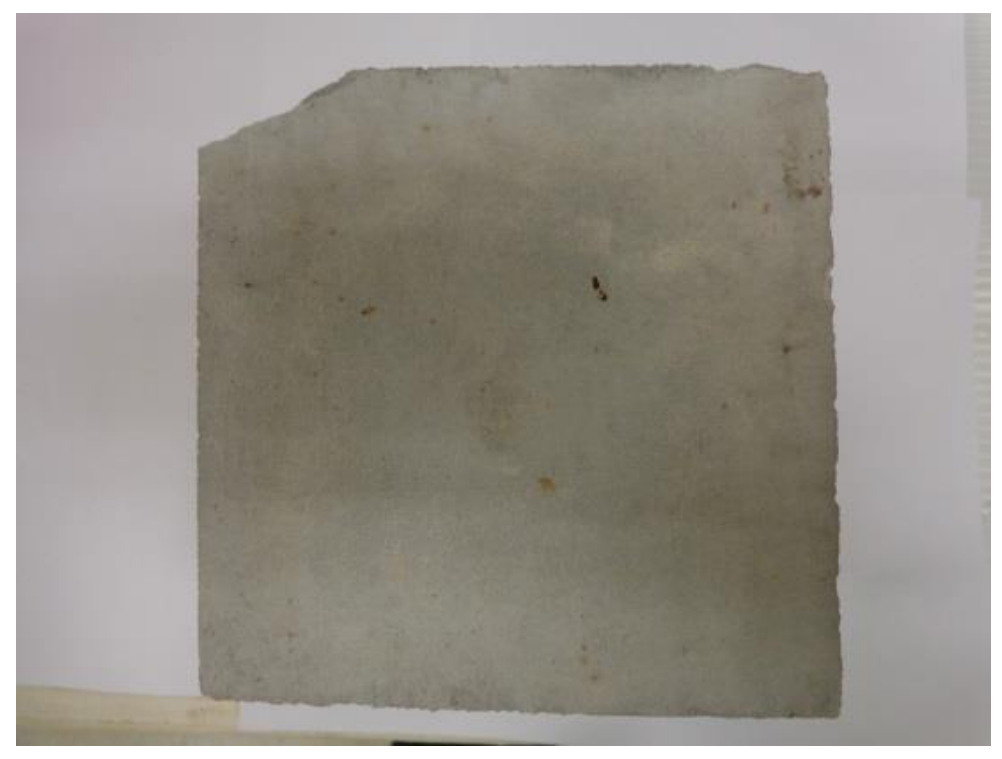

FIGURE 5-8 Photograph showing smooth side of concrete coupon used in pilot test $\# 2$.

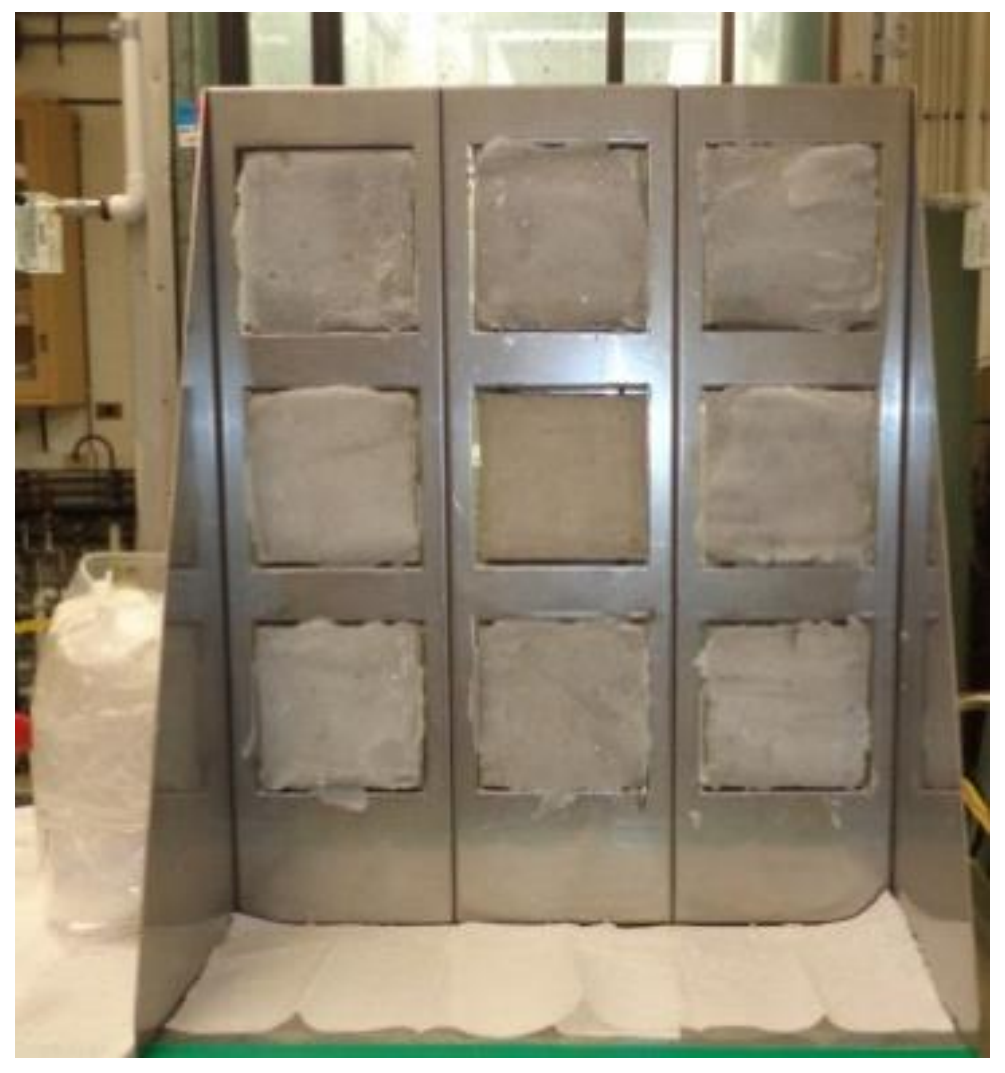

FIGURE 5-9 Photograph of coupon decontamination (smooth side) using 1.0 M KCl gel formulation for pilot test \#2. 
In Argonne Pilot Test\#3, the nine coupons used in Test \#1 (contaminated on the rough, backside) were reused. To start, the front-side (smooth) face was gamma counted to establish the background due to the residual contamination on the (rough) backside. The front side was then contaminated as described above and gamma counted again (except for coupon \#5). Contaminated coupons were aged for one week in the humidity chamber at $90 \%$ relative humidity and $40^{\circ} \mathrm{C}$. Decontamination of the coupons was performed in a tented shelter (Figure 5-10). The gel was applied using a viscous spray unit (GRACO 55:1 Bulldog, 5-gallon stationary single-post ram package with GRACO airless gun) (Figures 5-11 and 5-12). After one hour, the gel was removed from the coupons with the 3-in. diameter hose of the HEPA vacuum (Figure 5-13).

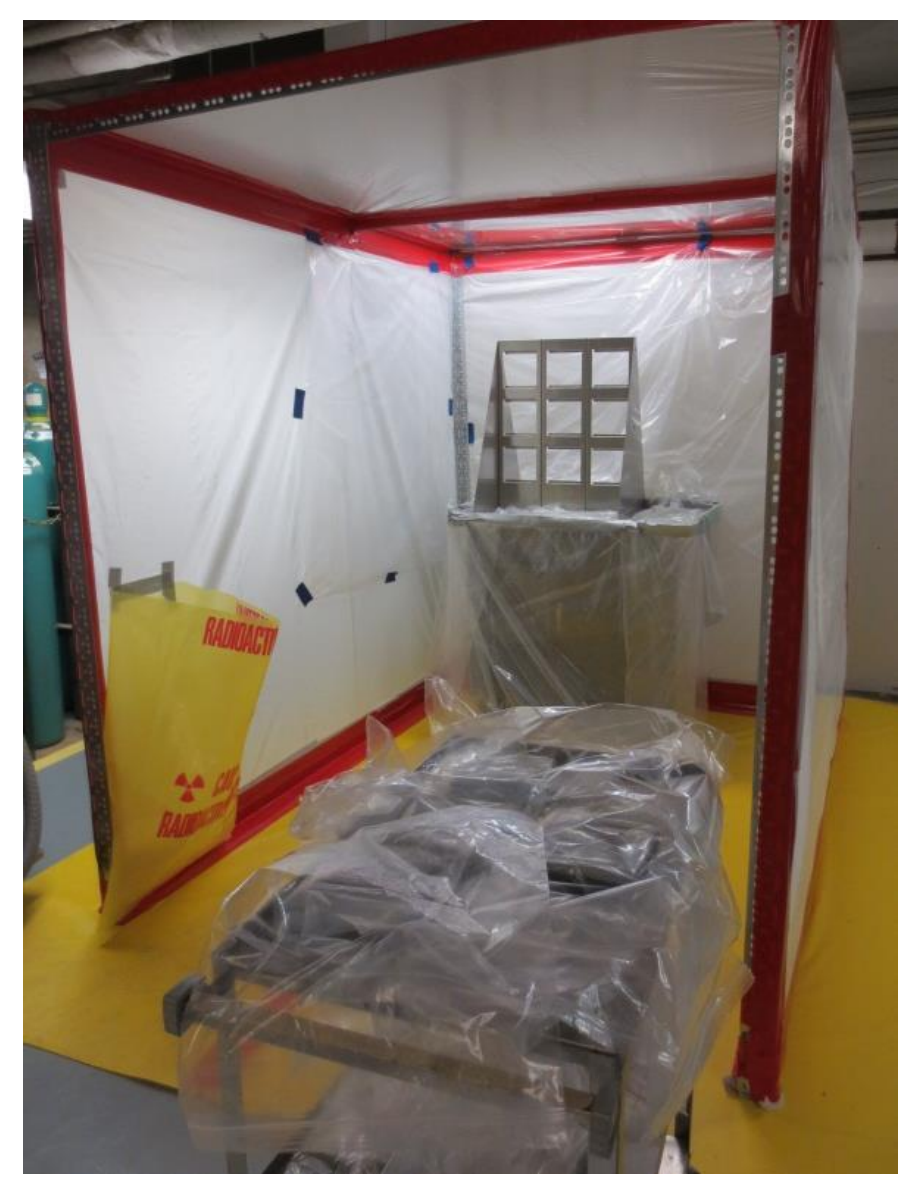

FIGURE 5-10 Tented contamination shelter for Argonne pilot test \#3 with test stand in back of the shelter and double-bagged coupons on cart in front opening of shelter. 


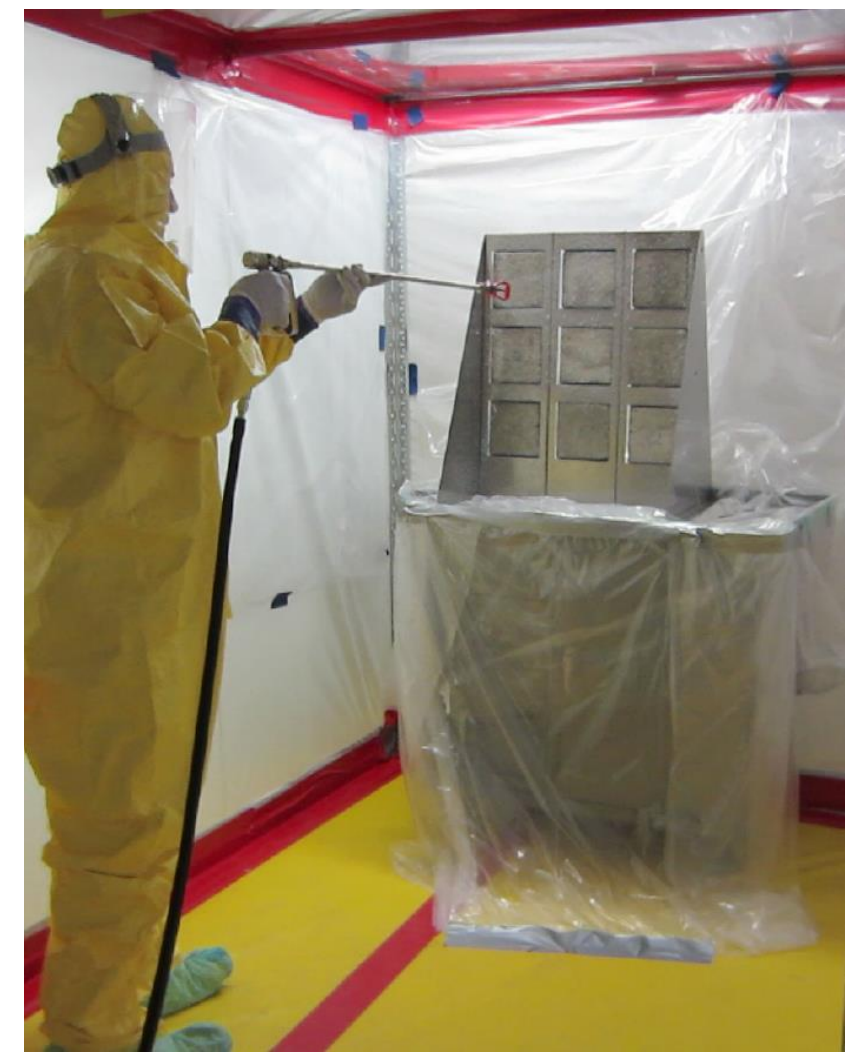

FIGURE 5-11 Sprayer wand ready to start gel application on contaminated coupons secured within test stand in tented shelter.

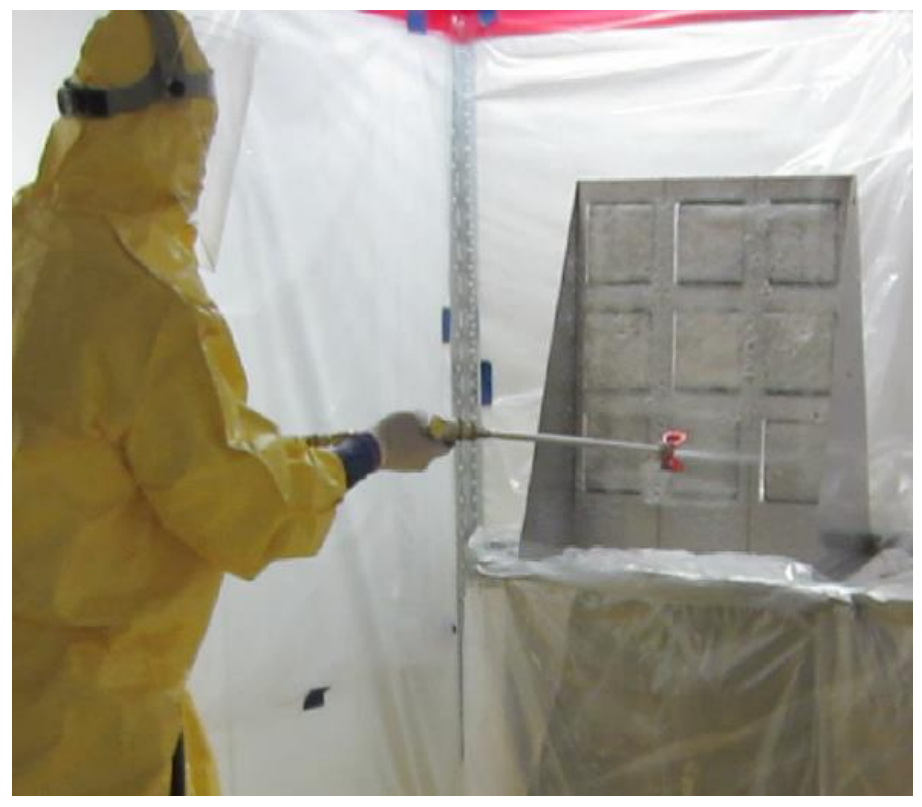

FIGURE 5-12 Sprayer applying gel to contaminated coupons. 


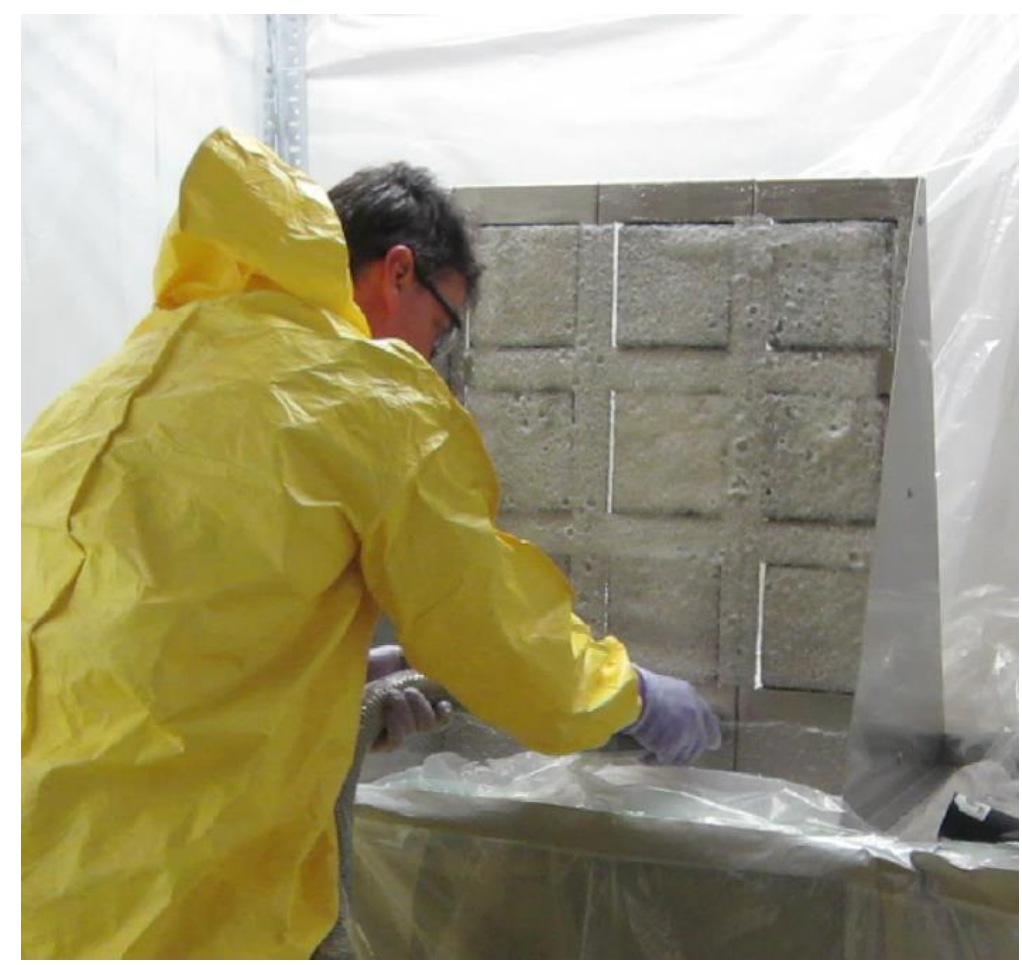

FIGURE 5-13 Removal of gel from contaminated coupons using HEPA vacuum during pilot test \#3.

\subsection{RESULTS}

\subsubsection{Density and Porosity Measurements}

The true density of the concrete pieces received from the sponsor for the Argonne Pilot Tests $\left(m_{\text {concrete }}=4.0872 \mathrm{~g}\right.$ ) was measured by pycnometer with the following values:

$$
\begin{aligned}
& \text { Run \#1: } \mathrm{P}_{1}=16.983, \mathrm{P}_{2}=8.067, \mathrm{~V}_{\text {concrete }}=0.9999 \mathrm{~cm}^{3} \\
& \text { Run \#2: } \mathrm{P}_{1}=17.112, \mathrm{P}_{2}=8.128, \mathrm{~V}_{\text {concrete }}=0.9994 \mathrm{~cm}^{3}
\end{aligned}
$$

This gave a true density of $2.596 \mathrm{~g} / \mathrm{cm}^{3}$.

Two measurements using Archimedes method on $2.5949 \mathrm{~g}$ of concrete yielded masses of displaced water of $1.1484 \mathrm{~g}$ and $1.083 \mathrm{~g}$ for an average volume of water of $1.1157 \mathrm{~g}$. With the density of water of $0.999 \mathrm{~g} / \mathrm{cm}^{3}$, a bulk density of $2.33 \mathrm{~g} / \mathrm{cm}^{3}$ was calculated. Using these two density values, we calculated a porosity of $11 \%$, compared to $24 \%$ for the small coupons used throughout the program. This low porosity for the large concrete coupons seems contradictory to observations made during contamination of the coupons, whereby the contaminant solution beaded up on the surface on the small coupons and took almost an hour to dry versus the immediate uptake of the contaminant solution by the large concrete coupons. Without details on 
the fabrication of the coupons it is difficult to conjecture the discrepancy in density between small lab samples and these pilot scale test samples. Perhaps hydrophobic polymer was included in the small coupon tests that were not in the larger coupons. Such polymers are added often to concrete to control properties. Such a polymer could permit higher decontamination factors than more dense coupons.

\subsubsection{Idaho Testing of Combined Formulation}

Idaho National Laboratory (INL) conducted tests on the removal of americium-243 from U.S. concrete samples (Table 5-2). Even though the contaminated substrates were U.S.-made concrete $(6 \times 6$ in. coupons $)$ with a very different composition than that of concrete reported in previous sections of this report, this is valuable data since it provides another test set for the mixed isotope hydrogel formulation.

TABLE 5-2 Preliminary Results from INL Pilot Test on U.S. Concrete Coupons.

\begin{tabular}{lccc}
\hline & $\begin{array}{c}\text { Pre-Decon } \\
\text { Activity } \\
\text { (nCi/Coupon) }\end{array}$ & $\begin{array}{c}\text { Post-Decon } \\
\text { Activity } \\
\text { (nCi/Coupon) }\end{array}$ & $\%$ RSD \\
\hline & 52 & 10 & $80 \%$ \\
& 56 & 21 & $62 \%$ \\
& 51 & 18 & $65 \%$ \\
& 42 & 16 & $62 \%$ \\
Avg & 50 & 17 & $67 \%$ \\
SD & 6 & 5 & $9 \%$ \\
\hline
\end{tabular}

\subsubsection{Argonne Testing of Combined Gel Formulation}

The pre-test for Argonne Pilot Test \#1 was performed on a single concrete coupon to establish safe work procedures. Therefore, this coupon was aged under ambient lab conditions and was not maintained under the test conditions for the pilot test \#1 coupons. This pre-test coupon was only aged for 3 days in double-bag containment at $22^{\circ} \mathrm{C}$ and $76 \% \mathrm{RH}$ (ambient conditions). Decontamination of the coupon resulted in $52.4 \%$ cesium removal from its rough surface.

Pilot Test \#1 demonstration coupons were aged for 7 days within the humidity chamber. The chamber failed to maintain the set humidity during the coupon aging process, but testing was continued within the chamber while we attempted to maintain high humidity conditions by using open containers of water (refer to Table 5-1). The contamination and decontamination data for the coupons are shown in Figure 5-14. Triplicate gamma counting was performed on the 
individual coupons and showed very little variability, with a RSD of $0.2-1.7 \%$. The positive control coupon validated the gamma counting and decontamination test processes, i.e., no effect on the control coupon activity was found (RSD of $0.7 \%$ for the control counts before and after decontamination). Background counts of a clean coupon were also performed and gave low count rates expected for the counting facility. Decontamination results for removal of cesium from Pilot Test \#1 on concrete coupons with a rough surface are shown in Figure 5-15. The average cesium removal was $31.7 \%$ ( $\pm 3.7 \%$ standard deviation) for coupons that were aged for 7 days at $22-40^{\circ} \mathrm{C}$ and $70-90 \% \mathrm{RH}$. The RSD between the coupons tested was only $12 \%$ despite the variability in test aging conditions.

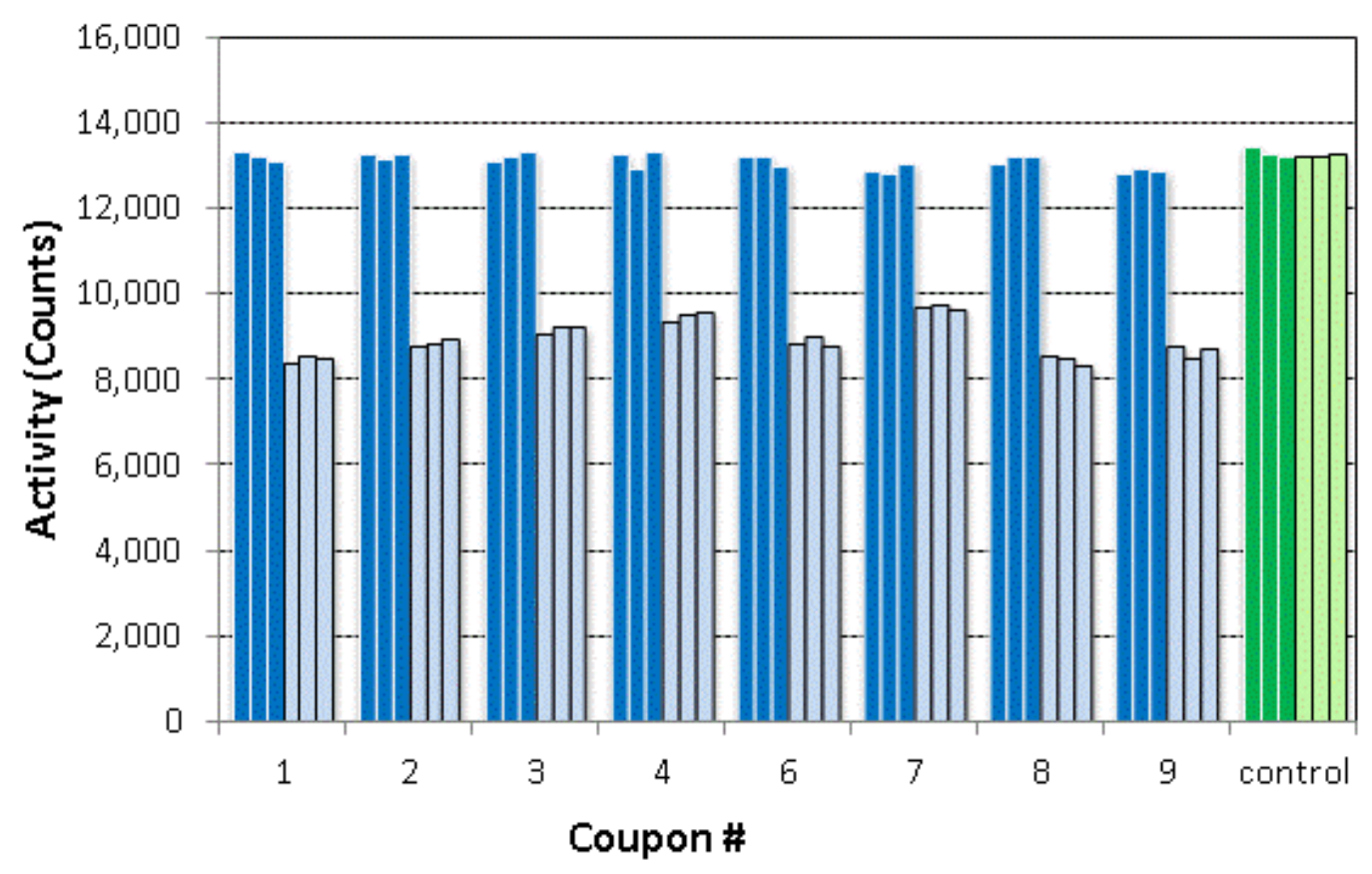

FIGURE 5-14 Activity of coupons from Argonne Pilot Test \#1 before (dark color) and after (light color) decontamination. Triplicate counts shown for individual coupons; control coupon activity shown on right. 


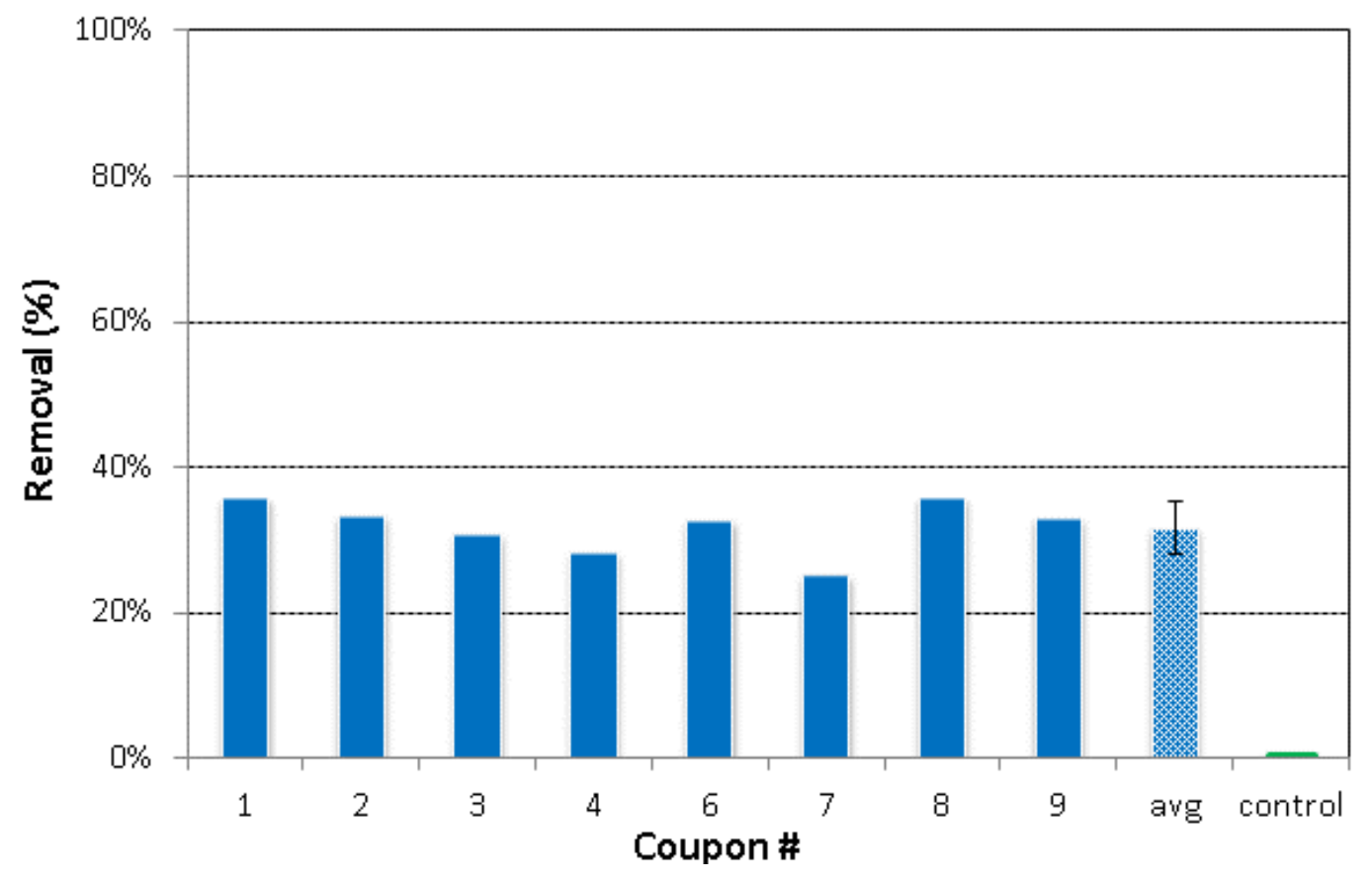

FIGURE 5-15 Decontamination results for Cs-137 from Argonne Pilot Test \#1.

The smooth surfaces of concrete coupons were contaminated with Cs-137 in the Pilot Test \#2 demonstration. Coupons were aged for 7 days within double-bag containment at ambient room conditions. At the time of this demonstration, the humidity chamber was not operational and undergoing repairs. The contamination and decontamination data for the coupons are shown in Figure 5-16. Triplicate gamma counting was performed on the individual coupons and showed very little variability (RSD of $0.2-3.0 \%$ ). The positive control coupon validated the gamma counting and decontamination test processes, i.e., no effect on the control coupon activity was found (RSD of $4.0 \%$ for the control counts before and after decontamination). Background counts of a clean coupon were also performed and gave low count rates expected for the counting facility. Decontamination results for removal of cesium from Pilot Test \#2 on concrete coupons with a smooth surface are shown in Figure 5-17. The average cesium removal was $50.5 \%$ ( $\pm 5.7 \%$ RSD) for coupons that were aged for 7 days at $22^{\circ} \mathrm{C}$ and $64 \% \mathrm{RH}$. The RSD for the decontamination for all eight coupons tested was only $11 \%$. While cesium was removed 1.6 times greater from the smooth concrete surface than the rough surface, the coupon aging conditions were not the same between pilot test \#1 and \#2. An additional pilot test was performed on concrete coupons at the required temperature and humidity for cesium contaminant on the smooth surface. 


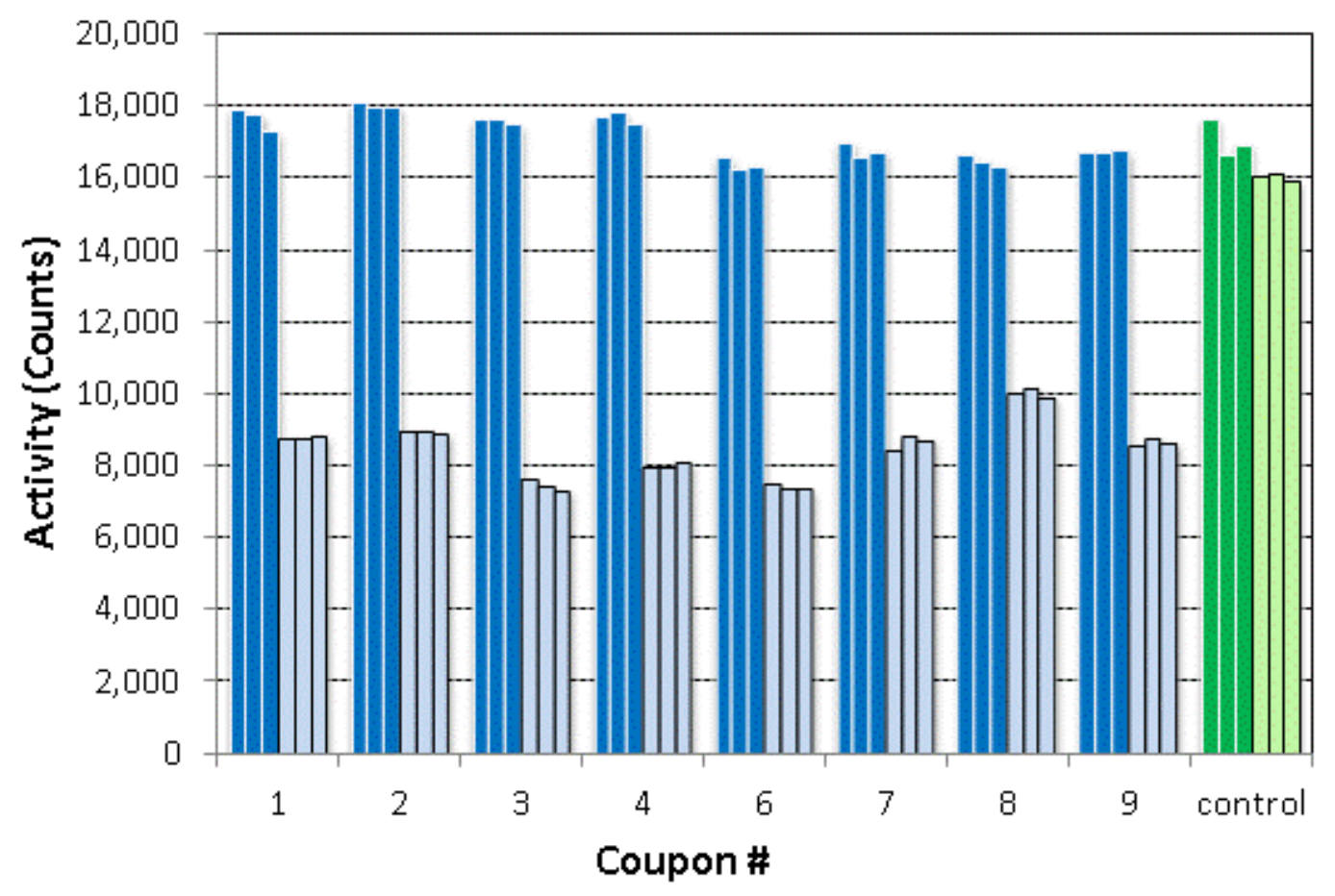

FIGURE 5-16 Activity of coupons before (dark color) and after (light color) decontamination from Argonne Pilot Test \#2. Triplicate counts shown for individual coupons; control coupon activity shown on right.

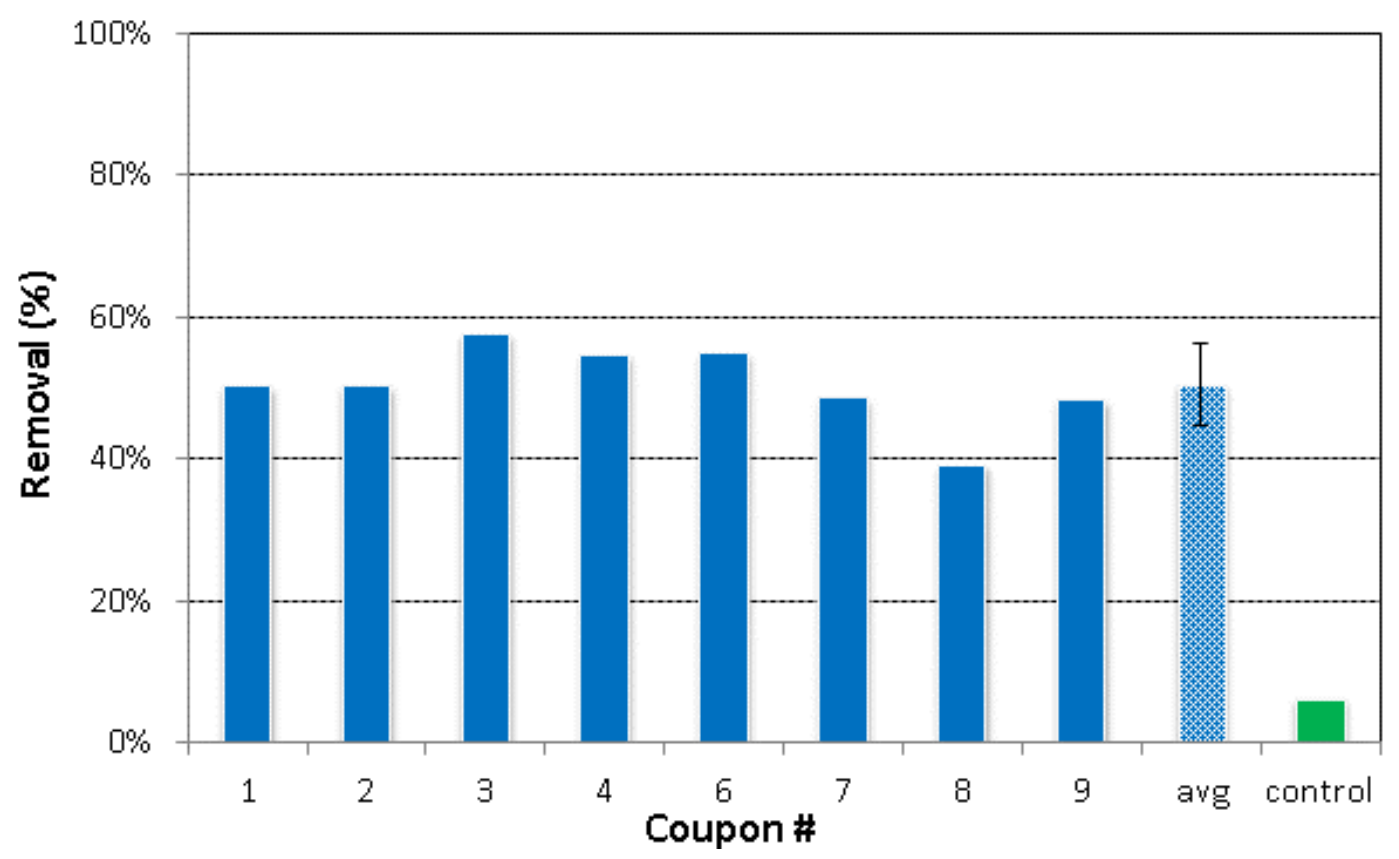

FIGURE 5-17 Decontamination results for Cs-137 from Argonne Pilot Test \#2 (individual coupons and average of eight coupons and control coupon). 
The smooth surfaces of concrete coupons were contaminated with Cs-137 in the Pilot Test \#3 demonstration. The coupons were aged for 7 days within the humidity chamber at $40^{\circ} \mathrm{C}$ and $90 \% \mathrm{RH}$. The contamination and decontamination data for the coupons are shown in Figure 5-18. Triplicate gamma counting was performed on the individual coupons and showed very little variability (RSD of $1.7 \%$ ). The positive control coupon validated the gamma counting and decontamination test processes, i.e., no effect on the control coupon activity was found (RSD of $1.8 \%$ for the control counts before and after decontamination). Background counts of a clean coupon were also performed and gave low count rates expected for the counting facility. Decontamination results for removal of cesium from Pilot Test \#3 on concrete coupons with a smooth surface are shown in Figure 5-19. The average cesium removal was 36.8\% $\left( \pm 4.0 \%\right.$ standard deviation) for coupons that were aged for 7 days at $40{ }^{\circ} \mathrm{C}$ and $90 \% \mathrm{RH}$. The relative standard deviation for the decontamination for all eight coupons tested was low, $11 \%$, which is typical of what had been seen with other tests.

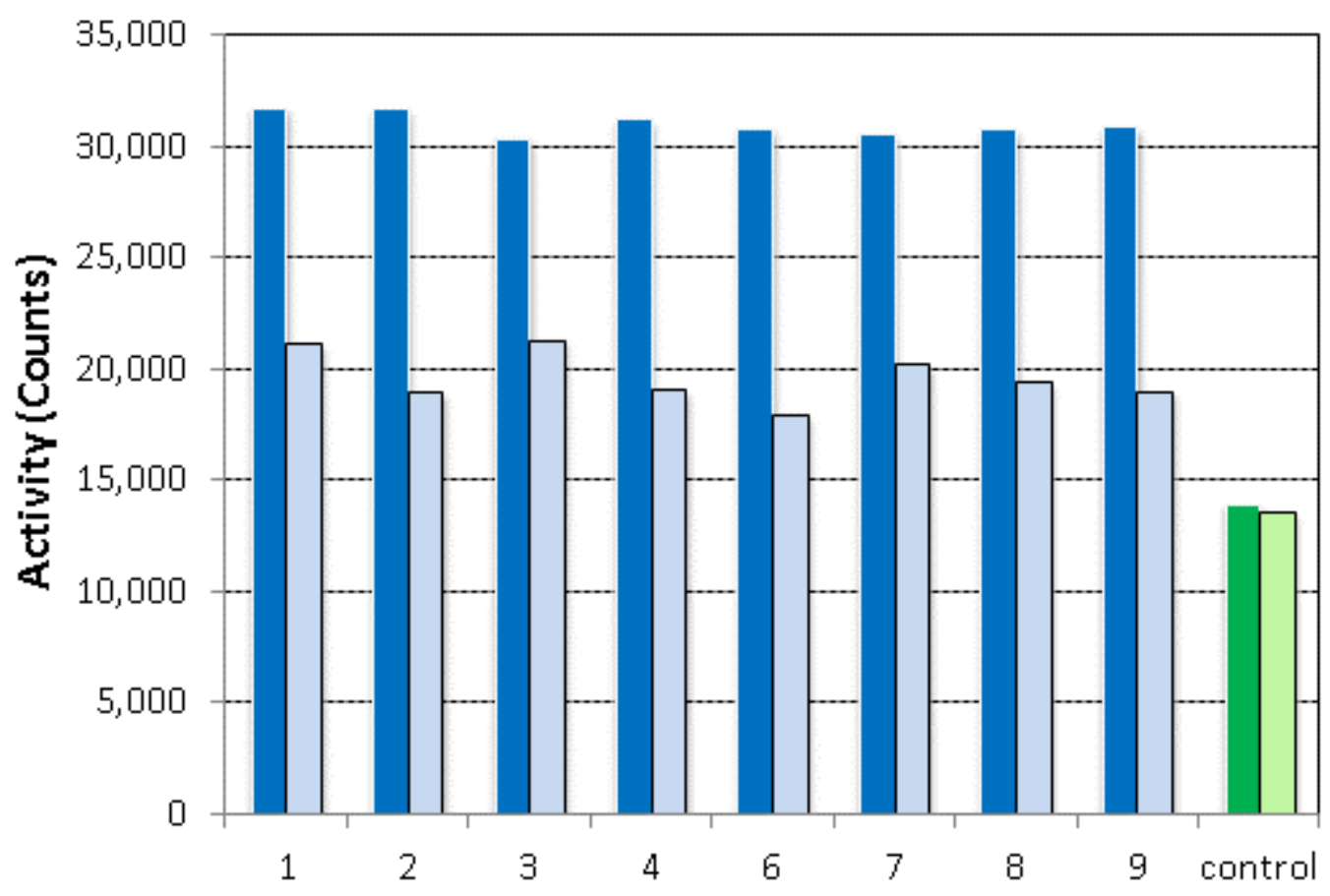

\section{Coupon \#}

FIGURE 5-18 Activity of coupons before (dark color) and after (light color) decontamination from Argonne Pilot Test \#3. Triplicate counts shown for individual coupons; control coupon activity shown on right. 


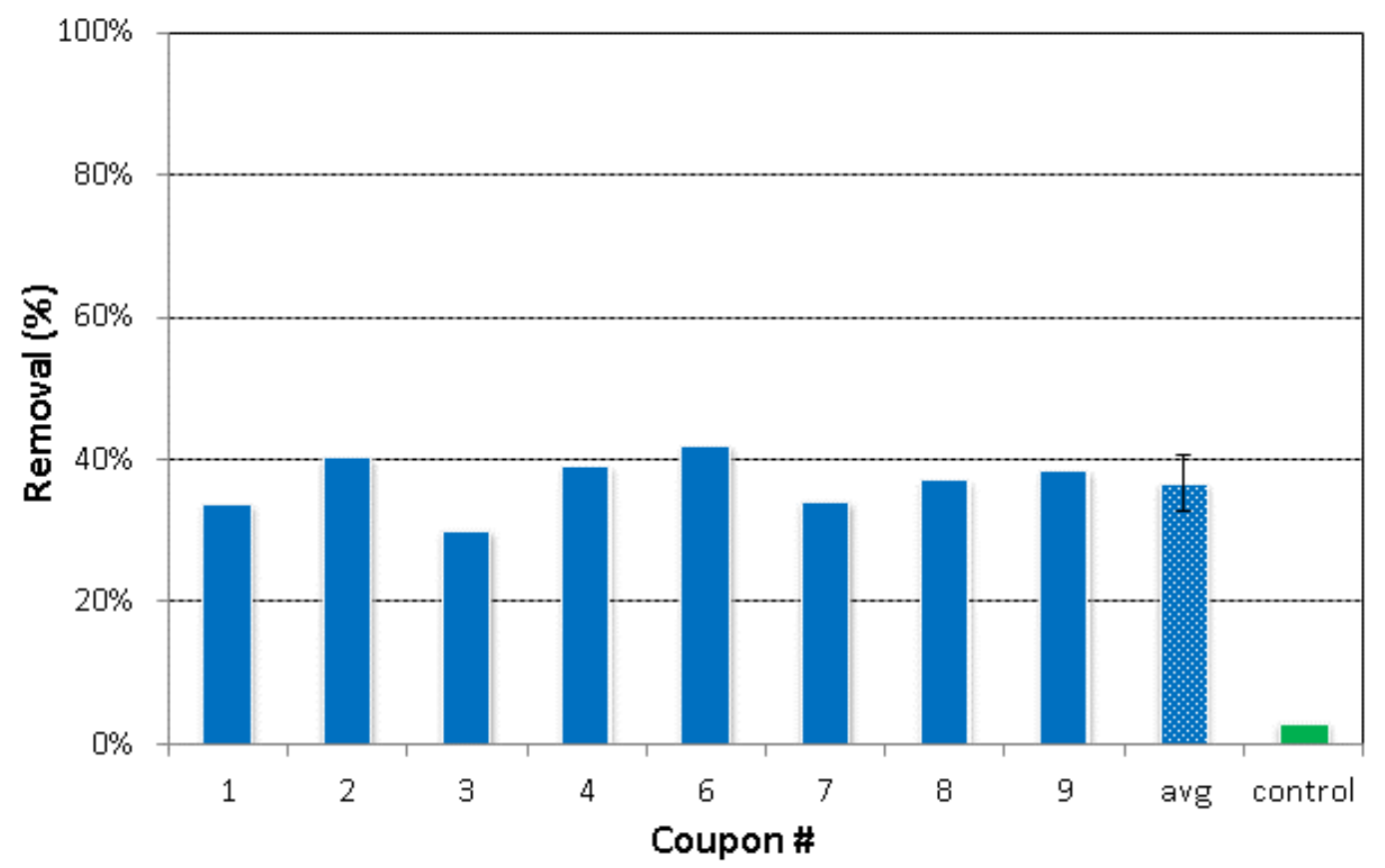

FIGURE 5-19 Decontamination results for Cs-137 from Argonne Pilot Test \#3 (individual coupons and average of eight coupons and control coupon).

While it was initially assumed that the short aging time (pre-test with only one coupon) and surface roughness of the coupons (large pores in the coupon face) were a key factor in lower decontamination results when compared with the small coupon testing results, higher temperatures $\left(40^{\circ} \mathrm{C}\right)$ and low humidity for aging the contaminant may also be important. The average cesium removal was slightly higher (but within the standard deviation of the results) from the smooth concrete surface than the rough surface when the coupon aging conditions were most similar (Table 5-3 for pilot tests \#1 and \#3). When coupon surfaces were the same (smooth), the lower temperature and humidity aging conditions for the contaminant on the concrete gave $13.7 \%$ higher cesium removal (Table 5-3 for pilot tests \#2 and 3).

Future work could entail characterization of the composition of these $6 \times 6$ in. coupons was identical to the $2 \times 2$ in. coupons previously provided, of the pore structure on each side of the coupon and testing of surface absorption, and permeability as a function of aging conditions. 
TABLE 5-3 Summary of cesium removal results on Singapore concrete from Argonne pilot tests.

\begin{tabular}{|c|c|c|c|c|}
\hline $\begin{array}{c}\text { Argonne } \\
\text { Pilot Test \# }\end{array}$ & $\begin{array}{c}\text { Contaminant Aging } \\
\text { Conditions }\end{array}$ & $\begin{array}{c}\text { Coupon } \\
\text { Contamination } \\
\text { Surface } \\
\end{array}$ & $\begin{array}{l}\text { Number of } \\
\text { Contaminated } \\
\text { Coupons } \\
\text { Tested } \\
\end{array}$ & $\begin{array}{c}\% \text { Cs } \\
\text { Removal } \\
\text { Average } \\
\text { (Standard } \\
\text { Deviation) }\end{array}$ \\
\hline Pre-demo & $\begin{array}{l}\text { Time: } 3 \text { days } \\
\text { Containment: double-bag } \\
\text { Temp: } 22^{\circ} \mathrm{C}^{\mathrm{a}} \\
\text { Relative Humidity: } 76 \%^{\mathrm{b}}\end{array}$ & rough & 1 & 52.4 \\
\hline 1 & $\begin{array}{l}\text { Time: } 7 \text { days } \\
\text { Containment: chamber } \\
\text { Temp: } 22^{\mathrm{a}}-40^{\circ} \mathrm{C} \\
\text { Relative Humidity: } 70^{\mathrm{c}}-90 \%\end{array}$ & rough & 8 & $31.7( \pm 3.7)$ \\
\hline 2 & $\begin{array}{l}\text { Time: } 7 \text { days } \\
\text { Containment: double-bag } \\
\text { Temp: } 22^{\circ} \mathrm{C}^{\mathrm{a}} \\
\text { Relative Humidity: } 64 \%^{\mathrm{b}}\end{array}$ & smooth & 8 & $50.5( \pm 5.7)$ \\
\hline 3 & $\begin{array}{l}\text { Time: } 7 \text { days } \\
\text { Containment: chamber } \\
\text { Temp: } 40^{\circ} \mathrm{C} \\
\text { Relative Humidity: } 90 \%\end{array}$ & smooth & 8 & $36.8( \pm 4.0)$ \\
\hline
\end{tabular}

a Assumed average ambient laboratory temperature.

b Assumed average relative humidity for day coupons that were bagged; information obtained from local city weather history records.

c Chamber failure occurred.

\section{CONCLUSIONS}

Argonne National Laboratory improved the superabsorbing gel-based process (SuperGel) for the decontamination of cesium from concrete and other porous building materials from a coastal city in Southeast Asia. To mitigate a potential problem in obtaining bulk quantities of crystalline silico-titanate materials (IONSIV-910) for capture of cesium, we perused the literature for other sequestering agents. There was little information in the literature describing the sorption characteristics of cesium from salty waters except for the work done related to the underground, high-level liquid waste storage tanks in the United States that contain molarity concentrations of sodium. We chose to test the clays illite and vermiculite with the zeolite chabazite. Chabazite and vermiculite did not produce $\mathrm{K}_{\mathrm{d}}$ values as high as crystalline silicotitanate in $\mathrm{NH}_{4}{ }^{+}$salt solutions but did exceed the $\mathrm{K}_{\mathrm{d}}$ values for crystalline silico-titanate in 
$\mathrm{K}^{+}$solutions. Both chabazite and vermiculite are available in bulk quantities from the United States but their availability worldwide would have to be determined.

Similarly, we explored sequestering agents for Am3+ and found that the literature does not include separation from salty solutions except for the studies on liquid nuclear waste solution. We decided to test birnessite and cliniptilolite and compare these results to those for sorption onto montmorillonite and vermiculite. From KCL, $\mathrm{Am}^{3+}$ sorbed well onto all but montmorillonite. In the acidic HEDPA solutions, all sorbents had $\mathrm{K}_{\mathrm{d}}$ values $<20 \mathrm{~mL} / \mathrm{g}$. In Na ${ }^{+}$, birnessite performed well above $\left(\mathrm{K}_{\mathrm{d}}>10,000 \mathrm{~mL} / \mathrm{g}\right)$ the others $\left(\mathrm{K}_{\mathrm{d}}<200 \mathrm{~mL} / \mathrm{g}\right)$. The availability of bulk birnessite needs further evaluation.

Next, we tested the SuperGel formulation that was compatible for both cesium and americium decontamination. This formulation combined the $\mathrm{K}^{+}$or $\mathrm{NH}_{4}{ }^{+}$salts needed for cesium decontamination with the $\mathrm{CO}_{3}{ }^{2-}$ and HEDPA needed to decontaminate americium effectively. We tested different combinations of these reagents and found that 64 to $83 \%$ Cs-137 could be removed with no difference in percent removal of cesium as a function of concentration of cesium for coupons aged for 24 hours with contamination. The most effective combination was the $1 \mathrm{M} \mathrm{K}^{+}$or $\mathrm{NH}_{4}{ }^{+}$as the carbonate salt in $0.025 \mathrm{M}$ HEDPA. We tested the removal of americium and cesium from concrete, brick, and tile aged one week with contamination. For cesium tests, we removed $73 \%$ from tile, $45 \%$ from concrete and $9.8 \%$ from brick. For americium tests, we removed $86 \%$ from tile, $31 \%$ from concrete, and $9.8 \%$ from brick. The lower percent removal values from previous tests is attributed to dry conditions during the aging process.

We conducted tests on U.S.-made $6 \times 6$ in. coupons $(1$ in. thick) contaminated with Cs-137 and aged for three days using the combined gel formulation $\left(0.5 \mathrm{M} \mathrm{K}_{2} \mathrm{CO}_{3}\right.$, 0.025 M HEDPA, in 99/1 cross linked to linear at 100\% hydration). From four replicates, they removed $67 \pm 9 \%$ of the cesium.

Then, we ran several tests on concrete samples ( 6 in. $\times 6$ in.) provided by the sponsor and aged the Cs-137 for seven days before applying the hydrogel reconstituted in $1 \mathrm{M} \mathrm{KCl}$. We removed $32 \%$ of cesium from the rough backside of the concrete samples aged at 70-90\% relative humidity and $22-40^{\circ} \mathrm{C}, 50.5 \%$ from the smooth side aged at $64 \%$ relative humidity and $22^{\circ} \mathrm{C}$, and $37 \%$ of cesium from the smooth side aged at $90 \%$ relative humidity and $40^{\circ} \mathrm{C}$.

Additional tests with mixed isotope formulation are recommended to understand better the variance of decontamination efficacy with material property and large coupon tests should be conducted with the mixed isotope formulation. 


\section{APPENDIX A CLINIPTOLOLITE SUPPLIERS IN UNITED STATES}

U.S. Companies Producing or Selling Clinoptilolite

\begin{tabular}{|c|c|c|c|}
\hline Company & Mine Site & Zeolite Type & Web Site \\
\hline $\begin{array}{l}\text { Bear River Zeolite LLC } \\
\text { P.O. Box } 643 \\
47 \text { Cox Gulch Road } \\
\text { Thompson Falls, MT } 59873 \\
\text { 406-827-3523 }\end{array}$ & Idaho & K-rich Clinoptilolite & http://www.bearriverzeolite.com/ \\
\hline $\begin{array}{l}\text { KMI Zeolite Inc. } \\
3100 \text { Sandy Valley Road } \\
\text { Jean, NV 89019 } \\
\text { Phone: } 702-723-5415\end{array}$ & California & Clinoptilolite & http://www.kmizeolite.com/ \\
\hline $\begin{array}{l}\text { St. Cloud Mining Co. } \\
\text { P.O. Box } 196 \\
761 \text { Saint Cloud Road } \\
\text { Winston, NM } 87943 \\
(575) 743-5215\end{array}$ & New Mexico & Clinoptilolite & http://www.stcloudmining.com/ \\
\hline $\begin{array}{l}\text { Steelhead Specialty Minerals } \\
\text { Rock Pointe Corporate Center } \\
1212 \text { North Washington Street } \\
\text { Suite } 12 \\
\text { Spokane, WA } 99201 \\
\text { (509) 328-5685 }\end{array}$ & California & Clinoptilolite & www.steelheadspecialtyminerals.com \\
\hline $\begin{array}{l}\text { Teague Mineral Products } \\
1925 \text { Highway 201 South } \\
\text { Adrian, OR } 97901-9407 \\
\text { (541) 339-3940 }\end{array}$ & Oregon & Clinoptilolite & http://teaguemineralproducts.com/ \\
\hline $\begin{array}{l}\text { Zeotech Corp. } \\
6 \text { Mangham Court } \\
\text { Peralta, NM } 87042 \\
(505) 866-0301\end{array}$ & Texas & Clinoptilolite & http://www.zeotechcorp.com/ \\
\hline
\end{tabular}


APPENDIX B COMPANIES CONTACTED FOR AVAILABILITY OF BIRNESSITE, CLINOPTILONITE, ILLITE, AND ROMANECHITE

\begin{tabular}{|c|c|c|c|c|c|}
\hline Company & Product & Amount/Cost & Comment & Contact & Link \\
\hline $\begin{array}{l}\text { Minerological } \\
\text { Research } \\
\text { Company }\end{array}$ & all & & mineral collectors & $408-923-6800$ & http://minresco.com/ \\
\hline $\begin{array}{l}\text { Cowboy } \\
\text { Mining } \\
\text { Company }\end{array}$ & none & $\begin{array}{l}\$ 80 \text { per ton- } \\
\text { flatbed holds } \\
221 / 2 \text { tons }\end{array}$ & Have $\mathrm{Na}$ bentonite & $800-243-7455$ & www.cowboymining.com/ \\
\hline $\begin{array}{l}\text { American } \\
\text { Colloid } \\
\text { Company }\end{array}$ & all & & $\begin{array}{l}\text { Spoke to geologist/mining } \\
\text { manager: see file for USA } \\
\text { Zeolite Sources-said to try } \\
\text { Boral, KMI, and St. Cloud } \\
\text { for cliniptilolite (below). }\end{array}$ & $\begin{array}{l}\text { Dave Carroll - } \\
\text { Geologist/Mining } \\
\text { Manager }\end{array}$ & www.colloid.com/ \\
\hline Boral & illite & & $\begin{array}{l}\text { Spoke to Carl and will get } \\
\text { back to me. }\end{array}$ & $800-5$ boral 5 & www.boralbricks.com \\
\hline
\end{tabular}




\begin{tabular}{|c|c|c|c|c|c|c|}
\hline Company---- & Product & Amount/Cost & Comment & Contact & Link & \\
\hline $\begin{array}{l}\text { St. Cloud } \\
\text { Mining Co. } \\
\text { (on list from } \\
\text { American } \\
\text { Colloid) }\end{array}$ & clinoptilolite & $\$ 450$ per ton & $\begin{array}{l}\text { Largest supplier of } \\
\text { cliniptilolite in U.S.; } \\
\text { however, it is made to } \\
\text { order. Sent us } 2 \text { types each } \\
\text { with } 3 \text { mesh sizes plus a } \\
\text { sample of chabazite. }\end{array}$ & $\begin{array}{l}\text { Joe McEnaney } \\
\text { 203-761-1291 } \\
\text { cell 203-209-6084 }\end{array}$ & http://stcloudmining.com/ & \\
\hline $\begin{array}{l}\text { University of } \\
\text { Wisconsin }\end{array}$ & birnessite & & $\begin{array}{l}\text { Initially offered to supply } \\
\text { gram quantities }\end{array}$ & $\begin{array}{l}\text { Joel Pedersen } \\
608-263-4971\end{array}$ & joelpedersen@wisc.edu & \\
\hline $\begin{array}{l}\text { The Ohio State } \\
\text { University }\end{array}$ & birnessite & & Is sending $20-25 \mathrm{~g}$. & $\begin{array}{l}\text { Nicholas Basta } \\
614-292-6282\end{array}$ & basta.4@osu.edu & $\begin{array}{l}\text { Professor of Soil and } \\
\text { Environmental } \\
\text { Chemistry, School of } \\
\text { Environment and Natural } \\
\text { Resources, 410C } \\
\text { Kottman Hall, The Ohio } \\
\text { State University } \\
\text { Columbus, Ohio } 43210\end{array}$ \\
\hline Chevron & all & & $\begin{array}{l}\text { Does not know of } \\
\text { commercial deposit, } \\
\text { samples available for } \\
\text { purchase. Chevron noted } \\
\text { that we will not find these } \\
\text { samples in pure form. }\end{array}$ & Eric Daniels & ericdaniels@chevron.com & \\
\hline $\begin{array}{l}\text { Specialty } \\
\text { Vermiculite } \\
\text { Corp. }\end{array}$ & vermiculite & $\$ 120$ per ton & $\begin{array}{l}\text { Sent } 50 \text { lb sample to } \\
\text { Menomonee Wisc. for } \\
\text { demo. }\end{array}$ & Deb Sobel & dsobel@spvcorp.com & $\begin{array}{l}\text { SPV Corp. } \\
2666 \text { Gaynor Ave. NW } \\
\text { Grand Rapids, MI } 49544\end{array}$ \\
\hline
\end{tabular}




\begin{tabular}{|c|c|c|c|c|c|c|}
\hline Company & Product & Amount/Cost & Comment & Contact & Link & \\
\hline $\begin{array}{l}\text { U.S. Geological } \\
\text { Survey }\end{array}$ & all & & $\begin{array}{l}\text { Sent e-mail with contact } \\
\text { information. Said we should } \\
\text { contact Christy Minerals for } \\
\text { clinoptilolite (below). }\end{array}$ & $\begin{array}{l}\text { Robert Virta } \\
703-648-7726\end{array}$ & rvirta@usgs.gov & $\begin{array}{l}\text { Mineral Commodity } \\
\text { Specialist } \\
\text { National Minerals } \\
\text { Information Center } \\
\text { U.S. Geological Survey } \\
\text { Reston, VA } 20192\end{array}$ \\
\hline $\begin{array}{l}\text { Christy } \\
\text { Minerals } \\
\text { (referred by } \\
\text { Robert Virta } \\
\text { USGS) }\end{array}$ & illite & & $\begin{array}{l}\text { Sent } 2 \text { illitic samples that } \\
\text { formed a mud-like slurry } \\
\text { and were deemed unusable }\end{array}$ & $\begin{array}{l}\text { Jeff Porter } \\
636-585-2214 \\
\# 239\end{array}$ & jporter@christyminerals.com & \\
\hline Chevron & all & & $\begin{array}{l}\text { Pure illite is not easy to } \\
\text { obtain. Birnessite, a } \\
\text { manganese oxide that can } \\
\text { be made synthetically, and } \\
\text { clinoptilolite (a zeolite) are } \\
\text { possibly available } \\
\text { elsewhere. Purity is often } \\
\text { not high. }\end{array}$ & Douglas McCarty & DMcCarty@chevron.com & $\begin{array}{l}\text { Chevron ETC } \\
\text { 3901 Briarpak } \\
\text { Houston, TX } 77042\end{array}$ \\
\hline
\end{tabular}


APPENDIX C SUPPLIERS OF CLINOPTILOLITE AND CHABAZITE

\begin{tabular}{|c|c|c|c|c|c|c|c|c|c|c|c|}
\hline Sample & Company & State & Product Type & Zeolite Type & $\begin{array}{c}\text { Calculated } \\
\text { CEC* }\end{array}$ & $\begin{array}{c}\text { Company } \\
\text { Reported } \\
\text { CEC }\end{array}$ & $\begin{array}{c}\text { Montmoril- } \\
\text { lonite }\end{array}$ & $\begin{array}{l}\text { Opal } \\
\text { C-T }\end{array}$ & Calcite & Quartz & Feldspars \\
\hline R01-0134 & $\begin{array}{l}\text { St. Cloud Mining, } \\
\text { Inc. }\end{array}$ & NM & $\begin{array}{l}\text { Zeo. } \\
\text { (var. Clino.) } \\
6 \times 14 \text { mesh }\end{array}$ & Clinoptilolite & $>120$ & $80-120$ & & & & $3-10 \%$ & Trace \\
\hline R01-0137 & $\begin{array}{l}\text { American Abs. } \\
\text { Products (closed?) }\end{array}$ & OR & Zeolite $6 \times 14$ & Clinoptilolite & $>120$ & & & & & & \\
\hline R01-0138 & Moltan Co L.P. & NV & $\begin{array}{l}\text { Zeolite / } \\
\text { Diatomaceous } \\
\text { Earth }\end{array}$ & $\begin{array}{l}\text { Clinoptilolite/ } \\
\text { Mordenite }\end{array}$ & $50-80$ & & $10-30 \%$ & $3-10 \%$ & & & Trace \\
\hline \multirow[t]{7}{*}{ R01-0287 } & $\begin{array}{l}\text { Ash Meadows } \\
\text { (ZEOX Corp) }\end{array}$ & $\mathrm{CA}$ & Clinoptilolite & Clinoptilolite & $>120$ & & & & & Trace & \\
\hline & KMI Zeolite Inc. & CA & & Clinoptilolite & $>160$ & $160-210$ & & & & $<5 \%$ & \\
\hline & Bear River Zeolite & ID & & Clinoptilolite & 150 & $140-165$ & & $15 \%$ & & & \\
\hline & $\begin{array}{l}\text { Steelhead } \\
\text { Specialty Minerals }\end{array}$ & ID & & Clinoptilolite & & & & & & & \\
\hline & $\begin{array}{l}\text { Teague Mineral } \\
\text { Products }\end{array}$ & $\begin{array}{l}\text { ID / } \\
\text { OR }\end{array}$ & & Clinoptilolite & & & & & & & \\
\hline & $\begin{array}{l}\text { GSA Resources } \\
\text { (ZEOX Corp) }\end{array}$ & $\mathrm{AZ}$ & & Chabazite & & 250 & & & & & \\
\hline & UOP LLC & $\mathrm{AZ}$ & & $\begin{array}{l}\text { Chabazite/ } \\
\text { Mordenite }\end{array}$ & 210 & & & & & & \\
\hline
\end{tabular}




\begin{tabular}{|c|c|c|c|c|c|c|c|c|c|c|c|}
\hline Sample & Company & State & Product Type & Zeolite Type & $\begin{array}{c}\text { Calculated } \\
\text { CEC }^{*}\end{array}$ & $\begin{array}{c}\text { Company } \\
\text { Reported } \\
\text { CEC } \\
\end{array}$ & $\begin{array}{l}\text { Montmoril- } \\
\text { lonite }\end{array}$ & $\begin{array}{l}\text { Opal } \\
\text { C-T }\end{array}$ & Calcite & Quartz & Feldspars \\
\hline \multirow[t]{2}{*}{ R01-0139 } & Zeotech Corp. & $\mathrm{TX}$ & Kitty Crystals & Clinoptilolite & $50-120$ & & $10-30 \%$ & $3-10 \%$ & Trace & & \\
\hline & $\begin{array}{l}\text { Daleco Resources } \\
\text { Corp }\end{array}$ & $\mathrm{TX}$ & & Clinoptilolite & & & & & & & \\
\hline
\end{tabular}

\begin{tabular}{llll}
\multicolumn{1}{c}{ Type } & CEC (Theoretical) & CEC (Practical) & Pore Diameter \\
\hline & & & \\
Clinoptilolite & $230 \mathrm{meq} / 100 \mathrm{~g}$ & $180 \mathrm{meq} / 100 \mathrm{~g}$ & $3.9-5.4$ Angstroms \\
Mordenite & $220 \mathrm{meq} / 100 \mathrm{~g}$ & $175 \mathrm{meq} / 100 \mathrm{~g}$ & $2.9-5.7$ Angstroms \\
Chabazite & $390 \mathrm{meq} / 100 \mathrm{~g}$ & $310 \mathrm{meq} / 100 \mathrm{~g}$ & $3.7-4.2$ Angstroms \\
\hline
\end{tabular}

\footnotetext{
* Implied CEC based on estimated percentage of zeolite present.
} 


\title{
APPENDIX D PRICE FOR VERMICULITE IN BULK
}

\author{
$\mathbb{P} /$ comenom \\ Specialty Vermiculite Corp. \\ Enoree List Pricing \\ Effective thru April 30, 2012 \\ (whereupon pricing will then be reviewed)
}

For information, please contact Customer Service at (800) 342-2017 between the hours of 8:30am and 5:00pm EST.

\section{VCX Vermiculite Ore Concentrate}

\section{Products}

Bulk Shipments:

VCX 205 Bulk

\section{Packaged Goods:}

VCX 205 50\# Bags (60/Pallet)

VCX 205 3000\# Tote

$\begin{array}{lll}\text { Unit Of } & \text { Price Per } & \text { Price Per } \\ \text { Sale } & \underline{\text { Unit }} & \text { Ton }\end{array}$

Ton

$\$ 120.00$

$\$ 120.00$

Bag \$3.84

$\$ 154.00$

Tote

$\$ 212.00$

$\$ 141.00$

Terms and Conditions of Sale:

1. All sales are subject to SPV's Standard Conditions of Sale (see reverse side).

2. Payment Terms: Net 30 Days.

3. All pricing is FOB SPV's plant in Enoree, SC.

Notes:

1. Minimum order is 60 bags or 1 pallet.

2. For additional information on ordering, packaging or delivery, please contact the SPV Customer Service Center or your sales representative.

3. The palletizing configurations included with this pricing are: 50\# Bags: 60 Bags stretch wrapped on a 40 " X 48 " wood pallet. 3000\# Tote With Pallet: 1 Tote on a 48" X 48" wood pallet.

4. Typical volumes per load are:

Description:

Bulk Rail Shipments:

Bulk (LCL) Shipments:

50\# Bags

$3000 \#$ Tote

Unit Of
Sale
Ton
Ton
Bag
Tote

\begin{tabular}{c}
$\begin{array}{c}\text { Unit Volume } \\
\text { Per Shipment }\end{array}$ \\
\hline 95 \\
21 \\
840 \\
14
\end{tabular}

5. Tote Charge \& Return Policy: SPV will provide a returnable tote sack as packaging for this product. A tote sack charge of $\$ 25.00$ each and $\$ 12.00$ each per pallet will be charged on your invoice. This deposit will be credited back to your account once the totes and pallets are returned freight prepaid to the SPV plant that originally shipped the product i.e., Specialty Vermiculite Corp., Hwy 221, Enoree, SC 29335. The totes are to be folded, stacked and banded on a pallet upon return to facilitate counting. Naturally, SPV reserves the right to inspect the returned pallet and totes prior to issuing the credit, with only totes that are returned clean, folded, and in reusable condition being eligible for credit. Deposits on damaged totes/pallets or totes not originally supplied by SPV will not be refunded.

6. A "Block and Brace" charge of $\$ 40.00$ will be added for intermodal shipments.

7. For disposable tote and pallet, add $\$ 20$ per unit (\$13 for tote and $\$ 7$ for pallet). 


\section{REFERENCES}

[1] Kaminski, M., Argonne National Laboratory, private communication, March 2016.

[2] Kaminski, M., Argonne National Laboratory, private communication, March 2016.

[3] Kaminski, M., Argonne National Laboratory, private communication, March 2016.

[4] Kandil, A.E.H., E.A. Saad, A.A.A. Azia, and A.E. Aboelhasan, 2012, Study on adsorption behavior and separation efficiency of naturally occurring clay for some elements by batch experiments, European J. Chem. 3(1), 99-105.

[5] Lemieux, P., J. Wood, C. Hayes, M. Rodgers, D. Schultheisz, T. Peake, and M. Ierardi, 2010, A first-order estimate of debris and waste resulting from a hypothetical radiological dispersal device incident, WM2010 Conference Paper, March 7-11, Phoenix, AZ.

[6] Boe, T., P. Lemieux, D. Schulthiesz, T. Peake, and C. Hayes, 2013, A Planning Tool for Estimating Waste Generated by a Radiological Incident and Subsequent Decontamination Efforts, WM2013 Conference Paper, Feb. 24-March 28, Phoenix, AZ.

[6] Aquinos, M. United State Environmental Protection Agency, private communication, August 2016.

[8] Hamilton, R.L., 1967, Water vapor permeability of polyethylene and other plastic materials, The Bell System Technical Journal, 391-415, February. 



\section{Argonne}

Nuclear Engineering Division

Argonne National Laboratory

9700 South Cass Avenue, Bldg. 208

Argonne, IL 60439-4854

www.anl.gov 